\title{
Restrictions of Steiner Bundles and Divisors on the Hilbert Scheme of Points in the Plane
}

\section{Citation}

Huizenga, Jack. 2012. Restrictions of Steiner Bundles and Divisors on the Hilbert Scheme of Points in the Plane. Doctoral dissertation, Harvard University.

\section{Permanent link}

http://nrs.harvard.edu/urn-3:HUL.InstRepos:9571108

\section{Terms of Use}

This article was downloaded from Harvard University's DASH repository, and is made available under the terms and conditions applicable to Other Posted Material, as set forth at http:// nrs.harvard.edu/urn-3:HUL.InstRepos:dash.current.terms-of-use\#LAA

\section{Share Your Story}

The Harvard community has made this article openly available.

Please share how this access benefits you. Submit a story.

Accessibility 
(C)2012 - Jack W. Huizenga

All rights reserved. 
Thesis advisor

Author

Joseph D. Harris

Jack W. Huizenga

\title{
Restrictions of Steiner bundles and divisors on the Hilbert scheme of points in the plane
}

\begin{abstract}
The Hilbert scheme of $n$ points in the projective plane parameterizes degree $n$ zerodimensional subschemes of the projective plane. We examine the dual cones of effective divisors and moving curves on the Hilbert scheme. By studying interpolation, restriction, and stability properties of certain vector bundles on the plane we fully determine these cones for just over three fourths of all values of $n$.

A general Steiner bundle on $\mathbb{P}^{N}$ is a vector bundle $E$ admitting a resolution of the form

$$
0 \rightarrow \mathcal{O}_{\mathbb{P}^{N}}(-1)^{s} \stackrel{M}{\rightarrow} \mathcal{O}_{\mathbb{P}^{N}}^{s+r} \rightarrow E \rightarrow 0
$$

where the map $M$ is general. We complete the classification of slopes of semistable Steiner bundles on $\mathbb{P}^{N}$ by showing every admissible slope is realized by a bundle which restricts to a balanced bundle on a rational curve. The proof involves a basic question about multiplication of polynomials on $\mathbb{P}^{1}$ which is interesting in its own right.
\end{abstract}




\section{Contents}

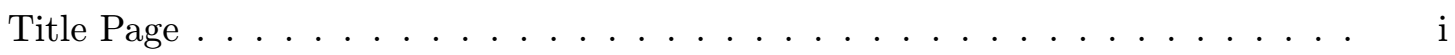

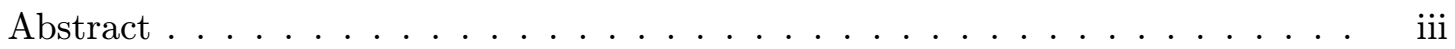

Table of Contents . . . . . . . . . . . . . . . . . . iv

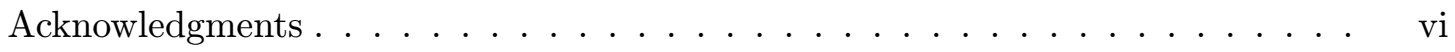

Dedication . . . . . . . . . . . . . . . . vii

1 Introduction 1



1.2 Divisors on $\mathscr{H}_{n}$ and some small examples $\ldots \ldots \ldots \ldots \ldots \ldots$

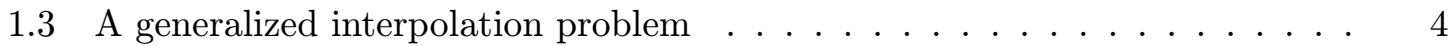

1.4 Semistability of Steiner bundles on $\mathbb{P}^{N} \ldots \ldots \ldots \ldots \ldots \ldots$

1.5 A semistability restriction theorem . . . . . . . . . . . . 8

1.6 Multiplication of polynomials on $\mathbb{P}^{1} \ldots \ldots \ldots \ldots \ldots \ldots \ldots$

1.7 Effective divisors on $\mathscr{H}_{n} \ldots \ldots \ldots \ldots \ldots \ldots \ldots \ldots$

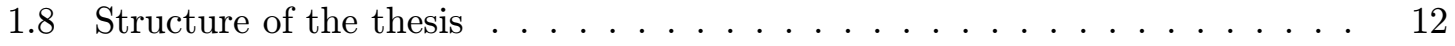

2 Steiner bundles 14

2.1 Multiplication of polynomials on $\mathbb{P}^{1} \ldots \ldots \ldots \ldots \ldots \ldots \ldots$

2.2 Matrices with entries in a fixed series . . . . . . . . . . . . . . 23

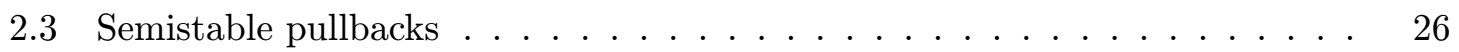

2.4 Slopes of semistable Steiner bundles $\ldots \ldots \ldots \ldots \ldots \ldots$

3 Basic geometry of the Hilbert scheme of points in the plane 31

3.1 Divisors and curves on $\mathscr{H}_{n} \ldots \ldots \ldots \ldots \ldots \ldots \ldots \ldots$

3.2 General results on the effective cone $\ldots \ldots \ldots \ldots \ldots \ldots \ldots$

3.3 The local geometry of $\Delta \ldots \ldots \ldots \ldots \ldots \ldots \ldots$

4 Effective divisors on the Hilbert scheme of points in the plane 43

4.1 Interpolation for bundles on $\mathbb{P}^{2} \ldots \ldots \ldots \ldots \ldots \ldots \ldots$

4.2 Effective divisors on $\mathscr{H}_{n} \ldots \ldots \ldots \ldots \ldots \ldots \ldots$ 
5 The remaining effective cones $\quad 51$

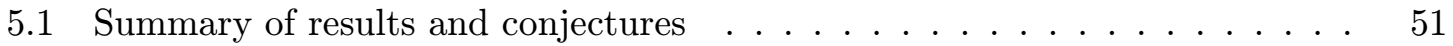

5.2 Existence of secant planes to curves . . . . . . . . . . . . . 56

5.3 Some better moving curves on $\mathscr{H}_{n} \ldots \ldots \ldots \ldots \ldots$

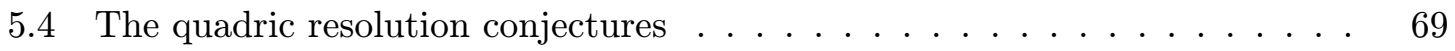

5.5 A minimality property of Steiner bundles . . . . . . . . . 75

A Table of cones of effective divisors and moving curves on $\mathscr{H}_{n} \quad 86$

$\begin{array}{ll}\text { Bibliography } & 93\end{array}$ 


\section{Acknowledgments}

First and foremost, I would like to thank my advisor Joe Harris for his incredible support over the past several years. His endless patience and help is much appreciated. In addition to helping me learn algebraic geometry and develop intuition for the subject, he has helped immensely with navigating graduate school, teaching, and professional development in general. Joe was much more than just a research advisor, and is a true mentor.

I would also like to thank Izzet Coskun for suggesting the thesis problem discussed here, and for many helpful discussions on the topic, as well as for his gracious hospitality during a visit to UIC. Thank you as well to Brendan Hassett, Aaron Bertram, Noam Elkies, and Lawrence Ein, all of whom provided valuable input to this project. 
To my parents 


\section{Chapter 1}

\section{Introduction}

\subsection{Overview}

Throughout mathematics, a particularly important class of spaces are configuration spaces, parameterizing the possible arrangements of various geometric objects. Given a space $X$, one of the simplest types of configuration spaces consists of the collections of $n$ distinct points on $X$; the configuration space for such objects is naturally given by

$$
Y=\left(X^{n} \backslash \Delta\right) / S_{n}
$$

where $\Delta$ is the locus of all $n$-tuples with two equal entries and $S_{n}$ is the symmetric group.

In algebraic geometry, $X$ is usually a projective variety; in particular it is compact. In this case, $Y$ has a major defect, in that it clearly fails to be compact. The obvious solution to this problem is to simply "include" the diagonals in $Y$, leading to the symmetric product $X^{n} / S_{n}$. While this solution works wonderfully when $X$ is a curve, if $X$ has dimension larger than 1 this space will be quite singular. In many ways a better compactification is given by keeping track of the way in which points collide as they become coincident: instead 
of a typical point in the boundary consisting of two copies of one point and $n-2$ other points, the typical point in the boundary should consist of a point together with a tangent direction, together with $n-2$ other points.

Formalizing this idea leads one to define the Hilbert scheme $X^{[n]}$ parameterizing length $n$ zero-dimensional subschemes of $X$. From the viewpoint of algebraic geometry, zerodimensional subschemes are more natural than the zero-dimensional effective cycles parameterized by the symmetric product. Furthermore, in case $X$ is a surface, the Hilbert scheme is a smooth, irreducible projective variety of dimension $2 n[10]$; we note, however, that Hilbert schemes are still very singular in higher dimensions. The problem of describing all possible ways of compactifying $Y$ can be loosely interpreted as the problem of determining all the birational models of $X^{[n]}$. One hopes that these other birational models have descriptions in terms of the geometry of collections of points on $X$. A major theme in the intersection of the study of birational geometry and moduli spaces is to understand the geometric significance of such models.

This problem is a huge undertaking in full generality, so we focus on the case $X=\mathbb{P}^{2}$, and denote by $\mathscr{H}_{n}$ the Hilbert scheme of $n$ points in $\mathbb{P}^{2}$. A project initiated by Bertram and Coskun has been successful in describing the various birational models of $\mathscr{H}_{n}$ for small values of $n$; large values of $n$ are considerably more challenging, however. A first step in this program is to determine the various properties of divisor and curve classes on $\mathscr{H}_{n}$; in particular, we would like to know the cone of effective divisors Eff $\mathscr{H}_{n} \subset \operatorname{Pic} \mathscr{H}_{n}$ on $\mathscr{H}_{n}$-recall that this is the cone spanned by the codimension 1 subvarieties of $\mathscr{H}_{n}$.

The main goal of this thesis is to determine the cone Eff $\mathscr{H}_{n}$ for many values of $n$. It turns out that the problem of constructing divisors spanning this cone is intricately related with many well-known areas of algebraic geometry. In particular, there are connections 
with the stability of vector bundles, generalized interpolation problems on surfaces, and problems regarding multiplication of polynomials on projective spaces.

\subsection{Divisors on $\mathscr{H}_{n}$ and some small examples}

One of the principal reasons for focusing on $\mathbb{P}^{2}$ before other surfaces is that the divisor group Pic $\mathscr{H}_{n}$ is particularly nice. If we fix a line $\ell \subset \mathbb{P}^{2}$, then there is a divisor $H$ given as the locus of length $n$ subschemes $\Gamma \subset \mathbb{P}^{2}$ which meet $\ell$. On the other hand, the locus of nonreduced schemes, i.e. those schemes supported at fewer than $n$ points, forms a divisor $\Delta$. It follows from Fogarty [11] that

$$
\operatorname{Pic} \mathscr{H}_{n}=\mathbb{Z} H \oplus \mathbb{Z}(\Delta / 2) \quad(n \geq 2) .
$$

Since $\mathscr{H}_{1}=\mathbb{P}^{2}$, we will omit the assumption $n \geq 2$ in the future when discussing $\mathscr{H}_{n}$. The class $\Delta$ always spans a boundary ray of Eff $\mathscr{H}_{n}$, so our main question is to determine a generator for the other edge $a H-(b / 2) \Delta$ of this cone. If we call the number $a / b$ the slope of the class $a H-(b / 2) \Delta$, then we are trying to determine the minimum slope of an effective divisor on $\mathscr{H}_{n}$. We note that this cone is in fact closed, as $\mathscr{H}_{n}$ is log Fano and hence a Mori dream space.

Of course the hope is that this other edge corresponds to some geometric property of a collection of points in $\mathbb{P}^{2}$. Some examples are probably in order. In case $n=6$, the extremal divisor is given as the locus of 6 points which lie on a conic. For $n=7$, the divisor is the locus of 7 points such that some 6 of them lie on a conic. The divisor in case $n=8$ is slightly more complicated. Here, 8 points generally lie on a pair of cubics. The pair of cubics meet in a 9th point, and the extremal divisor is the locus where this 9th point lies on a fixed line. In each of these cases, there is a curve class which sweeps out $\mathscr{H}_{n}$ and is 
dual to the divisor; the curve class is given by allowing $n$ points to move in a linear pencil on a smooth curve of some appropriate degree.

The first case where such simple constructions fail to produce the edge of the effective cone is the case $n=12$. A general collection $\Gamma$ of 12 points lies on 3 independent quartics and 9 independent quintics. Furthermore, the quartics vanishing on 12 general points generate the quintics vanishing on those points, in the sense that the multiplication map

$$
H^{0}\left(\mathscr{I}_{\Gamma}(4)\right) \otimes H^{0}\left(\mathcal{O}_{\mathbb{P}^{2}}(1)\right) \rightarrow H^{0}\left(\mathscr{I}_{\Gamma}(5)\right)
$$

is an isomorphism. The locus of $\Gamma$ where this map fails to be an isomorphism forms a divisor spanning the edge of the effective cone of $\mathscr{H}_{12}$. The key point, however, is that we can describe this divisor in a different way. If we define a vector bundle $E$ by the sequence

$$
0 \rightarrow E \rightarrow \mathcal{O}_{\mathbb{P}^{2}}(4)^{3} \stackrel{M}{\rightarrow} \mathcal{O}_{\mathbb{P}^{2}}(5) \rightarrow 0
$$

where the map $M$ is given by a general matrix of linear forms, then this divisor is just given by

$$
D_{E}=\left\{\Gamma \in \mathscr{H}_{12}: H^{0}\left(E \otimes \mathscr{I}_{\Gamma}\right) \neq 0\right\}
$$

In this case, the bundle $E$ is simply $T_{\mathbb{P}^{2}}(2)$.

This last construction provides the correct framework for generalization. We will see that for many values of $n$, there is a vector bundle $E$ such that the extremal effective divisor on $\mathscr{H}_{n}$ can be described as a locus where "interpolation fails" for sections of $E$.

\subsection{A generalized interpolation problem}

A classical problem in algebraic geometry is to determine when polynomial functions can attain prescribed values at prescribed points. In its simplest form, this encompasses 
the technique of Lagrange interpolation, which essentially completely solves the problem over $\mathbb{P}^{1}$ : given any subscheme $\Gamma \subset \mathbb{P}^{1}$ of length $n$ and a prescribed element $\sigma \in H^{0}\left(\mathcal{O}_{\Gamma}(d)\right)$, there is guaranteed to be a degree $d$ homogeneous polynomial $f$ restricting to $\sigma$ whenever $n \leq d+1$, i.e. so long as the space of forms of degree $d$ has dimension at least as big as $h^{0}\left(\mathcal{O}_{\Gamma}(d)\right)$

In higher dimensions, things get much more difficult, so it is typical to focus on general collections of points. A first result is that if $L$ is a line bundle on a variety $X$, then vanishing at a general collection of $n$ points imposes the expected number $\min \left\{n, h^{0}(L)\right\}$ of conditions on sections of $L$; thus interpolation problems are not particularly interesting for general simple points imposing conditions on sections of a line bundle. Sometimes one says line bundles have good postulation for general simple points.

For our purposes, it is important to consider the conditions imposed by simple points on sections of a vector bundle. If $E$ is a rank $r$ vector bundle on $X$, then we expect that vanishing at a general collection of $n$ points imposes $\min \left\{r n, h^{0}(E)\right\}$ conditions on sections of $E$. While this was always true in the line bundle case, it is not at all automatic for vector bundles. For instance, consider the vector bundle

$$
E=\mathcal{O}_{\mathbb{P}^{1}}(-1) \oplus \mathcal{O}_{\mathbb{P}^{1}}(1)
$$

a simple point only imposes a single condition on sections of $E$, where we'd expect it to impose two.

Definition 1.1. A nonzero vector bundle $E$ of rank $r$ on a variety $X$ has interpolation for $n$ points if

1. it has $h^{0}(E)=r n$, and 
2. for general $\Gamma \in X^{[n]}$, we have $H^{0}\left(E \otimes \mathscr{I}_{\Gamma}\right)=0$.

The hypotheses of the definition imply that the restriction map

$$
H^{0}(E) \rightarrow H^{0}\left(\left.E\right|_{\Gamma}\right)
$$

is an isomorphism for general $\Gamma \in \mathscr{H}_{n}$, so perhaps better terminology would be that $E$ has unique interpolation for $n$ points; in the interest of brevity we'll omit this adjective. We may also simply say $E$ has interpolation if the number $n$ of points is clear.

We are interested in this definition because if $X$ is a curve or surface and the vector bundle $E$ on $X$ has interpolation for $n$ points then the locus

$$
D_{E}=\left\{\Gamma \in X^{[n]}: H^{0}\left(E \otimes \mathscr{I}_{\Gamma}\right) \neq 0\right\}
$$

forms a divisor on $X^{[n]}$. In the case of the Hilbert scheme $\mathscr{H}_{n}$ of points in the plane, we can compute this divisor's class as

$$
\left[D_{E}\right]=c_{1}(E) H-\frac{r}{2} \Delta
$$

Since we are attempting to compute only the cone Eff $\mathscr{H}_{n}$, it is worth pointing out that this divisor class is a multiple of the class

$$
\mu(E) H-\frac{1}{2} \Delta
$$

where $\mu(E)=c_{1}(E) / \operatorname{rk}(E)$ is the slope of $E$. If we believe that the effective divisors on $\mathscr{H}_{n}$ should come from vector bundles in this fashion, then computation of the effective cone boils down to the following question.

Question 1.2. What is the minimum slope of a vector bundle $E$ on $\mathbb{P}^{2}$ satisfying interpolation for $n$ points?

We will construct bundles answering this question for many values of $n$, and see that they in fact give divisors spanning the edge of the effective cone. 


\subsection{Semistability of Steiner bundles on $\mathbb{P}^{N}$}

A general Steiner bundle $E$ on $\mathbb{P}^{N}$ of rank $r$ is a vector bundle admitting a resolution of the form

$$
0 \rightarrow \mathcal{O}_{\mathbb{P}^{N}}(-1)^{s} \stackrel{M}{\rightarrow} \mathcal{O}_{\mathbb{P}^{N}}^{s+r} \rightarrow E \rightarrow 0
$$

where $M$ is a general matrix of linear forms. In order for $E$ to be locally free, it is necessary and sufficient that either $s=0$ or $r \geq N$. Classically, some authors only call these bundles Steiner bundles in case $r=N$, so one sometimes calls the bundles with $r>N$ higher rank Steiner bundles; many results on Steiner bundles are much easier in the case $r=N$. These are some of the simplest vector bundles on $\mathbb{P}^{N}$, and much is known about them; we refer the reader to Brambilla [5] for an interesting discussion of many of their properties. Recall that a vector bundle $E$ is called semistable if every coherent subsheaf $F \subset E$ has $\mu(F) \leq \mu(E)$. Observe that the slope of the bundle $E$ given by the above resolution is $\mu(E)=s / r$. The next result classifies the slopes of semistable Steiner bundles.

Theorem 1.3. Define a function $\rho_{N}$ by

$$
\rho_{N}(x)=\frac{1}{N-1+\frac{1}{1+x}}
$$

and put

$$
\phi_{N}=\lim _{i \rightarrow \infty} \rho_{N}^{i}(0)=\frac{\sqrt{N^{2}+2 N-3}}{2 N-2}-\frac{1}{2},
$$

where $\rho^{i+1}=\rho \circ \rho^{i}$ and $\rho^{0}=\mathrm{id}$. Define a set $\Phi_{N}$ by

$$
\Phi_{N}=\left\{\alpha: \alpha>\phi_{N}\right\} \cup\left\{\rho_{N}^{i}(0): i \geq 0\right\} \subset \mathbb{Q}
$$

The set $\Phi_{N}$ consists of all numbers larger than $\phi_{N}$, together with 0 and all the convergents in the continued fraction expansion of $\phi_{N}$ (half of which are larger than $\phi_{N}$ ).

There exists a semistable Steiner bundle of slope $\mu$ on $\mathbb{P}^{N}$ if and only if $\mu \in \Phi_{N}$. 
We call the numbers $\rho_{N}^{i}(0)$ the exceptional slopes of semistable Steiner bundles on $\mathbb{P}^{N}$. We note that a large portion of the proof of the theorem follows from earlier work of Brambilla [4], [5]. In particular, Brambilla's work can be seen to imply the nonexistence of semistable Steiner bundles on $\mathbb{P}^{N}$ with $\mu \notin \Phi_{N}$, and it also shows the existence of semistable Steiner bundles with slope $\mu$ whenever $\mu$ is exceptional. We will show that every slope $\mu \in \Phi_{N}$ can be realized by a semistable Steiner bundle on $\mathbb{P}^{N}$.

Example 1.4. The set $\Phi_{2}$ is particularly important since it is directly relevant to our result below on the Hilbert scheme of points in $\mathbb{P}^{2}$, so we write it down explicitly as

$$
\Phi_{2}=\left\{\alpha: \alpha>\varphi^{-1}\right\} \cup\left\{\frac{0}{1}, \frac{1}{2}, \frac{3}{5}, \frac{8}{13}, \frac{21}{34}, \frac{55}{89}, \cdots\right\} \subset \mathbb{Q} \quad \varphi=\frac{1+\sqrt{5}}{2} .
$$

The exceptional slopes are ratios of consecutive Fibonacci numbers, and they converge to the inverse of the golden ratio.

\subsection{A semistability restriction theorem}

To prove Theorem 1.3 we will actually prove something much stronger. Observe that

if $C \subset \mathbb{P}^{N}$ is a curve and $\left.E\right|_{C}$ is semistable then in fact $E$ is semistable, as a destabilizing subsheaf of $E$ would restrict to a destabilizing subsheaf of $\left.E\right|_{C}$. In the other direction, if $E$ is a semistable vector bundle on $\mathbb{P}^{N}$ and $C$ is a general complete intersection curve of sufficiently high multidegree, then it is known that $\left.E\right|_{C}$ will be semistable; various results to this effect have been given by several authors including Mehta and Ramanathan [15] and Flenner [12]. The general theory does not provide good bounds on how large the degree of $C$ must be, however; furthermore, it also does not usually address what happens for specific types of curves, for instance rational curves. We are able to give the following result. 
Theorem 1.5. Let $E$ be a general Steiner bundle on $\mathbb{P}^{N}$, given by a resolution

$$
0 \rightarrow \mathcal{O}_{\mathbb{P}^{N}}(-1)^{k s} \rightarrow \mathcal{O}_{\mathbb{P}^{N}}^{k(s+r)} \rightarrow E \rightarrow 0
$$

and assume $\mu(E) \in \Phi_{N}$. If $f: \mathbb{P}^{1} \rightarrow \mathbb{P}^{N}$ is a general degree $r$ map and $k$ is sufficiently large, then

$$
f^{*} E \cong \mathcal{O}_{\mathbb{P}^{1}}(s)^{k r}
$$

In particular, $E$ is semistable.

We believe that the theorem should be true for every $k \geq 1$; we are able to prove this only when $\mu(E)$ is exceptional, however. The main idea of the proof is to show that the property that the pullback is balanced corresponds to some general matrix with entries in an $(N+1)$-dimensional series $V \subset H^{0}\left(\mathcal{O}_{\mathbb{P}^{1}}(r)\right)$ giving an isomorphism between two vector spaces of polynomials on $\mathbb{P}^{1}$. We then look at an incidence correspondence consisting of pairs of matrices and vectors in their kernels, and conclude by a dimension count that the general such matrix has no kernel. The key ingredient in the dimension count is discussed in the next section.

\subsection{Multiplication of polynomials on $\mathbb{P}^{1}$}

Consider the following basic problem about polynomial multiplication. Suppose we have an $(N+1)$-dimensional subspace $V$ of the space $S_{r} \subset k[u, v]$ of homogeneous polynomials of degree $r$ in $u, v$. If $W$ is a subspace of $S_{s-1}$, think of $W$ as filling up the "fraction"

$$
\eta(W)=\frac{\operatorname{dim} W}{\operatorname{dim} S_{s-1}}
$$

of $S_{s-1}$ (noting that $\operatorname{dim} S_{s-1}=s$ ). The space $V \cdot W$ spanned by products of elements of $V$ and $W$ lies in $S_{r+s-1}$. 
Question 1.6. Let $V \subset S_{r}$ be a general series of dimension $N+1$, and fix a positive integer s. Is it true that for every $W \subset S_{s-1}$ we have $\eta(V \cdot W) \geq \eta(W)$ ?

In other words, for general $V$, does multiplication of an arbitrary series $W$ by $V$ never decrease the fraction of the ambient space that is occupied? Our proof of Theorem 1.5 works whenever this question has an affirmative answer.

Simple examples show that the answer is not always yes. For instance, if $r / s>N$ and $V \subset S_{r}$ is any $(N+1)$-dimensional series, then the multiplication map

$$
V \otimes S_{s-1} \rightarrow S_{r+s-1}
$$

cannot be surjective. Taking $W=S_{s-1}$, we thus have $\eta(V \cdot W)<\eta(W)=1$.

The technical heart of the thesis lies in our answer to Question 1.6.

Theorem 1.7. Let $V \subset S_{r}$ be a general series of dimension $N+1$, and fix an integer $s$. Assume $r$ and $s$ are coprime. Then every series $W \subset S_{s-1}$ satisfies $\eta(V \cdot W) \geq \eta(W)$ if and only if $s / r \in \Phi_{N}$ (see Theorem 1.3 for the definition of $\Phi_{N}$ ).

The coprimality assumption is mainly technical, and we suspect it is unnecessary; we will in fact prove the more interesting reverse direction without this assumption. The proof of the difficult direction essentially constructs a series $V$ with the required properties under the assumption $s / r \in \Phi_{N}$. To prove the easier direction, we show that if there is an $(N+1)$ dimensional series $V$ with the required properties for $s, r$, then there is a semistable Steiner bundle on $\mathbb{P}^{N}$ with slope $s / r$. 


\subsection{Effective divisors on $\mathscr{H}_{n}$}

Our initial interest in the restriction result (Theorem 1.5) came from the observation that it allows us to show that certain twists and/or duals of general Steiner bundles on $\mathbb{P}^{2}$ satisfy interpolation for $n$ points. In particular, by specializing $n$ points to certain points on rational curves, we find that $H^{0}\left(E \otimes \mathscr{I}_{\Gamma}\right)=0$ for certain vector bundles $E$ with $h^{0}(E)=r n$, proving the difficult part of interpolation.

Once we've constructed effective divisors on $\mathscr{H}_{n}$, we must show they are extremal. Given an effective divisor $D$, we can show it is extremal by finding a dual moving curve class, i.e. a curve class $\gamma \in N_{1}\left(\mathscr{H}_{n}\right)$ such that $\gamma \cdot D=0$ and irreducible representatives of $\gamma$ sweep out a dense open subset of $\mathscr{H}_{n}$. In this manner, we construct the nontrivial edge of Eff $\mathscr{H}_{n}$ for roughly $76 \%$ of all values of $n$. Our new results on Eff $\mathscr{H}_{n}$ are summarized in the next theorem. Previously, the cone was only known for sporadic values of $n$ with zero density in the natural numbers.

Theorem 1.8. Write

$$
n=\frac{r(r+1)}{2}+s \quad(0 \leq s \leq r)
$$

there is a unique such decomposition.

1. If $s / r \in \Phi_{2}$ (see Example 1.4), then the divisor

$$
D=\left(r^{2}-r+s\right) H-\frac{r}{2} \Delta
$$

spans an edge of the effective cone of $\mathscr{H}_{n}$; sufficiently large multiples of this class are the loci where interpolation fails for a general vector bundle $E$ with resolution

$$
0 \rightarrow \mathcal{O}_{\mathbb{P}^{2}}(r-2)^{k s} \rightarrow \mathcal{O}_{\mathbb{P}^{2}}(r-1)^{k(s+r)} \rightarrow E \rightarrow 0
$$


These divisors are dual to moving curves $\gamma$ on $\mathscr{H}_{n}$ given by allowing $n$ points to move in a linear pencil on a smooth curve of degree $r$.

2. If $1-\frac{s+1}{r+2} \in \Phi_{2}$ and $s \geq 1$, then the divisor

$$
D^{\prime}=\left(r^{2}+r+s-1\right) H-\frac{r+2}{2} \Delta
$$

spans an edge of the effective cone of $\mathscr{H}_{n}$; sufficiently large multiples of this class are the loci where interpolation fails for a general vector bundle $F$ with resolution

$$
0 \rightarrow F \rightarrow \mathcal{O}_{\mathbb{P}^{2}}(r)^{k(2 r-s+3)} \rightarrow \mathcal{O}_{\mathbb{P}^{2}}(r+1)^{k(r-s+1)} \rightarrow 0 .
$$

These divisors are dual to moving curves $\gamma^{\prime}$ on $\mathscr{H}_{n}$ given by allowing $n$ points to move in a linear pencil on a smooth curve of degree $r+2$.

The cases where the effective cone is not determined by the theorem are those $n$ such that the ratio $s / r$ is close to (but not equal to) $1 / 2$.

\subsection{Structure of the thesis}

We begin the thesis by proving our results on Steiner bundles, including the polynomial multiplication result, in Chapter 2. In Chapter 3 we take some time to verify basic properties of the Hilbert scheme $\mathscr{H}_{n}$, most of which are known but have not been collected into a good single source. This includes the computation of the Picard group (at least over $\mathbb{Q}$ ), verification of the fact that the Hilbert scheme is a Mori dream space (so that the effective cone is closed), and a local study of the boundary divisor $\Delta$. We then apply our results on Steiner bundles to prove our main theorem on Hilbert schemes in Chapter 4. In Chapter 5 we discuss progress on determining the effective cone of $\mathscr{H}_{n}$ for values of $n$ not covered 
by our main theorem. This includes a construction of more highly sloped moving curves for many values of $n$, as well as a series of conjectures predicting the cone for many $n$. As there are many results of many flavors in this chapter, we refer the reader to Section 5.1 for a summary of the current state of affairs.

Finally, at the end of the thesis Appendix A provides a table describing the cone of effective divisors and dual cone of moving curves for small $n$. 


\section{Chapter 2}

\section{Steiner bundles}

In this chapter, we will prove our main results on Steiner bundles on $\mathbb{P}^{N}$. The majority of the work lies in proving Theorem 1.7 regarding polynomial multiplication on $\mathbb{P}^{1}$, and we do this in Section 2.1. We then use this theorem to prove a result about matrices with polynomial entries lying in a fixed linear series on $\mathbb{P}^{1}$ in Section 2.2. This result allows us to show that pullbacks of Steiner bundles to general rational curves of appropriate degree are balanced, which we show in the next section. Finally, we conclude the chapter by completing the classification of slopes of semistable Steiner bundles and by proving converses of all the earlier results in the chapter.

\subsection{Multiplication of polynomials on $\mathbb{P}^{1}$}

Our first goal is to prove Theorem 1.7. For actually proving the theorem, a renormalization of our notation will be useful. Recall that we write $S_{a}=H^{0}\left(\mathcal{O}_{\mathbb{P}^{1}}(a)\right)$; we choose an affine coordinate $u$ on $\mathbb{P}^{1}$, so $S_{a}$ corresponds to polynomials of degree at most $a$ in $u$. To avoid trivialities, we will assume $b>a$ and $N \geq 2$ throughout this section. If $V \subset S_{b-a}$ is 
an $N$-dimensional series and $W \subset S_{a-1}$ is a nonempty series, we define the filling ratio of $W$ with respect to $V$ by

$$
\mu_{V}(W)=\frac{\operatorname{dim}(V \cdot W)}{\operatorname{dim} W}
$$

where $V \cdot W$ denotes the image of $V \otimes W \rightarrow S_{b-1}$. In terms of filling ratios, the theorem aims to classify when $\mu_{V}(W) \geq b / a$ holds for every $W \subset S_{a-1}$ when $V \subset S_{b-a}$ is a general fixed series of dimension $N$.

In the introduction we had $r=b-a$ and $s=a$, so

$$
\frac{b}{a}=1+\left(\frac{s}{r}\right)^{-1}
$$

We thus define a set

$$
\Psi_{N}=1+\Phi_{N-1}^{-1}
$$

defining arithmetic options on sets elementwise, and note that $b / a \in \Psi_{N}$ if and only if $s / r \in \Phi_{N-1}$, where we interpret division by zero as yielding $\infty$. The set $\Psi_{N}$ has a nicer description than $\Phi_{N-1}$ does: if we put

$$
\theta(x)=N-x^{-1}
$$

and $\psi_{N}=\lim _{i \rightarrow \infty} \theta^{i}(\infty)$, where we interpret $\theta(\infty)$ as $N$, then it is trivial to verify

$$
\Psi_{N}=\left\{\alpha: 1<\alpha<\psi_{N}\right\} \cup\left\{\theta^{i}(\infty): i \geq 0\right\} \subset \mathbb{Q} \cup\{\infty\}
$$

We remark that

$$
\psi_{N}=\frac{N+\sqrt{N^{2}-4}}{2}
$$

so $N-1 \leq \psi_{N}<N$. Furthermore, every finite element of $\Psi_{N}$ is no larger than $N$.

Notice that to prove the theorem it suffices to find a single $N$-dimensional $V$ with the required property. The next theorem refines one direction of Theorem 1.7, and its statement will be a bit easier to work with. 
Theorem 2.1. Suppose $b / a \in \Psi_{N}$, and let $V \subset S_{b-a}$ be a general series of dimension $N$. For every nonempty $W \subset S_{a-1}$ we have $\mu_{V}(W) \geq b / a$.

Proof. The proof proceeds in two steps. First, we will show in Proposition 2.3 below that the theorem is true when $1<b / a \leq N-1$ via a direct argument with monomials. The theorem is also vacuously true when $a=0$ so that $b / a=\infty$. Next, if $N-1<b / a \leq N$ and $b / a \in \Psi_{N}$, put $a^{\prime}=N a-b$ and $b^{\prime}=a$. We will show in Lemma 2.4 that proving the theorem for $a, b$, and $N$ can be reduced to proving the theorem for $a^{\prime}, b^{\prime}$, and $N$. Notice that the ratio $b^{\prime} / a^{\prime}$ satisfies

$$
\theta\left(\frac{b^{\prime}}{a^{\prime}}\right)=N-\frac{a^{\prime}}{b^{\prime}}=\frac{b}{a}, \quad \text { so } \quad \frac{b^{\prime}}{a^{\prime}}=\theta^{-1}\left(\frac{b}{a}\right)
$$

Now look at the function

$$
\theta^{-1}(x)=\frac{1}{N-x}
$$

We observe that $\theta^{-1}$ has a fixed point at $\psi_{N}$ (this explains the essential nature of $\psi_{N}$ to the theorem), and that repeated application of $\theta^{-1}$ will eventually decrease any ratio $b / a$ with $N-1<b / a<\psi_{N}$ to a ratio $\theta^{-n}(b / a)$ with $1<\theta^{-n}(b / a) \leq N-1$, where the theorem is already known to hold. On the other hand, if $b / a \in \Psi_{N}$ and $b / a>\psi_{N}$, then $b / a=\theta^{i}(\infty)$ for some $i$, and applying $\theta^{-i}$ reduces us to the trivial case of $b / a=\infty$, completing the proof.

On a first reading, it may make sense to skip to the next section at this point, as what follows is both self-contained and the most technical portion of the thesis. We now proceed to prove the two results cited in the previous proof; we first show that the theorem holds when $1<b / a \leq N-1$. All the difficulty of the result occurs already in case $N=3$, so we focus on this case first. 
Lemma 2.2. Suppose $1<b / a \leq 2$, and let $c$ be the remainder upon division of $a$ by $b-a$.

The net

$$
V=\left\langle 1, u^{c}, u^{b-a}\right\rangle
$$

satisfies $\mu_{V}(W) \geq b / a$ for every nonempty $W \subset S_{a-1}$.

Proof. Let $W \subset S_{a-1}$, and consider the space $W^{\prime} \subset S_{a-1}$ spanned by leading terms (with respect to $u$ ) of polynomials in $W$. Clearly $\operatorname{dim} W=\operatorname{dim} W^{\prime}$. When we multiply a monomial in $V$ by a monomial in $W^{\prime}$, we obtain a monomial which is the leading term of an element of $V \cdot W$. This implies that $\operatorname{dim}\left(V \cdot W^{\prime}\right) \leq \operatorname{dim}(V \cdot W)$, and therefore $\mu_{V}\left(W^{\prime}\right) \leq \mu_{V}(W)$. Thus to prove the result, we may assume $W$ is spanned by monomials.

We now rephrase the question in terms of sumsets. Given a set $S \subset\{0, \ldots, a-1\}$, we define the filling ratio of $S$ by

$$
\mu(S)=\frac{|S+\{0, c, b-a\}|}{|S|}
$$

where a sum $S+T$ of two sets of integers denotes $\{s+t: s \in S, t \in T\}$. We must show $\mu(S) \geq b / a$ for any nonempty $S$.

We first reduce to the case where $a, b$ are coprime. If $k \mid a$ and $k \mid b$ then $k \mid(b-a)$ and $k \mid c$. It is easy to see that if the result holds for $a / k$ and $b / k$ then it holds for $a$ and $b$; one can partition $\{0, \ldots, a-1\}$ into the sets

$$
\{0, k, \ldots, a-k\} \cup\{1, k+1, \ldots, a-k+1\} \cup \cdots \cup\{k-1,2 k-1, \ldots, a-1\},
$$

and addition of $\{0, c, b-a\}$ respects this decomposition.

Now assuming $a$ and $b$ are coprime, first suppose that the natural map $\alpha: S \rightarrow \mathbb{Z} /(b-$ $a) \mathbb{Z}$ is surjective, so that $S$ contains an integer of each residue class mod $b-a$. Then 
$|S+\{0, b-a\}| \geq|S|+b-a$, since $|S+\{0, b-a\}|$ contains a new element in each residue class $\bmod b-a$. But $|S| \leq a$, so we conclude

$$
\mu(S)=\frac{|S+\{0, c, b-a\}|}{|S|} \geq \frac{|S+\{0, b-a\}|}{|S|} \geq 1+\frac{(b-a)}{|S|} \geq \frac{b}{a}
$$

Next assume $\alpha$ is not surjective. Think of $\mathbb{Z} /(b-a) \mathbb{Z}$ as a graph by joining two residues by an edge whenever they differ by $c$. Since $c$ is relatively prime to $b-a$, this graph is a connected cycle on $b-a$ vertices. If the induced subgraph $\alpha(S)$ is not connected, one of its connected components $T \subset \mathbb{Z} /(b-a) \mathbb{Z}$ must satisfy $\mu\left(\alpha^{-1}(T)\right) \leq \mu(S)$. Indeed, if $T$ is a component of $\alpha(S)$ and $\bar{T}$ is the complement of $T$ in $\mathbb{Z} /(b-a) \mathbb{Z}$, then by construction the sets $\alpha^{-1}(T)+\{0, c, b-a\}$ and $\alpha^{-1}(\bar{T})+\{0, c, b-a\}$ are disjoint and have union $S+\{0, c, b-a\}$ so

$$
\mu(S)=\frac{\left|\alpha^{-1}(T)\right|}{|S|} \mu\left(\alpha^{-1}(T)\right)+\frac{\left|\alpha^{-1}(\bar{T})\right|}{|S|} \mu\left(\alpha^{-1}(\bar{T})\right)
$$

is the weighted average of $\mu\left(\alpha^{-1}(T)\right)$ and $\mu\left(\alpha^{-1}(\bar{T})\right)$. Thus at least one of these numbers is no larger than $\mu(S)$. Continuing to break up $\bar{T} \cap \alpha(S)$ into components if necessary, we eventually find a component with the desired property. We may thus assume that $\alpha(S)$ is connected.

Now that $\alpha(S)$ is connected, it must look like an arithmetic progression with step size $c$ :

$$
\alpha(S)=\{d, d+c, d+2 c, \ldots, d+k c\} \quad(\bmod b-a)
$$

where $k$ is between 0 and $b-a-2$; the above listed elements are all distinct. We can approximate

$$
|S+\{0, c, b-a\}| \geq|S|+k+2,
$$

since $S+\{0, b-a\}$ contains at least $k+1$ elements not in $S$ (one in each residue class mod 
$b-a$ in $\alpha(S))$ and $S+c$ has an element whose residue $\bmod b-a$ has class $d+(k+1) c$, which is not a residue of any element of $S+\{0, b-a\}$.

The last ingredient we need to bound the filling ratio of $S$ is an upper bound on its size. If $\beta:\{0, \ldots, a-1\} \rightarrow \mathbb{Z} /(b-a) \mathbb{Z}$ is the residue map, we can say that $|S| \leq\left|\beta^{-1}(\alpha(S))\right|$. We write $a=(b-a) q+c$ as in the division algorithm. The fiber of $\beta$ over a residue $e$ in $\mathbb{Z} /(b-a) \mathbb{Z}$ has size $q$ or $q+1$ : it is $q+1$ if $0 \leq e<c$, and it is $q$ otherwise. We must therefore determine how many $h$ of the residues $e$ in $\alpha(S)$ satisfy $0 \leq e<c$.

Instead of thinking about residues, think about integers. Starting at each multiple of $b-a$ we place a "bucket" $c$ integers wide, and we are asking how many terms in our arithmetic progression with step size $c$ land in the buckets. Since the step size of the progression is the same as the bucket width, each bucket can contain at most one term from the progression, and it is impossible to "skip over" a bucket. The arithmetic progression will therefore hit as many buckets as possible if we have $d=c-1$, so that the progression starts at the rightmost edge of a bucket. The number of buckets hit will equal one more than the number of times the sequence passes a multiple of $b-a$. Therefore

$$
h \leq 1+\frac{c-1+k c}{b-a}<1+(k+1) \frac{c}{b-a} .
$$

We conclude

$$
|S| \leq\left|\beta^{-1}(\alpha(S))\right|=q(k+1)+h<1+(k+1)\left(q+\frac{c}{b-a}\right) .
$$

Finally, we finish the proof by observing

$$
\begin{aligned}
\mu(S) & =\frac{|S+\{0, c, b-a\}|}{|S|} \geq \frac{|S|+k+2}{|S|}=1+\frac{k+2}{|S|} \\
& >1+\frac{(b-a)(k+2)}{(b-a)+(k+1)((b-a) q+c)}=\frac{b k+3 b-2 a}{a k+b} .
\end{aligned}
$$


But

$$
\frac{b k+3 b-2 a}{a k+b} \geq \frac{b}{a}
$$

since cross-multiplying shows that it is equivalent to

$$
(b-a)(2 a-b) \geq 0
$$

which is true by assumption. We conclude $\mu(S)>b / a$, as was to be shown.

The equivalent result for $N>3$ follows readily from the result for $N=3$, as we will now demonstrate.

Proposition 2.3. Theorem 2.1 holds when $1<b / a \leq N-1$.

Proof. Write $b-a=q a+r$, choosing the remainder in the range $0<r \leq a$. Let $V^{\prime} \subset S_{r}$ be a net such that for every $W \subset S_{a-1}$ we have $\mu_{V^{\prime}}(W) \geq(r+a) / a$; this is possible by the lemma since $1<(r+a) / a \leq 2$. Define

$$
V=\left\langle 1, u^{a}, u^{2 a}, \cdots, u^{(q-1) a}\right\rangle+u^{q a} V^{\prime} .
$$

Since

$$
q=\frac{b}{a}-1-\frac{r}{a}<\frac{b}{a}-1 \leq N-2
$$

we find

$$
\operatorname{dim} V \leq q+3<N+1
$$

and therefore $\operatorname{dim} V \leq N$. But for $W \subset S_{a-1}$ we have

$$
V \cdot W \cong W \oplus u^{a} W \oplus u^{2 a} W \oplus \cdots \oplus u^{(q-1) a} W \oplus u^{q a}\left(V^{\prime} \cdot W\right)
$$

since the polynomials in $W$ have degree smaller than $a$. Thus

$$
\operatorname{dim}(V \cdot W)=q \operatorname{dim} W+\operatorname{dim}\left(V^{\prime} \cdot W\right)
$$


and

$$
\mu_{V}(W)=q+\mu_{V^{\prime}}(W) \geq q+1+\frac{r}{a}=\frac{b}{a}
$$

completing the proof.

We now complete the second step of the proof of the theorem.

Lemma 2.4. Put $a^{\prime}=N a-b$ and $b^{\prime}=a$, and assume $N-1<b / a \leq N$. If Theorem 2.1 holds for $a^{\prime}, b^{\prime}, N$, then it holds for $a, b, N$.

Proof. Consider an inclusion of vector bundles

$$
0 \rightarrow \mathcal{O}_{\mathbb{P}^{1}}\left(a^{\prime}-1\right) \oplus \mathcal{O}_{\mathbb{P}^{1}}(-1)^{N-2} \stackrel{M}{\rightarrow} \mathcal{O}_{\mathbb{P}^{1}}(a-1)^{N} \rightarrow Q \rightarrow 0
$$

given by a general matrix $M$ of polynomials, and let $Q$ be the cokernel. Since $N \geq 2$, we find that $Q$ is locally free, hence equals $\mathcal{O}_{\mathbb{P}^{1}}(b-1)$ by a Chern class calculation. We thus have an exact sequence on global sections

$$
0 \rightarrow S_{a^{\prime}-1} \stackrel{\alpha}{\rightarrow} S_{a-1}^{N} \stackrel{\beta}{\rightarrow} S_{b-1} \rightarrow 0
$$

Here the map $\beta$ is specified by elements of an at most $N$-dimensional series $V \subset S_{b-a}$; these polynomials are the $(N-1) \times(N-1)$-minors of the matrix $M$. On the other hand, the map $\alpha: S_{a^{\prime}-1} \rightarrow S_{a-1}^{N}=S_{b^{\prime}-1}^{N}$ is given by independent elements of a general $N$-dimensional series $V^{\prime} \subset S_{b^{\prime}-a^{\prime}}$, so by assumption we may assume $V^{\prime}$ satisfies the conclusion of the theorem for $a^{\prime}, b^{\prime}, N$. We claim $V$ satisfies the conclusion of the theorem for $a, b, N$.

To see this, suppose $W \subset S_{a-1}$ is chosen such that the filling ratio $\mu_{V}(W)$ is minimal. If $\mu_{V}(W)=N$ then we are done, so we may assume $\mu_{V}(W)<N$, which is to say that $\left.\beta\right|_{W^{N}}: W^{N} \rightarrow S_{b-1}$ is not injective. Write $K=\alpha\left(S_{a^{\prime}-1}\right)=\operatorname{ker} \beta$. Then $W^{N} \cap K$ is 
non-empty. Let $W^{\prime} \subset W$ be the subseries spanned by entries of elements of $W^{N} \cap K$. Then by construction

$$
W^{N} \cap K=\left(W^{\prime}\right)^{N} \cap K
$$

For any series $W \subset S_{a-1}$ we have an exact sequence

$$
0 \rightarrow W^{N} \cap K \rightarrow W^{N} \rightarrow V \cdot W \rightarrow 0
$$

so

$$
\operatorname{dim}(V \cdot W)=N \operatorname{dim} W-\operatorname{dim}\left(W^{N} \cap K\right)
$$

and

$$
\mu_{V}(W)=N-\frac{\operatorname{dim}\left(W^{N} \cap K\right)}{\operatorname{dim} W}
$$

Thus

$$
\mu_{V}(W)=N-\frac{\operatorname{dim}\left(W^{N} \cap K\right)}{\operatorname{dim} W} \geq N-\frac{\operatorname{dim}\left(\left(W^{\prime}\right)^{N} \cap K\right)}{\operatorname{dim} W^{\prime}}=\mu_{V}\left(W^{\prime}\right)
$$

with equality if and only if $W=W^{\prime}$. Since $W$ was chosen with minimal filling ratio, $W=W^{\prime}$, i.e. $W$ is spanned by the entries of elements of $W^{N} \cap K$.

Now put $U=\alpha^{-1}\left(W^{N} \cap K\right) \subset S_{a^{\prime}-1}$. Clearly $\operatorname{dim} U=\operatorname{dim}\left(W^{N} \cap K\right)$ since $\alpha$ maps $S_{a^{\prime}-1}$ isomorphically onto $K$. By the previous paragraph, we see that $V^{\prime} \cdot U=W$ since $V^{\prime} \cdot U$ contains all the entries of any element of $W^{N} \cap K$.

Finally, since the result holds for $V^{\prime}$ we have

$$
\begin{aligned}
\frac{1}{N-\frac{b}{a}}=\frac{b^{\prime}}{a^{\prime}} & \leq \mu_{V^{\prime}}(U)=\frac{\operatorname{dim}\left(U \cdot V^{\prime}\right)}{\operatorname{dim} U}=\frac{\operatorname{dim} W}{\operatorname{dim}\left(W^{N} \cap K\right)} \\
& =\frac{\operatorname{dim} W}{N \operatorname{dim} W-\operatorname{dim}(V \cdot W)}=\frac{1}{N-\mu_{V}(W)}
\end{aligned}
$$

and we conclude $\mu_{V}(W) \geq b / a$. 


\subsection{Matrices with entries in a fixed series}

In this section we prove a result which gives the main link between Steiner bundles and our polynomial multiplication question.

Proposition 2.5. Let $V \subset S_{b-a}$ be a general series of dimension $N$, and let $M$ be a general $a k \times b k$ matrix with entries in $V$. Assume $b / a \in \Psi_{N}$. If $k$ is sufficiently large, then the map

$$
S_{a-1}^{b k} \stackrel{M}{\rightarrow} S_{b-1}^{a k}
$$

is an isomorphism.

Proof. We first show that it suffices to consider the case where $a, b$ are coprime. For say $a=a^{\prime} d, b=b^{\prime} d$, with $\left(a^{\prime}, b^{\prime}\right)=1$. We can decompose

$$
S_{a-1} \cong S_{a^{\prime}-1}^{d} \quad S_{b-1} \cong S_{b^{\prime}-1}^{d}
$$

where the $i$ th factor of each decomposition is spanned by all monomials $u^{c}$ with $c \equiv i$ $(\bmod d)$. If we have a series $V^{\prime} \subset S_{b^{\prime}-a^{\prime}}$ which proves the theorem for $a^{\prime}, b^{\prime}$, then we can regard it as a series $V \subset S_{b-a}$ by making the change of variables $u \mapsto u^{d}$. Then a general matrix $M$ with entries in $V$ will respect the decompositions

$$
S_{a-1}^{b k} \cong\left(S_{a^{\prime}-1}^{b k}\right)^{d} \quad S_{b-1}^{a k} \cong\left(S_{b^{\prime}-1}^{a k}\right)^{d}
$$

and give an isomorphism

$$
S_{a^{\prime}-1}^{b k} \stackrel{\cong}{\rightarrow} S_{b^{\prime}-1}^{a k}
$$

on each of the $d$ factors separately. Thus $M$ is an isomorphism.

We now assume $a$ and $b$ are coprime. Choose $V$ so that the conclusion of Theorem 2.1 is satisfied. Observe that $S_{a-1}^{b k}$ and $S_{b-1}^{a k}$ both have dimension $a b k$, so to show some $M$ is 
an isomorphism, it suffices to show it is injective. Consider the incidence correspondence

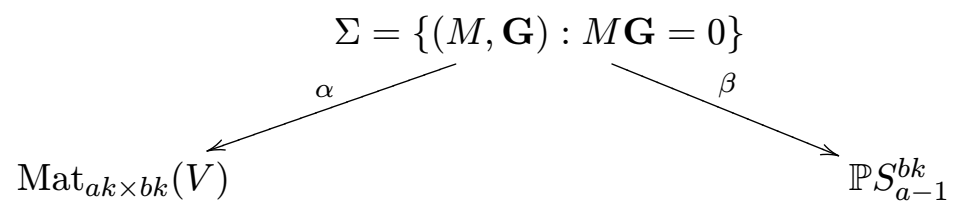

where $\operatorname{Mat}_{a k \times b k}(V)$ denotes the space of $a k \times b k$ matrices with entries in $V$. We would like to prove that

$$
\operatorname{dim} \Sigma<\operatorname{dim} \operatorname{Mat}_{a k \times b k}(V)=N a b k^{2},
$$

since then $\alpha$ is not dominant and the general matrix $M$ gives an isomorphism. We estimate the dimension of $\Sigma$ by looking at the projection $\beta$. For $\mathbf{G} \in \mathbb{P} S_{a-1}^{b k}$, we denote by $W_{\mathbf{G}}$ the subspace of $S_{a-1}$ spanned by the entries of $\mathbf{G}$. We put

$$
X_{\ell}=\left\{\mathbf{G}: \operatorname{dim} W_{\mathbf{G}} \leq \ell\right\} \subset \mathbb{P} S_{a-1}^{b k},
$$

and we easily compute

$$
\operatorname{dim}\left(X_{\ell} \backslash X_{\ell-1}\right)=\operatorname{dim} \operatorname{Gr}\left(\ell, S_{a-1}\right)+b k \ell-1=\ell(a-\ell)+b k \ell-1 .
$$

We decompose

$$
\Sigma=\bigcup_{\ell=1}^{a} \beta^{-1}\left(X_{\ell} \backslash X_{\ell-1}\right),
$$

so we must show each $\beta^{-1}\left(X_{\ell} \backslash X_{\ell-1}\right)$ has dimension smaller than $N a b k^{2}$.

To analyze the dimension of $\beta^{-1}\left(X_{\ell} \backslash X_{\ell-1}\right)$, we must bound the dimension of the fiber over a point $\mathbf{G} \in X_{\ell} \backslash X_{\ell-1}$. If $\mathbf{G}=\left(g_{1}, \ldots, g_{b k}\right)$, then a matrix $M$ satisfies $M \mathbf{G}=0$ exactly when each of its $a k$ rows are in the kernel of

$$
\begin{aligned}
V^{b k} & \rightarrow S_{a-1} \\
\left(f_{1}, \ldots, f_{b k}\right) & \mapsto f_{1} g_{1}+\cdots+f_{b k} g_{b k}
\end{aligned}
$$


The image of this map is $V \cdot W_{\mathbf{G}}$, so the kernel has dimension $N b k-\operatorname{dim}\left(V \cdot W_{\mathbf{G}}\right)$. Thus the fiber of $\beta$ over $\mathbf{G}$ has dimension

$$
\operatorname{dim} \beta^{-1}(\mathbf{G})=\left(N b k-\operatorname{dim}\left(V \cdot W_{\mathbf{G}}\right)\right) a k .
$$

Now if $W \in \operatorname{Gr}\left(\ell, S_{a-1}\right)$ is chosen to minimize $\operatorname{dim}(V \cdot W)$, we estimate

$$
\operatorname{dim} \beta^{-1}\left(X_{\ell} \backslash X_{\ell-1}\right) \leq \ell(a-\ell)+b k \ell-1+N a b k^{2}-a k \operatorname{dim}(V \cdot W) .
$$

We need this quantity to be smaller than $N a b k^{2}$, which amounts to saying

$$
\ell(a-\ell)-1<k(a \operatorname{dim}(V \cdot W)-b \ell) .
$$

If $\ell=a$ then this inequality is immediate since $V \cdot S_{a-1}=S_{b-1}$. Otherwise, if $\ell<a$, we know $\mu_{V}(W) \geq b / a$. This inequality is in fact strict, since

$$
\mu_{V}(W)=\frac{\operatorname{dim}(V \cdot W)}{\operatorname{dim} W}
$$

has denominator smaller than $a$ and $b / a$ is already written in lowest terms. Thus

$$
a \operatorname{dim}(V \cdot W)-b \ell>0
$$

and for $k$ sufficiently large Inequality (2.1) holds.

Remark 2.6. We believe the conclusion of the proposition holds even if $k=1$. The argument given here is not refined enough to prove this, however. To see this, look at Inequality $(2.1)$ in case $k=1$ :

$$
\ell(a-\ell)-1<a \operatorname{dim}(V \cdot W)-b \ell .
$$

This inequality is equivalent to the inequality

$$
\mu_{V}(W) \geq \frac{b}{a}+1-\frac{\operatorname{dim} W}{a} .
$$


However, we observe that this inequality will not be satisfied if $b / a>N-1$ and $\operatorname{dim} W$ is small compared to $a$, since then the inequality would imply $\mu_{V}(W)>N$. This inequality is never satisfied for any $V$ and $W$.

To prove the proposition by this general method for $k=1$, it would be necessary to further stratify the $X_{\ell} \backslash X_{\ell-1}$ into loci of the form

$$
Y_{r, \ell}=\left\{\mathbf{G} \in X_{\ell} \backslash X_{\ell-1}: \operatorname{dim}\left(V \cdot W_{\mathbf{G}}\right) \leq r\right\}
$$

Theorem 2.1 shows that $Y_{r, \ell}$ is empty if $b / a \in \Psi_{N}$ and $r<b \ell / a$. More generally, we could ask for an upper bound on the dimension of $Y_{r, \ell}$ for all $r, \ell$, and if this estimate is strong enough the result for $k=1$ would follow.

Since this last question seems interesting in its own right, we phrase it in language that does not involve the notation from the proof of Theorem 2.5.

Problem 2.7. Let $V \subset S_{a}$ be a general linear series of dimension $N$. Estimate the dimension of

$$
\{W: \operatorname{dim}(V \cdot W) \leq r\} \subset \operatorname{Gr}\left(\ell, S_{b}\right)
$$

\subsection{Semistable pullbacks}

We are now ready to prove our result on the semistability of pullbacks of Steiner bundles to rational curves. The main observation is that the splitting type of a vector bundle on a rational curve is easy to detect cohomologically.

Theorem 2.8. Let $E$ be a general Steiner bundle on $\mathbb{P}^{N}$, given by a resolution

$$
0 \rightarrow \mathcal{O}_{\mathbb{P}^{N}}(-1)^{k s} \stackrel{M}{\rightarrow} \mathcal{O}_{\mathbb{P}^{N}}^{k(s+r)} \rightarrow E \rightarrow 0
$$


where $s / r \in \Phi_{N}$ and $M$ is given by a general matrix of linear forms. If $f: \mathbb{P}^{1} \rightarrow \mathbb{P}^{N}$ is a general degree $r$ map and $k$ is sufficiently large, then

$$
f^{*} E \cong \mathcal{O}_{\mathbb{P}^{1}}(s)^{k r}
$$

Proof. Suppose $f: \mathbb{P}^{1} \rightarrow \mathbb{P}^{N}$ is given by a general $(N+1)$-dimensional series $V \subset$ $H^{0}\left(\mathcal{O}_{\mathbb{P}^{1}}(r)\right)$. The bundle $f^{*} E$ fits into an exact sequence

$$
0 \rightarrow \mathcal{O}_{\mathbb{P}^{1}}(-r)^{k s} \stackrel{f^{*} M}{\rightarrow} \mathcal{O}_{\mathbb{P}^{1}}^{k(s+r)} \rightarrow f^{*} E \rightarrow 0
$$

and the map $f^{*} M$ is given by a general $k(s+r) \times k s$ matrix with entries in $V$. Observe that $c_{1}\left(f^{*} E\right)=k s r$, and thus

$$
f^{*} E \cong \bigoplus_{i=1}^{k r} \mathcal{O}_{\mathbb{P}^{1}}\left(a_{i}\right)
$$

for some numbers $a_{i}$ with $\sum a_{i}=k s r$. We will have $f^{*} E \cong \mathcal{O}_{\mathbb{P}^{1}}(s)^{k r}$ if and only if

$$
H^{0}\left(\left(f^{*} E\right)^{\vee}(s-1)\right)=0 .
$$

Dualizing the above exact sequence and twisting by $\mathcal{O}_{\mathbb{P}^{1}}(s-1)$, we get an exact sequence

$$
0 \rightarrow\left(f^{*} E\right)^{\vee}(s-1) \rightarrow \mathcal{O}_{\mathbb{P}^{1}}(s-1)^{k(s+r)} \rightarrow \mathcal{O}_{\mathbb{P}^{1}}(s+r-1)^{k s} \rightarrow 0,
$$

so $H^{0}\left(\left(f^{*} E\right)^{\vee}(s-1)\right)=0$ if and only if

$$
H^{0}\left(\mathcal{O}_{\mathbb{P}^{1}}(s-1)\right)^{k(s+r)} \rightarrow H^{0}\left(\mathcal{O}_{\mathbb{P}^{1}}(s+r-1)\right)^{k s}
$$

is injective. But $(r+s) / s \in \Psi_{N+1}$ since $s / r \in \Phi_{N}$, so Proposition 2.5 completes the proof.

As a consequence, we obtain the semistability of the above Steiner bundles.

Corollary 2.9. For sufficiently large $k$, the bundles of the previous theorem are semistable. Thus every slope $\mu \in \Phi_{N}$ is realized by a semistable Steiner bundle. 
Proof. In the notation of the theorem, if $F \subset E$ is a destabilizing subbundle, then $f^{*} F \subset$ $f^{*} E$ is also a destabilizing subbundle, so $E$ is semistable since $f^{*} E$ is.

This corollary is our contribution to the proof of Theorem 1.3 from the introduction; we will complete the proof in the next section.

\subsection{Slopes of semistable Steiner bundles}

To complete the proof of Theorem 1.3, we must show that if $\mu \notin \Phi_{N}$ then there is no semistable Steiner bundle of slope $\mu$. While it is not much of a stretch to derive this result from Brambilla [5], the result there is only stated for $\mathbb{P}^{2}$. Furthermore, the basic structure of the argument is interesting, and gives insight into Steiner bundles with slope $\mu<\phi_{N}$. We therefore sketch the argument, quoting results from Brambilla when necessary.

First of all, we fix $N$ and let $\left\{a_{n}\right\}$ be the sequence defined recursively by

$$
\begin{aligned}
a_{-1} & =0 \\
a_{0} & =1 \\
a_{n+1} & =(N+1) a_{n}-a_{n-1} .
\end{aligned}
$$

For $n \geq 0$, we define the Fibonacci bundle $F_{n}$ to be the general Steiner bundle with resolution

$$
0 \rightarrow \mathcal{O}_{\mathbb{P}^{N}}(-1)^{a_{n-1}} \stackrel{M}{\rightarrow} \mathcal{O}_{\mathbb{P}^{N}}^{a_{n}} \rightarrow F_{n} \rightarrow 0
$$

The bundles $F_{n}$ are exceptional (see [4]), so the isomorphism class of $F_{n}$ is constant as $M$ varies in an open set.

The following result follows from a trivial induction on $n$.

Lemma 2.10. We have $\mu\left(F_{n}\right)=\rho_{N}^{n}(0)$. 
It is worth recalling that $\rho_{N}^{n}(0)$ is an increasing sequence that converges to $\phi_{N}$. The main result we will need from Brambilla [5] is the following theorem concerning the structure of unstable general Steiner bundles.

Theorem 2.11 (Theorem $6.3[5]$ ). Let $E$ be a general Steiner bundle on $\mathbb{P}^{N}$, and suppose

$$
\mu\left(F_{n}\right) \leq \mu(E)<\mu\left(F_{n+1}\right) .
$$

There are uniquely determined integers $k_{1}$ and $k_{2}$ such that

$$
E \cong F_{n}^{k_{1}} \oplus F_{n+1}^{k_{2}}
$$

Proof of Theorem 1.3. If $E$ is a general Steiner bundle on $\mathbb{P}^{N}$ with slope $\mu \notin \Phi_{N}$, then $0<\mu<\phi_{N}$. Thus by the lemma and the theorem $E$ must be a direct sum of two bundles of different slopes, and $E$ is not semistable.

Notice that since any general Steiner bundle with exceptional slope is a direct sum of copies of a single Fibonacci bundle $F_{n}$, we can conclude from Theorem 2.11 that Theorem 2.8 holds for all $k \geq 1$ in case the slope is exceptional.

Corollary 2.12. Theorem 2.8 holds for all $k \geq 1$ in case $s / r$ is an exceptional slope.

Now that we have finished the classification of semistable slopes of Steiner bundles, it is possible to prove converses to Theorem 2.1 and Proposition 2.5.

Corollary 2.13. Let $V \subset S_{b-a}$ be a general $N$-dimensional series, and let $M$ be a general $a k \times b k$ matrix with entries in $V$. If the map

$$
S_{a-1}^{b k} \stackrel{M}{\rightarrow} S_{b-1}^{a k}
$$

is an isomorphism for some $k \geq 1$, then $b / a \in \Psi_{N}$. 
Proof. By the proofs of Theorem 2.8 and Corollary 2.9, the hypotheses imply there is a semistable Steiner bundle on $\mathbb{P}^{N-1}$ with slope $a /(b-a)$. By Theorem 1.3, $a /(b-a) \in \Phi_{N-1}$ and thus $b / a \in \Psi_{N}$.

Corollary 2.14. Suppose $V \subset S_{b-a}$ is an $N$-dimensional series such that $\mu_{V}(W) \geq b / a$ for every $W \subset S_{a-1}$, where $a$ and $b$ are coprime. Then $b / a \in \Psi_{N}$.

Proof. By the proof of Proposition 2.5, the general map

$$
S_{a-1}^{b k} \rightarrow S_{b-1}^{a k}
$$

given by a matrix with entries in $V$ is an isomorphism for $k$ sufficiently large. By the previous corollary, $b / a \in \Psi_{N}$. 


\section{Chapter 3}

\section{Basic geometry of the Hilbert scheme of points in the plane}

We begin our discussion of the Hilbert scheme of points in the plane by discussing some of its basic properties. We start with a discussion of the divisor and curve classes on $\mathscr{H}_{n}$ in Section 3.1. In the next section, we discuss some general results on the effective cone of $\mathscr{H}_{n}$. To make our intersection calculations totally rigorous, we check that several intersections are transverse in Section 3.3. While the required transversality results are easy to believe, we are unaware of a source where they are actually verified. This material will serve as a foundation for our construction of extremal effective divisors in the next chapter.

\subsection{Divisors and curves on $\mathscr{H}_{n}$}

The goal of this section is to understand the divisor and curve classes on $\mathscr{H}_{n}$, as well as their intersection properties. While the material in this section is likely well-known to experts, we include it for the sake of completeness. 
We begin by investigating the Picard group of $\mathscr{H}_{n}$. Recall that $\mathscr{H}_{n}$ carries two natural divisor classes $H$ and $\Delta$, where $H$ is the locus of subschemes meeting a fixed line and $\Delta$ is the locus of singular subschemes.

Lemma 3.1. The $\mathbb{Q}$-vector space Pic $\mathscr{H}_{n} \otimes \mathbb{Q}$ is spanned by $H$ and $\Delta$.

Proof. There is an exact sequence

$$
\begin{aligned}
\mathbb{Q} & \rightarrow \operatorname{Pic} \mathscr{H}_{n} \otimes \mathbb{Q} \rightarrow \operatorname{Pic}\left(\mathscr{H}_{n} \backslash \Delta\right) \otimes \mathbb{Q} \rightarrow 0 \\
1 & \mapsto \Delta,
\end{aligned}
$$

which shows that Pic $\mathscr{H}_{n} \otimes \mathbb{Q}$ is spanned by $\Delta$ and the preimage of generators of $\operatorname{Pic}\left(\mathscr{H}_{n} \backslash\right.$ $\Delta) \otimes \mathbb{Q}$, so it suffices to show that the restriction of $H$ to $\mathscr{H}_{n} \backslash \Delta \operatorname{spans} \operatorname{Pic}\left(\mathscr{H}_{n} \backslash \Delta\right) \otimes \mathbb{Q}$. We have $\mathscr{H}_{n} \backslash \Delta \cong\left(\operatorname{Sym}^{n} \mathbb{P}^{2}\right) \backslash \Delta^{\prime}$, where $\Delta^{\prime}$ is the locus of 0 -cycles supported at fewer than $n$ points. There is then an $n$ !-sheeted covering space

$$
p:\left(\mathbb{P}^{2}\right)^{n} \backslash \Delta^{\prime \prime} \rightarrow\left(\mathrm{Sym}^{n} \mathbb{P}^{2}\right) \backslash \Delta^{\prime}
$$

where $\Delta^{\prime \prime}$ is the evident locus. If $D$ is any divisor class on $\left(\operatorname{Sym}^{n} \mathbb{P}^{2}\right) \backslash \Delta^{\prime}$, then we see $p_{*} p^{*} D=n ! D$. It follows that the map

$$
p^{*}: \operatorname{Pic}\left(\left(\operatorname{Sym}^{n} \mathbb{P}^{2}\right) \backslash \Delta^{\prime}\right) \rightarrow \operatorname{Pic}\left(\left(\mathbb{P}^{2}\right)^{n} \backslash \Delta^{\prime \prime}\right)
$$

is injective mod torsion.

At this point, we need to understand the group $\operatorname{Pic}\left(\left(\mathbb{P}^{2}\right)^{n} \backslash \Delta^{\prime \prime}\right)$. Since $\Delta^{\prime \prime}$ has codimension 2 in $\left(\mathbb{P}^{2}\right)^{n}$ and $\left(\mathbb{P}^{2}\right)^{n}$ is smooth, it follows that $\operatorname{Pic}\left(\left(\mathbb{P}^{2}\right)^{n} \backslash \Delta^{\prime \prime}\right) \cong \operatorname{Pic}\left(\left(\mathbb{P}^{2}\right)^{n}\right)$. Recalling that $\operatorname{Pic}(X)=H^{1}\left(\mathcal{O}_{X}^{*}\right)$ for any smooth $X$, we consider the exponential sequence

$$
0 \rightarrow \mathbb{Z} \rightarrow \mathcal{O}_{\left(\mathbb{P}^{2}\right)^{n}} \rightarrow \mathcal{O}_{\left(\mathbb{P}^{2}\right)^{n}}^{*} \rightarrow 0
$$


on $\left(\mathbb{P}^{2}\right)^{n}$. The Künneth formula shows that $h^{1,0}\left(\left(\mathbb{P}^{2}\right)^{n}\right)=h^{2,0}\left(\left(\mathbb{P}^{2}\right)^{n}\right)=0$, so

$$
\operatorname{Pic}\left(\left(\mathbb{P}^{2}\right)^{n}\right) \cong H^{2}\left(\left(\mathbb{P}^{2}\right)^{n}, \mathbb{Z}\right) \cong \mathbb{Z}^{n}
$$

That is, the Picard group of $\left(\mathbb{P}^{2}\right)^{n}$ is generated by pullbacks of $\mathcal{O}_{\mathbb{P}^{2}}(1)$ along the $n$ possible projections.

The image of $p^{*}$ lies entirely in the $S_{n}$-equivariant portion of $\operatorname{Pic}\left(\left(\mathbb{P}^{2}\right)^{n} \backslash \Delta^{\prime \prime}\right)=\mathbb{Z}^{n}$. But $S_{n}$ acts on $\mathbb{Z}^{n}$ by permutation of the factors, so the equivariant divisors form a copy of $\mathbb{Z}$, generated by $p^{*} H$. It follows that $\operatorname{Pic}\left(\mathscr{H}_{n} \backslash \Delta\right) \otimes \mathbb{Q}$ is generated by $H$.

Dually, we would like to understand the curve classes on $\mathscr{H}_{n}$. Consider the following curves, each parameterized by a $\mathbb{P}^{1}$.

- $\alpha$ is the locus where $n-1$ points are fixed and the $n$th point moves on a fixed line disjoint from the $n-1$ other points.

- $\beta$ is the locus where $n-2$ points are fixed and a "spinning tangent vector" is supported at another fixed point.

- $\delta$ is the locus where $n-2$ points are fixed and a pair of points moves in a base point free linear pencil on a fixed line disjoint from the $n-2$ other points.

Since $\beta$ is contained in $\Delta$, it will be convenient to introduce one further divisor class for the purpose of computing the intersection pairing between curves and divisors:

- $D_{1}$ is the locus of schemes $\Gamma \in \mathscr{H}_{n}$ which meet some line in a subscheme of degree at least 3 .

The filled in entries of the following intersection table follow immediately from the definitions. 


\begin{tabular}{c|ccc} 
& $H$ & $\Delta$ & $D_{1}$ \\
\hline$\alpha$ & 1 & 0 & $\left(\begin{array}{c}n-1 \\
2\end{array}\right)$ \\
$\beta$ & 0 & & $n-2$ \\
$\delta$ & 1 & & $\left(\begin{array}{c}n-2 \\
2\end{array}\right)$
\end{tabular}

Notice that $\Delta$ is an extremal effective divisor since $\alpha$ is a moving curve dual to $\Delta$.

To compute $\delta \cdot \Delta$, let $L \subset \mathbb{P}^{2}$ be a line and let $\mathscr{D} \subset H^{0}\left(\mathcal{O}_{L}(2)\right)$ be a pencil. Singular members of this pencil correspond to branch points of the degree 2 map $L \rightarrow \mathbb{P}^{1}$ induced by $\mathscr{D}$. By Riemann-Hurwitz, there are exactly two such singular members, so $\delta$ meets $\Delta$ in two points. We will see in Section 3.3 that these intersections occur with multiplicity one, so in fact $\delta \cdot \Delta=2$. Since we know $H$ and $\Delta$ generate Pic $\mathscr{H}_{n} \otimes \mathbb{Q}$, this allows us to complete the intersection table.

\begin{tabular}{c|ccc} 
& $H$ & $\Delta$ & $D_{1}$ \\
\hline$\alpha$ & 1 & 0 & $\left(\begin{array}{c}n-1 \\
2\end{array}\right)$ \\
$\beta$ & 0 & -2 & $n-2$ \\
$\delta$ & 1 & 2 & $\left(\begin{array}{c}n-2 \\
2\end{array}\right)$
\end{tabular}

In particular, we conclude

$$
\begin{aligned}
D_{1} & =\left(\begin{array}{c}
n-1 \\
2
\end{array}\right) H-\frac{n-2}{2} \Delta \\
\delta & =\alpha-\beta .
\end{aligned}
$$

Modulo one relatively difficult fact, this discussion allows us to derive the full integral Picard group.

Theorem 3.2. The Picard group of $\mathscr{H}_{n}$ is

$\operatorname{Pic} \mathscr{H}_{n}=\mathbb{Z} H \oplus \mathbb{Z}(\Delta / 2)$ 
and the space of integral curve classes is

$$
A_{1}\left(\mathscr{H}_{n}\right)=\mathbb{Z} \alpha \oplus \mathbb{Z} \beta
$$

The intersection pairing Pic $\mathscr{H}_{n} \times A_{1}\left(\mathscr{H}_{n}\right) \rightarrow \mathbb{Z}$ is given by

\begin{tabular}{c|cc} 
& $H$ & $\Delta$ \\
\hline$\alpha$ & 1 & 0 \\
$\beta$ & 0 & -2
\end{tabular}

Proof. The difficult missing ingredient is the fact that $\operatorname{Pic}\left(\mathscr{H}_{n}\right)$ is torsion free, which is proved in Fogarty [11]. Granted that result, all we must do is determine which divisors $a H+b \Delta$ are in Pic $\mathscr{H}_{n}$, where $a, b \in \mathbb{Q}$. Intersecting with $\alpha$ and $\beta$ shows that if such a divisor lies in Pic $\mathscr{H}_{n}$ then $a \in \mathbb{Z}$ and $2 b \in \mathbb{Z}$. It remains to show that $\Delta / 2$ is in fact a divisor class. However, we shall see in Lemma 4.7 that if $\alpha: \Xi_{n} \rightarrow \mathscr{H}_{n}$ is the universal family, then $c_{1}\left(\alpha_{*} \mathcal{O}_{\Xi_{n}}\right)=-\Delta / 2$.

\subsection{General results on the effective cone}

In this section we collect two previously known results on the structure of the effective cone Eff $\mathscr{H}_{n}$. We thank Izzet Coskun for calling these results to our attention. The first part of the next result will also be useful in the study of the local geometry of the divisor $\Delta$ in the next section.

Proposition 3.3. Fix a point $q \in \mathbb{P}^{2}$, and define a rational map

$$
\begin{aligned}
i_{q}: \mathscr{H}_{n} & \rightarrow \mathscr{H}_{n+1} \\
\Gamma & \mapsto \Gamma \cup\{q\} .
\end{aligned}
$$


If $i_{q}^{*}:$ Pic $\mathscr{H}_{n+1} \rightarrow$ Pic $\mathscr{H}_{n}$ is the induced map on Picard groups, then $i_{q}^{*} H=H$ and $i_{q}^{*} \Delta=\Delta$, so Pic $\mathscr{H}_{n}$ and Pic $\mathscr{H}_{n+1}$ are naturally identified.

Furthermore, under this identification, Eff $\mathscr{H}_{n+1}$ is contained in Eff $\mathscr{H}_{n}$.

Proof. The claim that $i_{q}^{*} H=H$ is straightforward. To see $i_{q}^{*} \Delta=\Delta$, observe that $i_{q}^{-1}(\Delta)=$ $\Delta$ holds set-theoretically (as $i_{q}$ is defined everywhere except the codimension 2 locus of $\Gamma$ with $\{q\} \subset \Gamma)$. Thus we must show $i_{q}\left(\mathscr{H}_{n}\right)$ is generically transverse to $\Delta \subset \mathscr{H}_{n+1}$. Let $\Gamma \in \mathscr{H}_{n}$ be a point consisting of a degree 2 scheme and $n-2$ other points, and assume $\Gamma$ is disjoint from $q$. In terms of the identification

$$
T_{i_{q}(\Gamma)} \mathscr{H}_{n+1}=H^{0}\left(N_{\Gamma \cup\{q\} / \mathbb{P}^{2}}\right)=H^{0}\left(N_{\Gamma / \mathbb{P}^{2}}\right) \oplus H^{0}\left(N_{q / \mathbb{P}^{2}}\right)
$$

$i_{q *} T_{\Gamma} \mathscr{H}_{n}$ is just the subspace $H^{0}\left(N_{\Gamma / \mathbb{P}^{2}}\right)$. On the other hand, $T_{\Gamma \cup\{q\}} \Delta$ contains the subspace $H^{0}\left(N_{q / \mathbb{P}^{2}}\right)$. Since $\Gamma \cup\{q\}$ is a smooth point of $\Delta \subset \mathscr{H}_{n+1}$, it follows that $T_{\Gamma \cup\{q\}} \Delta$ cannot contain $H^{0}\left(N_{\Gamma / \mathbb{P}^{2}}\right)$, so $i_{q}\left(\mathscr{H}_{n}\right)$ is transverse to $\Delta \subset \mathscr{H}_{n+1}$ at $i_{q}(\Gamma)$.

For the statement on effective cones, let $D \subset \mathscr{H}_{n+1}$ be an effective divisor, and choose a reduced collection $p_{1}, \ldots, p_{n+1}$ of points such that the corresponding point in $\mathscr{H}_{n+1}$ does not lie in $D$. Then $D$ meets $i_{p_{1}}\left(\mathscr{H}_{n}\right)$ properly, so $i_{p_{1}}^{*}(D)$ is an effective divisor on $\mathscr{H}_{n}$ with the same class as $D$ under the identification of Picard groups.

The next proposition shows the effective cone is always closed, so it is reasonable to look for constructions of effective divisors spanning its edges.

Proposition 3.4. The Hilbert scheme $\mathscr{H}_{n}$ is a log Fano variety, hence a Mori dream space. In particular, the effective cone of $\mathscr{H}_{n}$ is closed.

Proof. By well-known results in birational geometry (see [14] for a survey with the relevant results and terminology), it is enough to show $\mathscr{H}_{n}$ is log Fano. The canonical class of $\mathscr{H}_{n}$ is 
$K_{\mathscr{H}_{n}}=-3 H$. Thus $-K_{\mathscr{H}_{n}}$ is nef, and since the nef cone is the closure of the ample cone we see $-\left(K_{\mathscr{H}_{n}}+\varepsilon \Delta\right)$ is ample for small $\varepsilon>0$. Furthermore, the pair $\left(\mathscr{H}_{n}, \varepsilon \Delta\right)$ is Kawamata $\log$ terminal for small $\varepsilon$ since $\mathscr{H}_{n}$ is smooth, so $\mathscr{H}_{n}$ is $\log$ Fano.

\subsection{The local geometry of $\Delta$}

In order to justify the calculation of intersection numbers of curves on $\mathscr{H}_{n}$ with the boundary divisor $\Delta$, it is necessary to better understand the local geometry of $\Delta$.

In this work, we will focus predominantly on a particular type of curve on $\mathscr{H}_{n}$. Let $C \subset \mathbb{P}^{2}$ be a smooth curve of degree $r$, and suppose we have a base-point free linear pencil $\mathscr{D}$ of degree $n$ on $C$. This pencil naturally induces a map

$$
\mathbb{P}^{1} \rightarrow C^{[n]} \subset \mathscr{H}_{n}
$$

so we get a curve $\gamma$ in $\mathscr{H}_{n}$. Evidently $\gamma \cdot H=r$. On the other hand, singular members of the pencil correspond to ramification points of the map $C \rightarrow \mathbb{P}^{1}$ induced by the pencil. In case this map is simply ramified and the corresponding intersections of $\gamma$ with $\Delta$ all have multiplicity one, Riemann-Hurwitz tells us that

$$
2 g(C)-2=n\left(2 g\left(\mathbb{P}^{1}\right)-2\right)+\gamma \cdot \Delta
$$

so

$$
\gamma \cdot \Delta=r(r-3)+2 n
$$

Our main goal for the section is to make this calculation rigorous, even in case the pencil $\mathscr{D}$ has base points, the ramification of the induced map $C \rightarrow \mathbb{P}^{1}$ is not simple, and/or the intersections of $\gamma$ with $\Delta$ are not transverse. By not having to verify these restrictive hypotheses, it becomes easier to construct curves of the desired class on $\mathscr{H}_{n}$. 
One approach to make this calculation rigorous is to give a detailed description of the tangent cone of the dual hypersurface of a curve embedded in projective space, and argue that the multiplicities of singularities in the dual hypersurface correspond to the ramification indices in the Riemann-Hurwitz formula; the framework for doing the calculation this way can be found in [17]. However, a somewhat less painful argument with fewer local computations is possible, which we present here.

Proposition 3.5. Let $\mathscr{D} \subset \mathbb{P} H^{0}(L)$ be an arbitrary linear pencil of degree $n$ on a smooth curve $C \subset \mathbb{P}^{2}$ of degree $r$. If $\gamma$ is the induced curve in $\mathscr{H}_{n}$, then

$$
\begin{aligned}
& \gamma \cdot H=r \\
& \gamma \cdot \Delta=r(r-3)+2 n .
\end{aligned}
$$

Proof. First suppose that $n \geq 2 g(C)+1$, so that $L$ automatically gives an embedding $C \subset \mathbb{P} H^{0}(L)^{*}$. In this case, we observe that the curve class $\gamma$ does not depend either on the choice of $L$ or the choice of pencil $\mathscr{D} \subset \mathbb{P} H^{0}(L)$. Indeed, the Abel-Jacobi map $C^{[n]} \rightarrow \operatorname{Pic}^{n} C$ realizes $C^{[n]}$ as a $\mathbb{P}^{N}$-bundle over $\operatorname{Pic}^{n} C$ for some $N$, where the fiber over $L$ is the complete series $\mathbb{P} H^{0}(L)$. We may then form the associated flag bundle over $\operatorname{Pic}^{n} C$ whose fiber over $L \in \operatorname{Pic}^{n} C$ consists of tuples $\sigma \in \mathscr{D} \subset \mathbb{P} H^{0}(L)$, where $\mathscr{D}$ is a pencil and $\sigma$ is a section. This flag bundle maps to $\mathscr{H}_{n}$, and letting $\sigma$ vary in a fixed pencil $\mathscr{D} \subset \mathbb{P} H^{0}(L)$ gives a curve of class $\gamma$; allowing the pencil to vary shows all these curves are equivalent. We may therefore assume that both $L$ and $\mathscr{D}$ are general.

Denote by $C^{*} \subset \mathbb{P} H^{0}(L)$ the dual variety of $C$, consisting of hyperplanes in $\mathbb{P} H^{0}(L)^{*}$ tangent to $C$. It is a reduced hypersurface. The pencil $\mathscr{D}$ is a line in $\mathbb{P} H^{0}(L)$, so $\mathscr{D} \cdot C^{*}$ equals the degree of $C^{*}$; since $\mathscr{D}$ is general, it meets $C^{*}$ transversely at $\operatorname{deg} C^{*}$ smooth points, all of which correspond to points in $C^{[n]}$ of the form $2 p_{1}+p_{2}+\cdots+p_{n-1}$. Then the 
map $C \rightarrow \mathbb{P}^{1}$ induced by $\mathscr{D}$ is simply ramified over $\operatorname{deg} C^{*}$ points, and thus

$$
2 g(C)-2=n\left(2 g\left(\mathbb{P}^{1}\right)-2\right)+\operatorname{deg} C^{*},
$$

from which we find $\operatorname{deg} C^{*}=r(r-3)+2 n$.

We now wish to conclude by push-pull that $\gamma \cdot \Delta=r(r-3)+2 n$. For this, we need to know that if $i: \mathbb{P} H^{0}(L) \rightarrow \mathscr{H}_{n}$ is the evident inclusion then $i^{*} \Delta=C^{*}$. We see this in two steps. We will show in Lemma 3.6 that if $j: C^{[n]} \rightarrow \mathscr{H}_{n}$ is the inclusion then $j^{*} \Delta=\Delta_{C[n]}$. Assuming this for now, observe that if $k: \mathbb{P} H^{0}(L) \rightarrow C^{[n]}$ is the inclusion then $k^{-1} \Delta_{C^{[n]}}=C^{*}$ holds set-theoretically. Furthermore, $k^{-1} \Delta_{C^{[n]}}$ is reduced. Let $U \subset \Delta_{C^{[n]}}$ be the smooth locus of the diagonal. Restricting the Abel-Jacobi map to $U$ gives a dominant map $U \rightarrow \operatorname{Pic}^{n} C$. By generic smoothness, the differential of this map is surjective at a general point of $U$. It follows that $k^{-1} \Delta_{C}[n]$ meets the generic fiber of the Abel-Jacobi map generically transversely, from which we conclude $k^{*} \Delta_{C[n]}=C^{*}$ and thus $i^{*} \Delta=C^{*}$. This completes the proof in case $\operatorname{deg} L \geq 2 g(C)+1$.

As a special case of what we have just shown, observe that if $\delta$ is the curve on $\mathscr{H}_{2}$ studied in the previous section, then $\delta \cdot \Delta=2$. It follows that $\beta \cdot \Delta=-2$. More generally, these intersection numbers hold on $\mathscr{H}_{n}$ by Proposition 3.3.

To finish the proof without the assumption that $n$ is large, we show that if $\mathscr{D}=\left\{D_{t}\right\}_{t \in \mathbb{P}^{1}}$ is a pencil and $p \in C$ is a general point, then the result holds for $\mathscr{D}$ if it holds for the pencil $\mathscr{D}+p$ that has a base-point. By adding many base-points, we reduce to the previous case.

Choose the point $p$ so that it is not a ramification point of the map $f: C \rightarrow \mathbb{P}^{1}$ induced by $\mathscr{D}$; without loss of generality assume that $f(p)=0$, so $p \in D_{0}$. Choose a general line 
$L \subset \mathbb{P}^{2}$ meeting $C$ transversely at $p$. We may then define a rational map

$$
\begin{aligned}
\mathbb{P}^{1} \times L & \rightarrow \mathscr{H}_{n+1} \\
(t, q) & \mapsto D_{t} \cup\{q\} .
\end{aligned}
$$

This map fails to be defined exactly at the point $(0, p)$ : as we approach $(0, p)$ from different directions in $\mathbb{P}^{1} \times L$, the limiting point in $\mathscr{H}_{n+1}$ is the $n-1$ points of $D_{0}$ other than $p$ unioned with any degree 2 subscheme of $\mathbb{P}^{2}$ supported at $p$. Thus blowing up $\mathbb{P}^{1} \times L$ at $(0, p)$ resolves the indeterminacy in this map, and the fiber over $q \in L$ is reducible, with one copy of $\mathbb{P}^{1}$ mapping to $\mathscr{H}_{n+1}$ according to $\mathscr{D}+p$ and another copy mapping to $\mathscr{H}_{n+1}$ as a curve of class $\beta$; the common intersection of these two curves maps to the unique scheme in the curve of class $\beta$ which lies in $C$.

The upshot is that the class $\gamma_{\mathscr{D}+p}$ is equivalent to $i_{q}\left(\gamma_{\mathscr{D}}\right)-\beta$, where $i_{q}: \mathscr{H}_{n} \rightarrow \mathscr{H}_{n+1}$ unions a scheme with a general fixed point $q$. By Proposition 3.3 and our calculation of $\Delta \cdot \beta$

$$
\gamma_{\mathscr{D}+p} \cdot \Delta=\gamma_{\mathscr{D}} \cdot \Delta+2
$$

which is the required equality.

We finish the section with the promised lemma.

Lemma 3.6. Let $C \subset \mathbb{P}^{2}$ be a smooth curve of degree $r$, and let $j: C^{[n]} \rightarrow \mathscr{H}_{n}$ be the inclusion. Then $j^{*} \Delta=\Delta_{C}[n]$.

Proof. To prove that $j^{*} \Delta=\Delta_{C}[n]$, it suffices to prove that $j_{*} C^{[n]}$ meets $\Delta$ generically transversely. To show this, we perform an explicit tangent space calculation. Suppose $C$ is defined by the equation $f(x, y)=0$ in affine local coordinates, and without loss of generality assume $C$ passes through the origin and is tangent to the $x$-axis there, so that $f$ lies in the 
ideal $\left(x^{2}, y\right) \subset \mathbb{C}[x, y]$. Let $\Gamma \in \mathscr{H}_{n}$ be the scheme corresponding to a union of $\Gamma^{\prime}=Z\left(x^{2}, y\right)$ and $n-2$ other points $p_{1}, \ldots, p_{n-2}$ lying on $C$. Recall that

$$
T_{\Gamma} \mathscr{H}_{n}=H^{0}\left(N_{\Gamma / \mathbb{P}^{2}}\right)=H^{0}\left(N_{\Gamma^{\prime} / \mathbb{P}^{2}}\right) \oplus H^{0}\left(N_{p_{1} / \mathbb{P}^{2}}\right) \oplus \cdots \oplus H^{0}\left(N_{p_{n-2} / \mathbb{P}^{2}}\right) .
$$

Clearly $T_{\Gamma} \Delta$ contains the subspace of $T_{\Gamma} \mathscr{H}_{n}$ spanned by all the $H^{0}\left(N_{p_{i} / \mathbb{P}^{2}}\right)$ summands. There is a similar decomposition for $T_{\Gamma} C^{[n]}$, so our computation amounts to showing that the image of $T_{\Gamma} \Delta$ in $H^{0}\left(N_{\Gamma^{\prime} / \mathbb{P}^{2}}\right)$ meets the subspace $H^{0}\left(N_{\Gamma^{\prime} / C}\right)$ transversely. As these subspaces are independent of $n$, it suffices to consider the case $n=2$, where $\Gamma=\Gamma^{\prime}$.

Now in case $n=2$, we identify $T_{\Gamma} \Delta$ and $H^{0}\left(N_{\Gamma / C}\right)$ as subspaces of $H^{0}\left(N_{\Gamma / \mathbb{P}^{2}}\right)$. We have

$$
H^{0}\left(N_{\Gamma / \mathbb{P}^{2}}\right)=\operatorname{Hom}_{\mathcal{O}_{\mathbb{P}^{2}}}\left(\mathscr{I}_{\Gamma}, \mathcal{O}_{\Gamma}\right)=\operatorname{Hom}_{\mathbb{C}[x, y]}\left(\left(x^{2}, y\right), \mathbb{C}[x] /\left(x^{2}\right)\right) .
$$

This last space is a 4 -dimensional $\mathbb{C}$-vector space, where $x^{2}$ and $y$ are independently able to map to any element of $\mathbb{C}[x] /\left(x^{2}\right)$. This last description is compatible with the description of $T_{\Gamma} \mathscr{H}_{n}$ as a subspace of the tangent space to the Grassmannian in which the Hilbert scheme embeds, so we can calculate tangent vectors by the standard approach for Grassmannians.

First let us identify $T_{\Gamma} \Delta$. As $\Delta$ is 3 -dimensional and smooth at $\Gamma$, we introduce three families of ideals corresponding to arcs in $\Delta$ and specializing to $I_{\Gamma}=\left(x^{2}, y\right)$ :

- $A_{t}=\left(x^{2}, y-t\right)$

- $B_{t}=\left((x-t)^{2}, y\right)$, and

- $C_{t}=\left(x^{2}, y-t x\right)$.

Each family of ideals induces an arc in $\Delta$, and we compute the homomorphisms $\varphi_{A}, \varphi_{B}, \varphi_{C}$ : $\left(x^{2}, y\right) \rightarrow \mathbb{C}[x] /\left(x^{2}\right)$ corresponding to their tangent vectors. 
- Put $v(t)=x^{2}$ and $w(t)=y-t$, so that $v$ and $w$ are extensions of $x^{2}$ and $y$ to nearby elements of $A_{t}$. Then

$$
\varphi_{A}\left(x^{2}\right)=v^{\prime}(0)=0 \quad \text { and } \quad \varphi_{A}(y)=w^{\prime}(0)=-1 .
$$

- Put $v(t)=(x-t)^{2}$ and $w(t)=y$, so that $v$ and $w$ are extensions of $x^{2}$ and $y$ to nearby elements of $B_{t}$. Then

$$
\varphi_{B}\left(x^{2}\right)=v^{\prime}(0)=-2 x \quad \text { and } \quad \varphi_{B}(y)=w^{\prime}(0)=0 .
$$

- Put $v(t)=x^{2}$ and $w(t)=y-t x$, so that $v$ and $w$ are extensions of $x^{2}$ and $y$ to nearby elements of $C_{t}$. Then

$$
\varphi_{C}\left(x^{2}\right)=v^{\prime}(0)=0 \quad \text { and } \quad \varphi_{C}(y)=w^{\prime}(0)=-x
$$

We observe that these three tangent vectors are independent, so they span $T_{\Gamma} \Delta$.

Our assumptions on $f$ (the local equation of $C$ ) imply that $(f, x)$ are analytic local coordinates for $\mathbb{C}^{2}$ near $(0,0)$. Also, we can find a holomorphic function $g(x)$ so that $f(x, g(x)) \equiv 0$ near $x=0$. We put $D_{t}=\left(x^{2}-t, y-g(x)\right)$, which specifies a holomorphic arc in $C^{[2]}$ limiting to $I_{\Gamma}$. Putting $v(t)=x^{2}-t$ shows

$$
\varphi_{D}\left(x^{2}\right)=v^{\prime}(0)=-1
$$

as $\varphi\left(x^{2}\right)$ never has a nonzero constant term for $\varphi \in T_{\Gamma} \Delta$, transversality follows. 


\section{Chapter 4}

\section{Effective divisors on the Hilbert scheme of points in the plane}

In this chapter we apply our results on Steiner bundles to construct effective divisors on $\mathscr{H}_{n}$. In the first section we show that certain vector bundles on $\mathbb{P}^{2}$ derived from Steiner bundles have interpolation for $n$ points, and we link the condition of interpolation to semistability. In the next section we derive a formula for the class of an effective divisor on $\mathscr{H}_{n}$ coming from a vector bundle that has interpolation for $n$ points, and we prove our main theorem on the effective cone.

For the remainder of the thesis, we write

$$
n=\frac{r(r+1)}{2}+s, \quad \text { with } 0 \leq s \leq r
$$

An overarching theme of our results is that the qualitative behavior of the effective cone of $\mathscr{H}_{n}$ depends primarily on the approximate value of the number $s / r \in[0,1]$, and not on either $s$ or $r$ itself. It is thus very useful to keep this decomposition of $n$ in mind. 


\subsection{Interpolation for bundles on $\mathbb{P}^{2}$}

In this section, we investigate when certain twists and/or duals of general Steiner bundles on $\mathbb{P}^{2}$ satisfy interpolation; in the next section, we will use this information to construct effective divisors on $\mathscr{H}_{n}$. We fix notation for this section in the following theorem.

Theorem 4.1. Write

$$
n=\frac{r(r+1)}{2}+s \quad(s \geq 0)
$$

and consider a general vector bundle E given by a resolution

$$
0 \rightarrow \mathcal{O}_{\mathbb{P}^{2}}(r-2)^{k s} \rightarrow \mathcal{O}_{\mathbb{P}^{2}}(r-1)^{k(s+r)} \rightarrow E \rightarrow 0 .
$$

For sufficiently large $k, E$ has interpolation for $n$ points if and only if $E$ is semistable, i.e. exactly when $s / r \in \Phi_{2}$.

Alternately, consider a general vector bundle $F$ given by a resolution

$$
0 \rightarrow F \rightarrow \mathcal{O}_{\mathbb{P}^{2}}(r)^{k(2 r-s+3)} \rightarrow \mathcal{O}_{\mathbb{P}^{2}}(r+1)^{k(r-s+1)} \rightarrow 0
$$

For sufficiently large $k, F$ has interpolation for $n$ points if and only if $F$ is semistable, i.e. exactly when

$$
1-\frac{s+1}{r+2} \in \Phi_{2}
$$

We will focus primarily on showing the result holds for bundles having the form of $E$ in the theorem, then indicate how analogous results for $F$ are proved. The following simple lemma plays a key role in showing semistable twisted Steiner bundles satisfy interpolation.

Lemma 4.2. With notation as in Theorem 4.1, if $C \subset \mathbb{P}^{2}$ is a curve of degree $r$, then the induced map

$$
H^{0}(E) \rightarrow H^{0}\left(\left.E\right|_{C}\right)
$$


is an isomorphism, and $H^{1}\left(\left.E\right|_{C}\right)=0$. Furthermore, $h^{0}(E)=k r n$.

Proof. Since $H^{1}(E)=0$ and there is an exact sequence

$$
\left.0 \rightarrow E(-r) \rightarrow E \rightarrow E\right|_{C} \rightarrow 0
$$

it suffices to show that $H^{0}(E(-r))=H^{1}(E(-r))=H^{2}(E(-r))=0$. This follows immediately from the sequence

$$
0 \rightarrow \mathcal{O}_{\mathbb{P}^{2}}(-2)^{k s} \rightarrow \mathcal{O}_{\mathbb{P}^{2}}(-1)^{k(s+r)} \rightarrow E(-r) \rightarrow 0
$$

The final statement is trivial.

Thus in order to show a bundle $E$ as above has interpolation, we may take the following approach. Choose some curve $C \subset \mathbb{P}^{2}$ of degree $r$, and show there are $n$ points $p_{1}, \ldots, p_{n} \in C$ such that $h^{0}\left(\left.E\right|_{C}\left(-p_{1}-\cdots-p_{n}\right)\right)=0$. It then follows that $E$ has no nonzero sections vanishing at $p_{1}, \ldots, p_{n}$. By choosing $C$ to be rational, we may apply our restriction results on Steiner bundles.

Proposition 4.3. With notation as in Theorem 4.1, if $k$ is sufficiently large and $E$ is semistable (i.e. if $s / r \in \Phi_{2}$ ), then $E$ has interpolation for $n$ points.

Proof. Since $h^{0}(E)=k r n$, we must only show that no nonzero sections of $E$ vanish at general points $p_{1}, \ldots, p_{n}$. Let $C \subset \mathbb{P}^{2}$ be a general rational curve of degree $r$. By the lemma, $H^{0}(E) \rightarrow H^{0}\left(\left.E\right|_{C}\right)$ is an isomorphism. Since $C$ is general, it has $(r-1)(r-2) / 2$ nodes. We specialize $(r-1)(r-2) / 2$ of our $n$ points onto the nodes of $C$, and specialize the remaining $2 r+s-1$ points onto smooth points of $C$. Denote by $D_{1}$ the divisor of the nodes of $C$ and by $D_{2}$ the divisor of the smooth points. 
Let $f: \mathbb{P}^{1} \rightarrow C$ be the normalization of $C$, given by a general net $V \subset H^{0}\left(\mathcal{O}_{\mathbb{P}^{1}}(r)\right)$, and let $\tilde{D}_{1}$ and $\tilde{D}_{2}$ be the divisors on $\mathbb{P}^{1}$ lying over $D_{1}$ and $D_{2}$, so that

$$
\operatorname{deg} \tilde{D}_{1}=2 \operatorname{deg} D_{1} \quad \text { and } \quad \operatorname{deg} \tilde{D}_{2}=\operatorname{deg} D_{2}
$$

Then

$$
H^{0}\left(f^{*}\left(\left.E\right|_{C}\right)\left(-\tilde{D}_{1}-\tilde{D}_{2}\right)\right) \cong H^{0}\left(\left.E\right|_{C}\left(-D_{1}-D_{2}\right)\right)
$$

By Theorem 2.8,

$$
f^{*}\left(\left.E(-(r-1))\right|_{C}\right) \cong \mathcal{O}_{\mathbb{P}^{1}}(s)^{k r}
$$

and therefore

$$
f^{*}\left(\left.E\right|_{C}\right) \cong \mathcal{O}_{\mathbb{P}^{1}}\left(r^{2}-r+s\right)^{k r}
$$

But

$$
\operatorname{deg}\left(\tilde{D}_{1}+\tilde{D}_{2}\right)=r^{2}-r+s+1
$$

so $H^{0}\left(f^{*}\left(\left.E\right|_{C}\right)\left(-\tilde{D}_{1}-\tilde{D}_{2}\right)\right)=H^{0}\left(\mathcal{O}_{\mathbb{P}^{1}}(-1)^{k r}\right)=0$.

On the other hand, we can show that if $E$ has interpolation then $E$ is semistable. The key tool is the following result for curves.

Lemma 4.4. Let $E$ be any vector bundle on a smooth curve $C$ with $h^{1}(E)=0$. If $E$ has interpolation for $n$ points, then $E$ is semistable.

Proof. If $E$ has rank $r$ and has interpolation, then $h^{0}(E)=r n$ and

$$
h^{0}(E \otimes L)=h^{1}(E \otimes L)=0
$$

for a general line bundle $L$ of degree $-r$. Thus $L$ is cohomologically orthogonal to $E$, and $E$ is semistable [9]. (An elementary argument using Riemann-Roch for vector bundles can also be given.) 
Proposition 4.5. With notation as in Theorem 4.1, if $E$ has interpolation, then $E$ is semistable.

Proof. If $s / r>1$ we have already seen that $E$ is semistable (regardless of whether it has interpolation), so we assume $s / r \leq 1$. Let $p_{1}, \ldots, p_{n} \in \mathbb{P}^{2}$ be general points such that $E$ has no nonzero sections vanishing at $p_{1}, \ldots, p_{n}$. Since $s \leq r$, there exists a smooth curve $C$ of degree $r$ that contains $p_{1}, \ldots, p_{n}$. By Lemma 4.2 , we have

$$
h^{0}\left(\left.E\right|_{C}\right)=k r n, \quad h^{0}\left(\left.E\right|_{C}\left(-p_{1}-\cdots-p_{n}\right)\right)=0, \quad \text { and } \quad h^{1}\left(\left.E\right|_{C}\right)=0,
$$

so $\left.E\right|_{C}$ has interpolation and Proposition 4.4 implies $\left.E\right|_{C}$ is semistable. But then $E$ must also be semistable.

Finally, we address what happens in the case of kernel bundles $F$.

Proposition 4.6. With notation as in Theorem 4.1, if $k$ is sufficiently large then $F$ has interpolation if and only if it is semistable.

Proof. The proof is almost identical to the one given for $E$ if one lets degree $r+2$ curves play the role of the degree $r$ curves in the proof for $E$. We also apply Theorem 2.8 to the dual of $F$ instead of to $F$. The only nontrivial point is that a priori we could have $h^{1}(F)>0$, and thus $h^{0}(F)>k(r+2) n$; however, assuming semistability holds the analogue of Proposition 4.3 shows that $h^{0}\left(F \otimes \mathscr{I}_{\Gamma}\right)=0$ for a general collection $\Gamma$ of $n$ points, which then forces $h^{0}(F)=k(r+2) n$.

\subsection{Effective divisors on $\mathscr{H}_{n}$}

In this section, we prove our main theorem on the effective cone of divisors on the Hilbert scheme of points in $\mathbb{P}^{2}$. We first show that a vector bundle $E$ that has interpolation gives 
rise to a divisor on the Hilbert scheme, given as the locus of subschemes where interpolation fails. We also compute the class of this divisor.

We begin by proving a simple result on the relation between vector bundles on $\mathbb{P}^{2}$ and bundles on $\mathscr{H}_{n}$.

Lemma 4.7. Let $E$ be a vector bundle of rank $r$ on $\mathbb{P}^{2}$, and let $\Xi_{n}$ be the universal family over $\mathscr{H}_{n}$, with maps as in the following diagram.

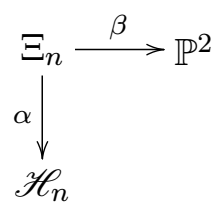

Then the bundle $E^{[n]}:=\alpha_{*} \beta^{*} E$ has rank rn and satisfies

$$
c_{1}\left(E^{[n]}\right)=c_{1}(E) H-\frac{\operatorname{rk} E}{2} \Delta .
$$

We remark that the transformation $E \mapsto E^{[n]}$ is known as a Fourier-Mukai transformation. The vector bundles on $\mathscr{H}_{n}$ of the form $E^{[n]}$ are known as the tautological bundles on $\mathscr{H}_{n}$.

Proof. We compute $\operatorname{ch}\left(E^{[n]}\right)$ using Grothendieck-Riemann-Roch. The map $\alpha$ is finite and generically simply ramified along the divisor

$$
\tilde{\Delta}=\left\{(\Gamma, p): p \in \Gamma_{\text {sing }}\right\} \subset \Xi_{n} \subset \mathscr{H}_{n} \times \mathbb{P}^{2},
$$

so $c_{1}\left(T_{\Xi_{n} / \mathscr{H}_{n}}\right)=-\tilde{\Delta}$, and we observe that $\alpha_{*}(\tilde{\Delta})=\Delta$. Also, if $\omega$ is a line class in $\mathbb{P}^{2}$, then $\alpha_{*} \beta^{*} \omega=H$, so $\alpha_{*} \beta^{*} c_{1}(E)=c_{1}(E) H$. But $R^{i} \alpha_{*} \beta^{*} E=0$ for $i>0$, so

$$
\begin{aligned}
\operatorname{ch}\left(E^{[n]}\right) & =\alpha_{*}\left(\operatorname{ch}\left(\beta^{*} E\right) \cdot \operatorname{Td}\left(T_{\mathscr{X} / \mathscr{H}_{n}}\right)\right) \\
& =\alpha_{*}\left(\left(r+\beta^{*} c_{1}(E)+\cdots\right)\left(1-\frac{1}{2} \tilde{\Delta}+\cdots\right)\right) \\
& =r n+c_{1}(E) H-\frac{r}{2} \Delta+\cdots,
\end{aligned}
$$


and we conclude $c_{1}\left(E^{[n]}\right)=c_{1}(E) H-\frac{r}{2} \Delta$.

We can use this lemma to give the class of an effective divisor corresponding to a bundle that has interpolation.

Proposition 4.8. Let $E$ be a vector bundle of rank $r$ on $\mathbb{P}^{2}$ that has interpolation for $n$ points. The locus of schemes $\Gamma \in \mathscr{H}_{n}$ such that $h^{0}\left(E \otimes \mathscr{I}_{\Gamma}\right) \neq 0$ forms an effective divisor $D_{E}$ in $\mathscr{H}_{n}$, and its class is

$$
\left[D_{E}\right]=c_{1}\left(E^{[n]}\right)=c_{1}(E) H-\frac{r}{2} \Delta
$$

Proof. Recall that the fiber of $E^{[n]}$ over a point $\Gamma \in \mathscr{H}_{n}$ is identified with the space $H^{0}\left(\left.E\right|_{\Gamma}\right)$. We have a natural map

$$
\psi: H^{0}(E) \otimes \mathcal{O}_{\mathscr{H}_{n}} \rightarrow E^{[n]}
$$

of vector bundles of rank $r n$ on $\mathscr{H}_{n}$, so the locus where $\psi$ fails to be an isomorphism has codimension at most 1 . Furthermore, $\psi$ fails to be an isomorphism exactly when $H^{0}(E) \rightarrow H^{0}\left(\left.E\right|_{\Gamma}\right)$ is not an isomorphism, and due to the sequence

$$
\left.0 \rightarrow E \otimes \mathscr{I}_{\Gamma} \rightarrow E \rightarrow E\right|_{\Gamma} \rightarrow 0
$$

this occurs exactly when $H^{0}\left(E \otimes \mathscr{I}_{\Gamma}\right) \neq 0$. Since $E$ has interpolation, the degeneracy locus of $\psi$ has codimension 1. Also, its class is given by $c_{1}\left(E^{[n]}\right)$.

We now fill in the last few steps in the proof of Theorem 1.8.

Proof of Theorem 1.8. From the resolutions

$$
\begin{aligned}
& 0 \rightarrow \mathcal{O}_{\mathbb{P}^{2}}(r-2)^{k s} \rightarrow \mathcal{O}_{\mathbb{P}^{2}}(r-1)^{k(s+r)} \rightarrow \quad \rightarrow \quad E \quad \rightarrow 0 \\
& 0 \rightarrow \quad F \quad \rightarrow \quad \mathcal{O}_{\mathbb{P}^{2}}(r)^{k(2 r-s+3)} \rightarrow \mathcal{O}_{\mathbb{P}^{2}}(r+1)^{k(r-s+1)} \rightarrow 0
\end{aligned}
$$


we compute

$$
\begin{aligned}
& c_{1}(E)=k\left(r^{2}-r+s\right), \\
& c_{1}(F)=k\left(r^{2}+r+s-1\right) .
\end{aligned}
$$

By Theorem 4.1 and Proposition 4.8, if $k$ is sufficiently large and $s$ and $r$ are such that $E$ (resp. $F$ ) is semistable, we get corresponding effective divisors $D_{E}\left(\right.$ resp. $D_{F}$ ) with classes

$$
\begin{aligned}
& {\left[D_{E}\right]=k\left(\left(r^{2}-r+s\right) H-\frac{r}{2} \Delta\right),} \\
& {\left[D_{F}\right]=k\left(\left(r^{2}+r+s-1\right) H-\frac{r+2}{2} \Delta\right) .}
\end{aligned}
$$

Let $\gamma \subset \mathscr{H}_{n}$ be the curve given by letting $n$ points move in a linear pencil on a smooth curve $C$ of degree $r$. Since $n<h^{0}\left(\mathcal{O}_{\mathbb{P}^{2}}(r)\right)$, a general collection of $n$ points lies on a smooth curve of degree $r$. Furthermore, Riemann-Roch asserts this collection moves in a linear pencil on $C$ since $n>g(C)$. Thus $\gamma$ is a moving curve on $\mathscr{H}_{n}$. Likewise, allowing $n$ points to move in a linear pencil on a smooth curve $C^{\prime}$ of degree $r+2$ also gives a moving curve $\gamma^{\prime}$ on $\mathscr{H}_{n}$ provided $s>1$ so that $n>g\left(C^{\prime}\right)=r(r+1) / 2$. By Proposition 3.5, we have

$$
\begin{aligned}
& \gamma \cdot H=r \quad \gamma \cdot \Delta=r(r-3)+2 n=2\left(r^{2}-r+s\right) \\
& \gamma^{\prime} \cdot H=r+2 \quad \gamma^{\prime} \cdot \Delta=(r+2)(r-1)+2 n=2\left(r^{2}+r+s-1\right) .
\end{aligned}
$$

We conclude that $\gamma \cdot D_{E}=\gamma^{\prime} \cdot D_{F}=0$, completing the proof. 


\section{Chapter 5}

\section{The remaining effective cones}

We conclude the thesis by providing partial results on the effective cone of $\mathscr{H}_{n}$ in cases not covered by Theorem 1.8. While $24 \%$ of all values of $n$ remain open, we can construct moving curves that conjecturally span the moving cone for about a third of the remaining cases. We also give conjectural constructions of effective divisors and moving curves for many open values of $n$. We then illustrate computer-aided proofs of these conjectures for small $n$, at least in a fixed positive characteristic.

\subsection{Summary of results and conjectures}

Let us briefly summarize an approximate picture of the current results and conjectures on the effective and moving cones. The actual results are slightly more messy, and are made precise in the indicated places. In particular, we neglect to mention the sporadic cases that can be handled via exceptional Steiner bundles and analogous concepts, and focus solely on cases corresponding to continuous behavior. 


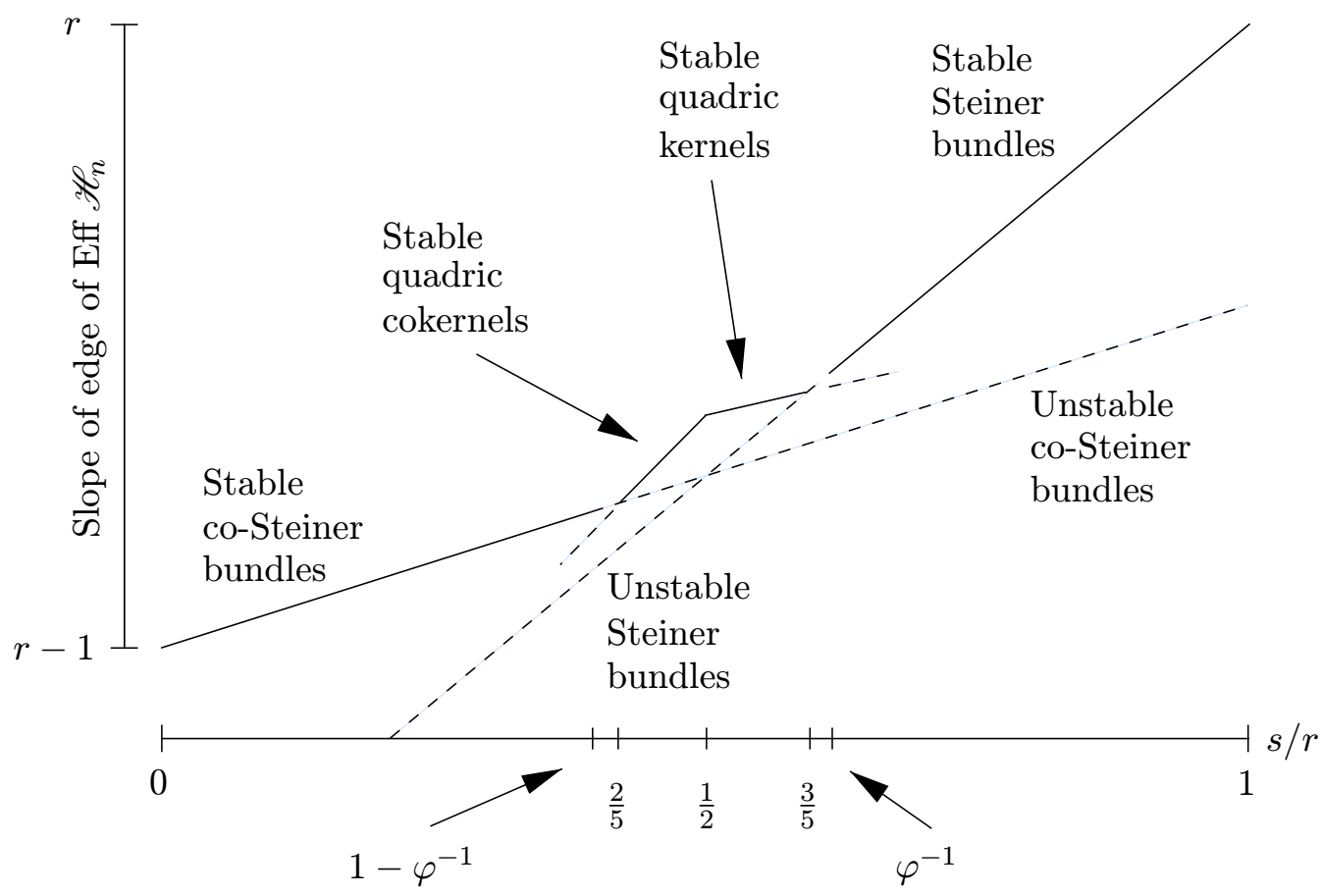

Figure 5.1: Conjectural schematic picture of the slope of the nontrivial edge of the effective cone of $\mathscr{H}_{n}$, where $n=r(r+1) / 2+s, r$ is fixed, and $s$ ranges from 0 to $r$. The image is distorted to emphasize the relative slopes between the lines; for large $r$ these slopes are all very similar.

Recall that

$$
n=\frac{r(r+1)}{2}+s \quad(0 \leq s \leq r)
$$

The structure of the effective cone of $\mathscr{H}_{n}$ predominately depends on the value of the ratio $s / r$, with only some slight inaccuracy; in the asymptotic picture as $r$ becomes large, this inaccuracy vanishes. Figure 5.1 summarizes the following discussion.

Case 1. $0<s / r<1-\varphi^{-1} \approx 0.382$. By Theorem 1.8 , the nontrivial extremal ray of 
the effective cone is spanned by

$$
\mu(E) H-\frac{1}{2} \Delta=\frac{r^{2}+r+s-1}{r+2} H-\frac{1}{2} \Delta,
$$

where $E$ is a vector bundle with resolution

$$
0 \rightarrow E \rightarrow \mathcal{O}_{\mathbb{P}^{2}}(r)^{2 r-s+3} \rightarrow \mathcal{O}_{\mathbb{P}^{2}}(r+1)^{r-s+1} \rightarrow 0
$$

so the extremal ray is given by Steiner kernel bundles. Dually, the moving curve is given by letting $n$ points move in a linear pencil on a smooth curve of degree $r+2$.

Case 2. $0.414 \approx \sqrt{2}-1<s / r<1 / 2$. There is a moving curve given by letting $n$ points move in a linear pencil on a curve of degree $2 r-1$ having

$$
m=r^{2}-(r-1)-n
$$

nodes and no further singularities - this construction works when $0<s / r<1 / 2$, but provides a moving curve of slope higher than the moving curve from Case 1 roughly when $s / r>0.4$ (Theorem 5.5). According to Conjecture 5.13, this moving curve is extremal, dual to the divisor given as the locus where interpolation fails for a general vector bundle with resolution

$$
0 \rightarrow \mathcal{O}_{\mathbb{P}^{2}}(r-3)^{s} \rightarrow \mathcal{O}_{\mathbb{P}^{2}}(r-1)^{2 r+s-1} \rightarrow E \rightarrow 0,
$$

a quadric cokernel bundle; interpolation is not known for these bundles. The extremal ray would be spanned by

$$
\frac{2 r^{2}-3 r+2 s+1}{2 r-1} H-\frac{1}{2} \Delta .
$$

Case 3. $1 / 2<s / r<2-\sqrt{2} \approx 0.586$. Dually to the previous case, we predict in Conjecture 5.14 that the edge of the effective cone corresponds to divisors coming from quadric kernel bundles, with resolution of the form

$$
0 \rightarrow E \rightarrow \mathcal{O}_{\mathbb{P}^{2}}(r)^{3 r-s+6} \rightarrow \mathcal{O}_{\mathbb{P}^{2}}(r+2)^{r-s+1} \rightarrow 0 .
$$


The moving curve should be given by allowing $n$ points to move in a linear pencil on a curve of degree $2 r+5$ having

$$
m=(r+3)^{2}-(r+2)-n
$$

nodes and no other singularities (the existence of this moving curve class is also not known). The extremal ray is spanned by

$$
\frac{2 r^{2}+3 r+2 s-2}{2 r+5} H-\frac{1}{2} \Delta .
$$

Case 4. $0.618 \approx \varphi^{-1}<s / r \leq 1$. Here Theorem 1.8 asserts the edge is spanned by the class

$$
\frac{r^{2}-r+s}{r} H-\frac{1}{2} \Delta
$$

corresponding to a Steiner cokernel bundle with resolution

$$
0 \rightarrow \mathcal{O}_{\mathbb{P}^{2}}(r-2)^{s} \rightarrow \mathcal{O}_{\mathbb{P}^{2}}(r-1)^{r+s} \rightarrow E \rightarrow 0
$$

Allowing $n$ points to move in a linear pencil on a smooth curve of degree $r$ yields the moving curve.

Remark 5.1. While the previous theorems and conjectures address the vast majority of all $n$, approximately $6.4 \%$ of all cases are completely open. There are a few natural guesses as to the slope of the effective cone for the remaining $n$, each generalizing the current data. For simplicity, let us focus on the case $1 / 2<s / r \leq 1$; the other cases have a dual picture.

Possibility 1. Given an $n$ with $1 / 2<s / r \leq 1$, we have constructed two different moving curve classes, as in Cases 3 and 4 above. Perhaps one of these two moving curve classes is always extremal.

If this is the case, then there exist $n$ such that the dual extremal effective divisors do not come from vector bundles satisfying interpolation, as we will discuss in Section 5.5. 
Possibility 2. We may need to allow more general vector bundles. Noting that in the solved and conjectured cases stable bundles have played an important role, we can choose a general stable vector bundle $E$ having minimal slope among vector bundles with $\chi(E)=n \cdot \mathrm{rk} E$. If such a bundle has interpolation, it may yield a divisor spanning the edge of the effective cone.

Drezet and Le Potier's classification in [6] of the possible numerical invariants of stable vector bundles allows one to determine the minimal slope in the preceding construction, and this slope agrees with the conjectured slope in the Steiner and quadric cases. A difficulty with this possibility is that the ranks of the minimal vector bundles become astronomical (in fact, are unbounded) when $s / r$ is around $3 / 5$, which means the dual moving curves would correspond to points moving on plane curves of very large degree.

Possibility 3. Finally, instead of allowing arbitrary stable vector bundles, perhaps we should only allow stable vector bundles where the minimal resolution of either the bundle or its dual is homogeneous, in the sense that the matrix consists entirely of forms of the same degree.

We feel that this option seems somewhat less natural, but it has the upside of allowing divisors to come from vector bundles while bounding the complexity of the situation around $s / r=3 / 5$.

Remark 5.2. The first case not handled by Steiner or quadric bundles is

$$
n=142=\frac{16 \cdot 17}{2}+6
$$

Already in this case, all three possibilities disagree. It is easy to check that Possibilities 1 , 
2 , and 3 predict edges spanned by

$$
\frac{277}{18} H-\frac{1}{2} \Delta, \quad \frac{1185}{77} H-\frac{1}{2} \Delta, \quad \text { and } \quad \frac{77}{5} H-\frac{1}{2} \Delta,
$$

respectively. Note in particular that if Possibility 2 is accurate, the dual moving curve involves 142 points moving on a curve of degree divisible by 77 .

\subsection{Existence of secant planes to curves}

The principal tool we will use to construct moving curves on $\mathscr{H}_{n}$ is the existence of higher secant planes to curves in projective space.

Theorem 5.3. Let $C \subset \mathbb{P}^{s}$ be a curve of degree $n$ and genus $g$. Then $C$ has d-secant $(d-r-1)$-planes if when we put

$$
\begin{aligned}
k & =s+1-d+r \\
\delta & =n-g-s
\end{aligned}
$$

we have

$$
\delta \geq 0, \quad r k \leq d, \quad \text { and } \quad(r-\delta) k \leq g .
$$

If we omit the hypothesis $(r-\delta) k \leq g$, this result appeared in [2, VIII.4, p. 355] as a consequence of the general secant plane formula; with this omission, however, the result is not true. For instance, without this hypothesis the theorem would imply a degree 4 elliptic curve in $\mathbb{P}^{3}$ possesses trisecant lines, which is false.

This result follows easily from the corresponding result for linear series on $C$, whose statement is actually a bit more applicable to our work. Let $\mathscr{D}=\mathbb{P} V \subset \mathbb{P} H^{0}(L)$ be a $g_{n}^{s}$ on $C$, and let $V_{d}^{r} \subset C^{[d]}$ be the locus of divisors of degree $d$ which impose at most $d-r$ conditions on $\mathscr{D}$. 
Theorem 5.4. Suppose

$$
\delta \geq 0, \quad r k \leq d, \quad \text { and } \quad(r-\delta) k \leq g,
$$

where $k$ and $\delta$ are as in Theorem 5.3. Then $V_{d}^{r}$ is nonempty. On the other hand, if

$$
\delta \geq 0, \quad r k \leq d, \quad \text { and } \quad(r-\delta) k>g,
$$

then either $V_{d}^{r}$ is empty or it does not have the expected dimension $d-r k$.

Proof. If $V_{d}^{r}$ is empty or has the expected dimension $d-r k \geq 0$, then the class $v_{d}^{r}$ of $V_{d}^{r}$ in rational cohomology is computed by the general secant plane formula

$$
v_{d}^{r}=\sum_{1 \leq \beta_{1}<\cdots<\beta_{k} \leq k+r} \Delta(\beta)^{2}\left(\prod_{i=1}^{k} \mu\left(r, k, \delta, i, \beta_{i}\right)\right) \theta^{\sum\left(\beta_{i}-i\right)} x^{r k-\sum\left(\beta_{i}-i\right)},
$$

where $\Delta(\beta)$ is the Vandermonde determinant corresponding to $\beta_{1}, \ldots, \beta_{k}$, the function $\mu$ is defined by

$$
\mu\left(r, k, \delta, i, \beta_{i}\right)=\left(\begin{array}{c}
\delta+i-1 \\
r+i-\beta_{i}
\end{array}\right) \frac{\left(r+i-\beta_{i}\right) !}{\left(r+k-\beta_{i}\right) !\left(\beta_{i}-1\right) !}
$$

$\theta$ is the pullback of the theta-divisor on $J(C)$ via the Abel-Jacobi map $C^{[d]} \rightarrow J(C)$, and $x$ is the class of the locus of $\Gamma \in C^{[d]}$ containing a fixed point of $C$ [2, VIII.4, p. 355]. The binomial coefficient in $\mu$ is defined for arbitrary integers $n$ and $i$ by the convention

$$
\left(\begin{array}{c}
n \\
i
\end{array}\right)= \begin{cases}\frac{n(n-1) \cdots(n-i+1)}{i !} & \text { if } i>0 \\
1 & \text { if } i=0 \\
0 & \text { if } i<0\end{cases}
$$

Fix a sequence $1 \leq \beta_{1}<\cdots<\beta_{k} \leq k+r$ corresponding to a single term

$$
\Delta(\beta)^{2}\left(\prod_{i=1}^{k} \mu\left(r, k, \delta, i, \beta_{i}\right)\right) \theta^{\sum\left(\beta_{i}-i\right)} x^{r k-\sum\left(\beta_{i}-i\right)}
$$


in the sum for $v_{d}^{r}$. Clearly $\Delta(\beta)^{2}$ is a nonzero positive number. For each $i$ with $1 \leq i \leq k$, we have $\delta+i-1 \geq 0$ since $\delta \geq 0$, so we have

$$
\left(\begin{array}{c}
\delta+i-1 \\
r+i-\beta_{i}
\end{array}\right) \geq 0
$$

and thus $\mu\left(r, k, \delta, i, \beta_{i}\right) \geq 0$ for all $i$. Notice that this binomial coefficient, and hence $\mu\left(r, k, \delta, i, \beta_{i}\right)$, vanishes precisely when

$$
\delta-1<r-\beta_{i}
$$

Thus the product $\prod_{i} \mu\left(r, k, \delta, i, \beta_{i}\right)$ is positive so long as $\delta-1 \geq r-\beta_{i}$ for all $i$, which occurs whenever $\delta-1 \geq r-\beta_{1}$. If in fact

$$
\beta_{1} \geq r-\delta+1
$$

then we must have

$$
\beta_{i} \geq r-\delta+i
$$

for all $i$.

When equality holds for all $i$, so $\beta_{i}=r-\delta+i$, the corresponding term reduces to a positive number times

$$
\theta^{k(r-\delta)} x^{k \delta}
$$

Since $x$ is ample on $C_{d}$ and $\theta$ is ample on $J(C)$, this cycle is nonempty and effective so long as $k(r-\delta) \leq g$, so that the power of $\theta$ does not exceed $g$. When this inequality holds, we conclude that the cycle $v_{d}^{r}$ is nontrivial, being a finite nonempty sum of positive effective terms, and thus $V_{d}^{r}$ is nonempty.

On the other hand, if $k(r-\delta)>g$, then we have

$$
\sum_{i=1}^{k}\left(\beta_{i}-i\right) \geq k(r-\delta)>g
$$


for any sequence $\left\{\beta_{i}\right\}$ such that $\prod_{i} \mu\left(r, k, \delta, i, \beta_{i}\right) \neq 0$. Since $\theta^{g+1}=0$, the corresponding term is zero, and thus $v_{d}^{r}=0$ when $V_{d}^{r}$ has the expected dimension.

\subsection{Some better moving curves on $\mathscr{H}_{n}$}

The goal of this section is to construct a family of highly-sloped moving curves on $\mathscr{H}_{n}$ for certain values of $n$ where we have not yet determined the edge of the effective cone.

Theorem 5.5. Write $n=r(r+1) / 2+s$, and suppose $0 \leq s<r / 2$. If $\Gamma \in \mathscr{H}_{n}$ is general, then there is a curve $C \subset \mathbb{P}^{2}$ of degree $2 r-1$ having

$$
m=r^{2}-(r-1)-n
$$

nodes and no other singularities, such that $\Gamma$ lies on smooth points of $C$ and $\Gamma$ moves in a linear pencil on the normalization $\tilde{C}$ of $C$. If $\gamma \subset \mathscr{H}_{n}$ is the corresponding moving curve class, then

$$
\begin{aligned}
& \gamma \cdot H=2 r-1 \\
& \gamma \cdot \Delta \geq 2\left(2 r^{2}-3 r+2 s+1\right),
\end{aligned}
$$

with equality whenever the pencil on $\tilde{C}$ has no member containing the full preimage of a node of $C$.

Note that

$$
m=\frac{1}{2}\left(r^{2}-3 r-2 s+2\right)
$$

so $0 \leq m<n$ since $s \geq 0$.

With $\gamma$ as in the theorem, if $E$ is a general vector bundle with resolution

$$
0 \rightarrow \mathcal{O}(r-3)^{s} \rightarrow \mathcal{O}(r-1)^{2 r+s-1} \rightarrow E \rightarrow 0
$$


and $E$ has interpolation for $n$ points, then $\gamma \cdot D_{E} \leq 0$, and equality is typically expected. We suspect such bundles $E$ satisfy interpolation so long as they are semistable, which occurs roughly when $\sqrt{2}-1<s / r<1 / 2$; we make this more precise in the next section. This moving curve has a bigger slope than the moving curve given by letting $n$ points move in a linear pencil on a smooth curve of degree $r+2$ so long as $s \geq \frac{1}{5}(2 r-1)$.

The key ingredient in the proof of the theorem is the study of a particular correspondence. Fix a line $L \subset \mathbb{P}^{2}$, and define

$$
\Sigma=\left\{\begin{array}{cc} 
& \Gamma \cup \Gamma^{\prime} \cup \Gamma^{\prime \prime} \text { is a reduced complete } \\
\left(\Gamma, \Gamma^{\prime}, \Gamma^{\prime \prime}\right): & \text { intersection of two } r \text {-ics, } \\
& \text { and }\left(\Gamma \cup \Gamma^{\prime}\right) \cap L=\emptyset
\end{array}\right\} \subset \mathscr{H}_{n} \times \mathscr{H}_{m} \times L^{[r-1]},
$$

noting that

$$
n+m+(r-1)=r^{2}
$$

The next proposition summarizes the relevant properties of $\Sigma$ for the proof of the theorem.

Proposition 5.6. If $n=r(r+1) / 2+s$ with $0 \leq s<r / 2$, then $\Sigma$

1. is irreducible,

2. dominates $\mathscr{H}_{n}$, and

3. dominates $\mathscr{H}_{m}$.

Let us first show that the proposition implies the theorem. We recall two facts for use in the proof.

Theorem 5.7 (Cayley-Bacharach [8]). Let $C_{1}, C_{2} \subset \mathbb{P}^{2}$ be plane curves of degrees d, e, and suppose that the intersection $\Gamma=C_{1} \cap C_{2}$ is zero-dimensional. Let $\Gamma^{\prime}$ and $\Gamma^{\prime \prime}$ be subschemes 
of $\Gamma$ residual to one another in $\Gamma$, and set $s=d+e-3$. If $k \leq s$ is a nonnegative integer, then the dimension of the family of curves of degree $k$ containing $\Gamma^{\prime}$ (modulo those containing all of $\Gamma$ ) is equal to the failure of $\Gamma^{\prime \prime}$ to impose independent conditions on curves of complementary degree $s-k$.

While we will only need the Cayley-Bacharach theorem in the classical case where $\Gamma$ is reduced, the full concept of residual schemes plays a role in the proof of Proposition 5.6. We recall that the subscheme $\Gamma^{\prime \prime}$ of $\Gamma$ residual to a subscheme $\Gamma^{\prime} \subset \Gamma$ is the scheme defined by the ideal sheaf

$$
\mathscr{I}_{\Gamma^{\prime \prime}}=\operatorname{Ann}\left(\mathscr{I}_{\Gamma^{\prime}} / \mathscr{I}_{\Gamma}\right)
$$

For arbitrary schemes this concept is not well-behaved; for instance if $\Gamma^{\prime} \subset \Gamma$ then the residual to the residual to $\Gamma^{\prime}$ in $\Gamma$ need not be $\Gamma^{\prime}$ again. However, when $\Gamma$ is Gorenstein (which in particular occurs whenever $\Gamma$ is a zero-dimensional complete intersection) everything works nicely.

The other result we will need describes the minimal resolution of the ideal sheaf of a general collection of $n$ points in $\mathbb{P}^{2}$.

Theorem 5.8 (Gaeta [7]). If $n=r(r+1) / 2+s$ with $0 \leq s \leq r$, then the ideal sheaf $\mathscr{I}_{\Gamma}$ of a general $\Gamma \in \mathscr{H}_{n}$ admits a resolution

$$
0 \rightarrow \mathcal{O}(-r-1)^{r-2 s} \oplus \mathcal{O}(-r-2)^{s} \rightarrow \mathcal{O}(-r)^{r-s+1} \rightarrow \mathscr{I}_{\Gamma} \rightarrow 0
$$

or

$$
0 \rightarrow \mathcal{O}(-r-2)^{s} \rightarrow \mathcal{O}(-r)^{r-s+1} \oplus \mathcal{O}(-r-1)^{2 s-r} \rightarrow \mathscr{I}_{\Gamma} \rightarrow 0
$$

depending on whether $s \leq r / 2$ or $s \geq r / 2$. In either case, the homogeneous ideal of $\Gamma$ is generated by $r$-ics and $(r+1)$-ics. 
Proof of Theorem 5.5. Since $\Sigma$ dominates $\mathscr{H}_{n}$, for a general $\Gamma \in \mathscr{H}_{n}$ we can find a triple $\left(\Gamma, \Gamma^{\prime}, \Gamma^{\prime \prime}\right) \in \Sigma$. Since $\Gamma \cup \Gamma^{\prime}$ is linked in a complete intersection of $r$-ics to the collinear collection $\Gamma^{\prime \prime}$, by Cayley-Bacharach the collection $\Gamma \cup \Gamma^{\prime}$ fails to impose independent conditions on curves of degree $2 r-4$. Then by Riemann-Roch, if there is a curve $C$ of degree $2 r-1$ passing through $\Gamma$, nodal at each point of $\Gamma^{\prime}$, and having no further singularities, then $\Gamma$ moves in a linear pencil on the normalization $\tilde{C}$ of $C$. Riemann-Hurwitz says that this linear pencil on $\tilde{C}$ has

$$
2 g(\tilde{C})-2-n\left(2 g\left(\mathbb{P}^{1}\right)-2\right)=2\left(2 r^{2}-3 r+2 s+1\right)
$$

singular members (with multiplicity). If $\gamma \subset \mathscr{H}_{n}$ is the induced curve in $\mathscr{H}_{n}$, it follows that

$$
\begin{aligned}
& \gamma \cdot H=2 r-1 \\
& \gamma \cdot \Delta \geq 2\left(2 r^{2}-3 r+2 s+1\right),
\end{aligned}
$$

with equality whenever no additional points of $\gamma \cap \Delta$ arise when the pencil descends from $\tilde{C}$ to $C$. We must therefore show that such a curve $C$ exists.

Consider the blowup $\mathrm{Bl}_{\Gamma \cup \Gamma^{\prime} \cup \Gamma^{\prime \prime}} \mathbb{P}^{2}$, and denote by $E, F, G$ the sums of exceptional divisors corresponding to $\Gamma, \Gamma^{\prime}$, and $\Gamma^{\prime \prime}$. By construction, the series $|r H-E-F-G|$ is nonempty and base point free. If we show that $|(r-1) H-F|$ is nonempty and base point free, then $|(2 r-1) H-E-2 F-G|$ is base-point free and its general member will be smooth by Bertini. Furthermore, the general curve of degree $r$ vanishing along $\Gamma \cup \Gamma^{\prime} \cup \Gamma^{\prime \prime}$ has general tangent directions at points of $\Gamma^{\prime}$ (since $\Gamma \cup \Gamma^{\prime} \cup \Gamma^{\prime \prime}$ is a transverse complete intersection of $r$-ics), so the general member of $|(2 r-1) H-E-2 F-G|$ meets each of the components of $F$ in a distinct pair of points. Thus the general member of this series corresponds to a plane curve of degree $2 r-1$ passing through $\Gamma$, nodal at each point of $\Gamma^{\prime}$, and having no further singularities. 
To finish the proof, we must therefore show $|(r-1) H-F|$ is nonempty and base-point free. Nonemptiness is obvious, as

$$
\operatorname{dim}|(r-1) H-F| \geq h^{0}\left(\mathcal{O}_{\mathbb{P}^{2}}(r-1)\right)-1-m=2 r+s-2,
$$

with equality whenever $\Gamma^{\prime}$ imposes independent conditions on curves of degree $r-1$. Thus we concentrate on base-point freeness.

We claim that if $X \subset \mathscr{H}_{m}$ is any proper subvariety, then for general $\Gamma \in \mathscr{H}_{n}$ we may find a triple $\left(\Gamma, \Gamma^{\prime}, \Gamma^{\prime \prime}\right) \in \Sigma$ such that $\Gamma^{\prime} \notin X$. Indeed, if $\beta: \Sigma \rightarrow \mathscr{H}_{m}$ is the projection, then since it is dominant we see that $U=\beta^{-1}\left(\mathscr{H}_{m} \backslash X\right) \subset \Sigma$ is a nonempty open subset. Since $\Sigma$ is irreducible, $U$ is a dense open subset. But since $\Sigma$ dominates $\mathscr{H}_{n}$, so does $U$, and the claim follows.

In particular, if we take $X \subset \mathscr{H}_{m}$ to be the locus of $\Gamma^{\prime}$ such that $|(r-1) H-F|$ is base-point free, it suffices to show that $X$ is a proper subvariety of the Hilbert scheme. For this it suffices to know that the general $\Gamma^{\prime} \in \mathscr{H}_{m}$ is cut out scheme-theoretically by $(r-1)$-ics, or that its ideal has a set of generators with degrees no more than $r-1$. But since $m<\left(\begin{array}{l}r \\ 2\end{array}\right)$, Gaeta's theorem implies the ideal of $\Gamma^{\prime}$ is generated by polynomials of degree at most $r-1$.

The most important aspect of $\Sigma$ for the previous proof is the fact that it dominates $\mathscr{H}_{n}$, as this is the condition that ensures we can find a potential location $\Gamma^{\prime}$ for the nodes of $C$. Unfortunately the full proof that $\Sigma$ dominates $\mathscr{H}_{n}$ is rather technical, even though the basic idea is simple. The next lemma contains the key insight of the proof, and also explains the occurrence of the condition $s<r / 2$.

Lemma 5.9. If $\Gamma \in \mathscr{H}_{n}$ is general and $0 \leq s<r / 2$, then there is some $\Gamma^{\prime \prime} \in L^{[r-1]}$ such that $\Gamma \cup \Gamma^{\prime \prime}$ lies on a pencil of $r$-ics. 
Proof. Let $V=\left.H^{0}\left(\mathscr{I}_{\Gamma}(r)\right)\right|_{L}$, and consider the linear series $\mathscr{E}=\mathbb{P} V$ on $L$. No curve in $\mathbb{P} H^{0}\left(\mathscr{I}_{\Gamma}(r)\right)$ contains $L$ since $\Gamma$ lies on no $(r-1)$-ic, so the restriction map $H^{0}\left(\mathscr{I}_{\Gamma}(r)\right) \rightarrow$ $V \subset H^{0}\left(\mathcal{O}_{L}(r)\right)$ is injective. Thus

$$
\operatorname{dim} \mathscr{E}=\left(\begin{array}{c}
r+2 \\
2
\end{array}\right)-1-n=r-s
$$

so $\mathscr{E}$ is a $g_{r}^{r-s}$ on the rational curve $L$. We must show that there is some divisor $\Gamma^{\prime \prime}$ on $L$ of degree $r-1$ such that $\mathscr{E}\left(-\Gamma^{\prime \prime}\right)$ has dimension at least 1 ; if we can do this then a lift of a pencil in $\mathscr{E}\left(-\Gamma^{\prime \prime}\right)$ to a pencil in $\mathbb{P} H^{0}\left(\mathscr{I}_{\Gamma}(r)\right)$ will be a pencil vanishing on $\Gamma \cup \Gamma^{\prime \prime}$.

Thus we wish to show that the locus $V_{r-1}^{s}$ of divisors $\Gamma^{\prime \prime} \subset L$ of degree $r-1$ which fail to impose at least $r-s$ conditions on $\mathscr{E}$ is nonempty. We denote by $\underline{s}, \underline{n}, \underline{r}, \underline{d}, \underline{g}, \underline{k}, \underline{\delta}$ the variables from Section 5.2, apologizing for the conflicts with our current notation. Then we have

$$
\begin{array}{llll}
\underline{s}=r-s & \underline{n}=r & \underline{r}=s \\
\underline{d}=r-1 & \underline{g}= & \\
\underline{k}=\underline{s}+1-\underline{d}+\underline{r}=2 & \underline{\delta}=\underline{n}-\underline{g}-\underline{s}=s . &
\end{array}
$$

The three inequalities

$$
\underline{\delta} \geq 0, \quad \underline{r} \underline{k} \leq \underline{d}, \quad \text { and } \quad(\underline{r}-\underline{\delta}) \underline{k} \leq \underline{g}
$$

are all satisfied since $0 \leq s<r / 2$, so in fact $V_{r-1}^{s}$ is nonempty by Theorem 5.4 .

Now that we have the lemma, assume $\Gamma \in \mathscr{H}_{n}$ is general, and find some $\Gamma^{\prime \prime} \in L^{[r-1]}$ such that $\Gamma \cup \Gamma^{\prime \prime}$ lies on a pencil of $r$-ics. Then we can let $\Gamma^{\prime}$ be the scheme residual to $\Gamma \cup \Gamma^{\prime \prime}$ in the base locus Bs $\mathscr{D}$. Assuming that Bs $\mathscr{D}$ is reduced and meets $L$ exactly in $\Gamma^{\prime \prime}$, the triple $\left(\Gamma, \Gamma^{\prime}, \Gamma^{\prime \prime}\right)$ lies in $\Sigma$, and we are done. Justifying this assumption requires substantial effort, however. 
Lemma 5.10. If $0 \leq s<r / 2$, then $\Sigma$ dominates $\mathscr{H}_{n}$.

Proof. We must introduce a couple auxiliary correspondences. First, let

$$
\mathscr{X}=\left\{\left(\Gamma^{\prime \prime}, \mathscr{D}\right): \mathscr{D} \in \operatorname{Gr}\left(2, H^{0}\left(\mathscr{I}_{\Gamma^{\prime \prime} \subset \mathbb{P}^{2}}(r)\right)\right)\right\} \subset L^{[r-1]} \times \operatorname{Gr}\left(2, H^{0}\left(\mathcal{O}_{\mathbb{P}^{2}}(r)\right)\right) .
$$

That is, $\mathscr{X}$ is the Grassmannian bundle over $L^{[r-1]}$ corresponding to 2-planes in the vector bundle on $L^{[r-1]}$ whose fiber over a point $\Gamma^{\prime \prime} \in L^{[r-1]}$ is $H^{0}\left(\mathscr{I}_{\Gamma^{\prime \prime} \subset \mathbb{P}^{2}}(r)\right)$. Since every $\Gamma^{\prime \prime} \in L^{[r-1]}$ imposes $r-1$ independent conditions on $r$-ics, Grauert's theorem [13, III.12.9] implies this vector bundle can be constructed as a pushforward. Clearly $\mathscr{X}$ is projective and irreducible. We must also consider the dense open subsets in $\mathscr{X}$ given by

$$
\begin{aligned}
& \mathscr{X}_{1}=\left\{\left(\Gamma^{\prime \prime}, \mathscr{D}\right): \text { Bs } \mathscr{D} \text { is zero-dimensional }\right\} \\
& \mathscr{X}_{2}=\left\{\left(\Gamma^{\prime \prime}, \mathscr{D}\right): \text { Bs } \mathscr{D} \text { is zero-dimensional, reduced, and Bs } \mathscr{D} \cap L=\Gamma^{\prime \prime}\right\} .
\end{aligned}
$$

It is easy to see that these are in fact dense open subsets of $\mathscr{X}$.

We now introduce the correspondence

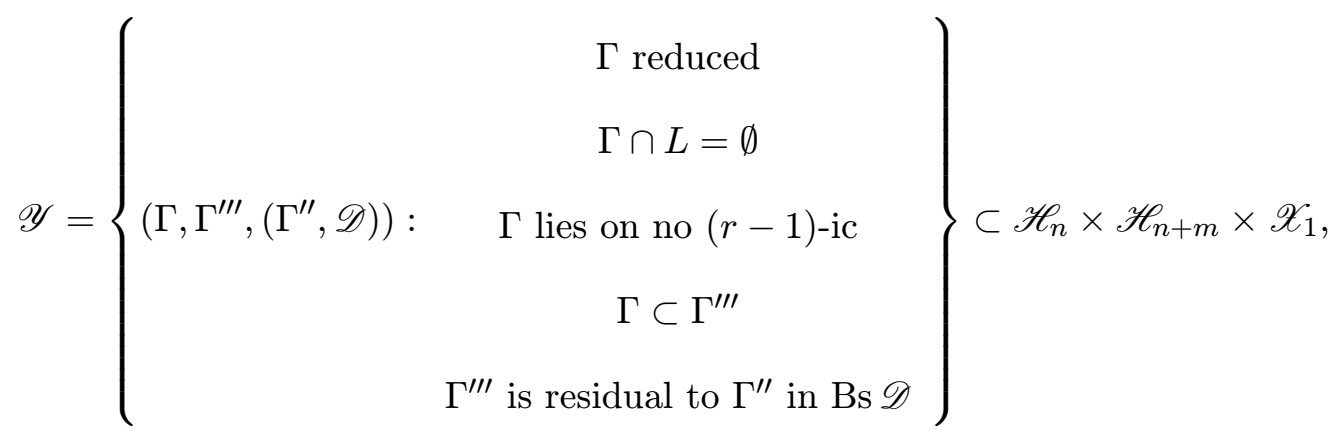

noting that $\mathscr{D}$ automatically has zero-dimensional base locus since $\Gamma \subset \operatorname{Bs} \mathscr{D}$ and $\Gamma$ lies on no $(r-1)$-ic, so that every member of $\mathscr{D}$ is irreducible.

Lemma 5.9 implies that $\mathscr{Y}$ dominates $\mathscr{H}_{n}$. We saw that for general $\Gamma \in \mathscr{H}_{n}$ there is some $\Gamma^{\prime \prime} \in L^{[r-1]}$ and a pencil $\mathscr{D}$ such that $\Gamma \cup \Gamma^{\prime \prime} \subset$ Bs $\mathscr{D}$. Now if we take $\Gamma^{\prime \prime \prime}$ to be residual to $\Gamma^{\prime \prime}$ in $\operatorname{Bs} \mathscr{D}$, the point $\left(\Gamma, \Gamma^{\prime \prime \prime},\left(\Gamma^{\prime \prime}, \mathscr{D}\right)\right)$ lies in $\mathscr{Y}$. 
Observe that we have a map

$$
\phi: \mathscr{X}_{1} \rightarrow \mathscr{H}_{n+m} \times \mathscr{X}_{1}
$$

given by sending a point $\left(\Gamma^{\prime \prime}, \mathscr{D}\right)$ to $\left(\Gamma^{\prime \prime \prime},\left(\Gamma^{\prime \prime}, \mathscr{D}\right)\right)$, where $\Gamma^{\prime \prime \prime}$ is the scheme residual to $\Gamma^{\prime \prime}$ in Bs $\mathscr{D}$. If

$$
\alpha: \mathscr{Y} \rightarrow \mathscr{H}_{n+m} \times \mathscr{X}_{1}
$$

is the projection, then we see that

$$
\alpha^{-1}\left(\phi\left(\mathscr{X}_{1}\right)\right)=\mathscr{Y}
$$

We claim that $\alpha^{-1}\left(\phi\left(\mathscr{X}_{2}\right)\right)$ contains a dense open subset of $\mathscr{Y}$; if this is true then $\alpha^{-1}\left(\phi\left(\mathscr{X}_{2}\right)\right)$ will dominate $\mathscr{H}_{n}$, which implies $\Sigma$ dominates $\mathscr{H}_{n}$.

Let $\left(\Gamma, \Gamma^{\prime \prime \prime},\left(\Gamma^{\prime \prime}, \mathscr{D}\right)\right)$ be any point in $\mathscr{Y}$, and choose a general curve $\gamma(t)=\left(\Gamma_{t}^{\prime \prime}, \mathscr{D}_{t}\right)$ in $\mathscr{X}$ with $\gamma(0)=\left(\Gamma^{\prime \prime}, \mathscr{D}\right)$, parameterized by a disc $\Delta$. For small nonzero $t$, we have $\gamma(t) \in \mathscr{X}_{2}$, so that the scheme $\Gamma_{t}^{\prime \prime \prime}$ residual to $\Gamma_{t}^{\prime \prime}$ in $\mathrm{Bs} \mathscr{D}_{t}$ is a reduced collection of points disjoint from $\Gamma_{t}^{\prime \prime}$. After a base change if necessary, we may assume that there are $n+m \operatorname{arcs} p_{i}(t)$ in $\mathbb{P}^{2}$ parameterized by $\Delta$ such that $\Gamma_{t}^{\prime \prime \prime}=\left\{p_{1}(t), \ldots, p_{n+m}(t)\right\}$ for nonzero $t$. Without loss of generality we may assume $\Gamma=\left\{p_{1}(0), \ldots, p_{n}(0)\right\}$; the assumption that $\Gamma$ is reduced is crucial here. Then if we write $\Gamma_{t}=\left\{p_{1}(t), \ldots, p_{n}(t)\right\}$, we obtain an arc

$$
\begin{aligned}
\tilde{\gamma}: \Delta & \rightarrow \mathscr{Y} \\
t & \mapsto\left(\Gamma_{t}, \Gamma_{t}^{\prime \prime \prime},\left(\Gamma_{t}^{\prime \prime}, \mathscr{D}_{t}\right)\right)
\end{aligned}
$$

such that $\tilde{\gamma}(0)=\left(\Gamma, \Gamma^{\prime \prime \prime},\left(\Gamma^{\prime \prime}, \mathscr{D}\right)\right)$ and $\tilde{\gamma}(t)$ lies in $\alpha^{-1}\left(\phi\left(\mathscr{X}_{2}\right)\right)$ for small nonzero $t$. Thus $\alpha^{-1}\left(\phi\left(\mathscr{X}_{2}\right)\right)$ is dense in $\mathscr{Y}$, completing the proof.

The proof that $\Sigma$ dominates $\mathscr{H}_{m}$ is much easier. 
Lemma 5.11. If $0 \leq s<r / 2$, then $\Sigma$ dominates $\mathscr{H}_{m}$.

Proof. Note that $m<n$. If $\Gamma^{\prime} \in \mathscr{H}_{m}$ is general, choose a general $\Gamma_{0} \in \mathscr{H}_{n-m}$. Then $\Gamma^{\prime} \cup \Gamma_{0}$ is general in $\mathscr{H}_{n}$, so since $\Sigma$ dominates $\mathscr{H}_{n}$ we can find some triple $\left(\Gamma^{\prime} \cup \Gamma_{0}, \Gamma_{1}, \Gamma^{\prime \prime}\right) \in \Sigma$. Clearly then $\left(\Gamma_{0} \cup \Gamma_{1}, \Gamma^{\prime}, \Gamma^{\prime \prime}\right) \in \Sigma$, so $\Sigma$ dominates $\mathscr{H}_{m}$.

We conclude the proof of the proposition by showing $\Sigma$ is irreducible.

Lemma 5.12. If $0 \leq s<r / 2$, then $\Sigma$ is irreducible. In fact, if we have

$$
n=r(r+1) / 2+s
$$

and only assume $s \geq 0$, then $\Sigma$ is irreducible so long as $m \geq 0$, so that the definition of $\Sigma$ makes sense.

Proof. Let us put

$$
\Xi=\left\{\left(p,\left(\Gamma^{\prime \prime}, \mathscr{D}\right)\right): p \in \operatorname{Bs} \mathscr{D}\right\} \subset\left(\mathbb{P}^{2} \backslash L\right) \times \mathscr{X}_{2},
$$

and observe that $\Xi$ is an $(n+m)$-sheeted covering space of $\mathscr{X}_{2}$ (see the proof of Lemma 5.10 for the definition of $\left.\mathscr{X}_{2}\right)$. Denote by

$$
\Xi(k)=\left\{\left(p_{1}, \ldots, p_{k},\left(\Gamma^{\prime \prime}, \mathscr{D}\right)\right): p_{i} \in \mathrm{Bs} \mathscr{D} \text { distinct }\right\} \subset\left(\left(\mathbb{P}^{2} \backslash L\right)^{k} \backslash \Delta\right) \times \mathscr{X}_{2} .
$$

If we show $\Xi(n+m)$ is irreducible, then $\Sigma$ is irreducible since it is the image of $\Xi(n+m)$ under the map

$$
\left(p_{1}, \ldots, p_{m+n},\left(\Gamma^{\prime \prime}, \mathscr{D}\right)\right) \mapsto\left(\left\{p_{1}, \ldots, p_{n}\right\},\left\{p_{n+1}, \ldots, p_{n+m}\right\}, \Gamma^{\prime \prime}\right)
$$

As $\Xi(n+m)$ is an étale $(m+n)$ !-sheeted cover of the irreducible variety $\mathscr{X}_{2}$, it suffices to show that $\Xi(n+m)$ is connected. Equivalently, we can show that the monodromy group 
of $\Xi \rightarrow \mathscr{X}_{2}$ is the full symmetric group. To do this, we show the monodromy acts doubly transitively on a fiber and that it contains a simple transposition.

To see the monodromy acts doubly transitively, it suffices to show that $\Xi(2)$ is connected. Consider the slightly enlarged correspondence

$$
\tilde{\Xi}(2)=\left\{\left(p_{1}, p_{2},\left(\Gamma^{\prime \prime}, \mathscr{D}\right)\right): p_{i} \in \operatorname{Bs} \mathscr{D} \text { distinct }\right\} \subset\left(\left(\mathbb{P}^{2} \backslash L\right)^{2} \backslash \Delta\right) \times \mathscr{X},
$$

observing in particular that we have allowed $\mathscr{D}$ to be an arbitrary pencil containing $\Gamma^{\prime \prime}$. Then the fiber of $\tilde{\Xi}(2)$ over the triple $\left(p_{1}, p_{2}, \Gamma^{\prime \prime}\right)$ consists of the Grassmannian

$$
\operatorname{Gr}\left(2, H^{0}\left(\mathscr{I}_{\left\{p_{1}, p_{2}\right\} \cup \Gamma^{\prime \prime}}(r)\right)\right) .
$$

Observing that the dimension $h^{0}\left(\mathscr{I}_{\left\{p_{1}, p_{2}\right\} \cup \Gamma^{\prime \prime}}(r)\right)$ is independent of $p_{1}, p_{2}$, and $\Gamma^{\prime \prime}$, we conclude by Grauert's theorem that $\tilde{\Xi}(2)$ is actually the Grassmannian bundle associated to a vector bundle on the irreducible variety $\left(\mathbb{P}^{2} \backslash L\right)^{2} \backslash \Delta$. Thus $\tilde{\Xi}(2)$ is irreducible, and the dense open subset $\Xi(2)$ is connected.

To find a simple transposition, consider the following family of pencils of $r$-ics, corresponding to a real loop in $\mathscr{X}_{2}$. Fix $r-1$ points $\Gamma^{\prime \prime}$ on $L$, let $C_{1}$ be a general curve of degree $r$ containing $\Gamma^{\prime \prime}$, and let $C_{2}$ be a general curve of degree $r-1$ containing $\Gamma^{\prime \prime}$. Pick a general tangent line to $C_{1}$, and choose affine coordinates on $\mathbb{P}^{2}$ so that this line is given by $y=0$ and tangent to $C_{1}$ at the origin. Fix a small $\varepsilon$, and denote by $L_{t}$ the line given by $y=\varepsilon e^{2 \pi i t}$. Put $\mathscr{D}_{t}=\left\langle C_{1}, C_{2} \cup L_{t}\right\rangle$. As $t$ goes from 0 to 1 , this gives a loop in $\mathscr{X}_{2}$ based at $\left\langle C_{1}, C_{2} \cup L_{0}\right\rangle$. The local equation of $C_{1}$ near the origin is $y=x^{2}$, so we see that the induced element of the monodromy group exchanges the pair of points of $L_{0} \cap C_{1}$ near the origin with one another while leaving all other base points of $\mathscr{D}_{0}$ fixed. Thus the monodromy group contains a simple transposition, and is the full symmetric group. 


\subsection{The quadric resolution conjectures}

In case

$$
\frac{s}{r-\frac{1}{2}}>\sqrt{2}-1 \approx 0.414
$$

the vector bundle $E$ with general resolution

$$
0 \rightarrow \mathcal{O}(r-3)^{s} \rightarrow \mathcal{O}(r-1)^{2 r+s-1} \rightarrow E \rightarrow 0
$$

will be stable by [5], and we have $h^{0}(E)=n \mathrm{rk} E$. These bundles are also stable in case $s /\left(r-\frac{1}{2}\right)$ is a convergent in the continued fraction expansion of $\sqrt{2}-1$; these bundles are analogs of the Steiner bundles with exceptional slopes. We saw in the last section that if $E$ has interpolation for $n$ points and $s / r<1 / 2$ then there is a moving curve on $\mathscr{H}_{n}$ dual to the divisor $D_{E}$.

Conjecture 5.13. Suppose

$$
\sqrt{2}-1<\frac{s}{r-\frac{1}{2}} \leq \frac{1}{2}
$$

or $s /\left(r-\frac{1}{2}\right)$ is a convergent in the continued fraction expansion of $\sqrt{2}-1$. Then the bundle $E$ above satisfies interpolation for $n$ points, so the class $\left[D_{E}\right]$ spans an edge of Eff $\mathscr{H}_{n}$.

Note that, at least for small $n$, computers are relatively good at checking whether bundles satisfy interpolation or not, at least over a finite field (coefficient explosion makes the calculation infeasible over $\mathbb{Q}$ ). For instance, the Macaulay2 script in Figure 5.2 verifies that the vector bundle on $\mathbb{P}_{\mathbb{F}_{7919}}^{2}$ with resolution

$$
0 \rightarrow \mathcal{O}_{\mathbb{P}^{2}}(4)^{3} \stackrel{M}{\rightarrow} \mathcal{O}_{\mathbb{P}^{2}}(6)^{16} \rightarrow E \rightarrow 0
$$

satisfies interpolation for 31 points in about 10 seconds. The program is successful in showing $E$ satisfies interpolation if it outputs "True." 




Figure 5.2: Macaulay2 script for checking interpolation for a quadric resolution cokernel bundle. 
An affirmative answer to this conjecture would compute Eff $\mathscr{H}_{n}$ in another $8.6 \%$ of all cases. In the cases where $s / r$ is just larger than $1 / 2$, there is a similar conjecture, which we now explain. Suppose that $s / r>1 / 2$ and

$$
\frac{r-s+1}{r+\frac{5}{2}}>\sqrt{2}-1
$$

which approximately says that $s / r<2-\sqrt{2} \approx 0.586$. In this case the vector bundle $F$ with resolution

$$
0 \rightarrow F \rightarrow \mathcal{O}_{\mathbb{P}^{2}}(r)^{3 r-s+6} \rightarrow \mathcal{O}_{\mathbb{P}^{2}}(r+2)^{r-s+1} \rightarrow 0
$$

is stable.

Conjecture 5.14. Suppose $s>r / 2$ and either

$$
\frac{r-s+1}{r+\frac{5}{2}}>\sqrt{2}-1
$$

or $(r-s+1) /\left(r+\frac{5}{2}\right)$ is a convergent in the continued fraction expansion of $\sqrt{2}-1$. Then the vector bundle $F$ above has interpolation for $n$ points. Furthermore, there is a moving curve on $\mathscr{H}_{n}$ given by allowing $n$ points to move in a linear pencil on a curve of degree $2 r+5$ having

$$
m=\frac{1}{2}\left(r^{2}+9 r-2 s+14\right)
$$

nodes, and its class is dual to $D_{F}$.

A trivial modification of the previous script can be used to test when $F$ has interpolation. A potential construction of the moving curves in this conjecture works very similarly to the construction in the previous section. Specifically, consider the correspondence

$$
\Sigma=\left\{\begin{array}{cc} 
& \Gamma^{\prime} \cup \Gamma \cup \Gamma^{\prime \prime} \text { is a reduced complete } \\
\left(\Gamma^{\prime}, \Gamma, \Gamma^{\prime \prime}\right): & \text { intersection of two }(r+3) \text {-ics, } \\
\text { and }\left(\Gamma^{\prime} \cup \Gamma\right) \cap L=\emptyset
\end{array}\right\} \subset \mathscr{H}_{m} \times \mathscr{H}_{n} \times L^{[r+2]},
$$


noting that

$$
n+m+(r+2)=(r+3)^{2}
$$

We have reversed the order of the $\mathscr{H}_{m}$ and $\mathscr{H}_{n}$ factors from the last section, for the reason that $m$ lies in the lower half of the range of numbers between two triangular numbers. Explicitly, we have

$$
m=\frac{(r+3)(r+4)}{2}+(r-s+1)=\frac{r^{\prime}\left(r^{\prime}+1\right)}{2}+s^{\prime}
$$

where $r^{\prime}=r+3$ and $s^{\prime}=r-s+1$, and it follows from $s>r / 2$ that $s^{\prime} / r^{\prime}<r^{\prime} / 2$. Thus $\Sigma$ is precisely the correspondence we constructed to study moving curves on $\mathscr{H}_{m}$ in the previous section. It therefore dominates both $\mathscr{H}_{m}$ and $\mathscr{H}_{n}$ by Proposition 5.6, and is irreducible as well.

The only sticking point when trying to mimic the proof from the last section to produce a moving curve on $\mathscr{H}_{n}$ is that the proof that there exists a curve nodal at $\Gamma^{\prime}$ and passing through $\Gamma$ does not work, as the series of curves of degree $r+2$ passing through $\Gamma^{\prime}$ is typically empty, and thus Bertini's theorem cannot be used to prevent additional singularities. Observe that the expected dimension of the series of curves of degree $2 r+5$ passing through $\Gamma$ and nodal at $\Gamma^{\prime}$ is

$$
\left(\begin{array}{c}
2 r+7 \\
2
\end{array}\right)-1-n-3 m=2 s-r-1
$$

which is always (at least just barely) nonnegative under our assumption $s>r / 2$. For instance, in the case $n=32$, we have $m=59$ and the expected dimension of curves passing through $n$ points and nodal at $m$ points is zero - we expect there to be a unique such curve. No Bertini-type argument which argues curves are smooth by producing reducible curves can possibly work, as this unique curve is typically irreducible. 
Resolving this issue seems to be a difficult interpolation-type problem. Even asserting that for a general $\left(\Gamma^{\prime}, \Gamma, \Gamma^{\prime \prime}\right) \in \Sigma$ the series of curves double at $\Gamma^{\prime}$ and containing $\Gamma$ has the expected dimension is not at all obvious, as $\Gamma$ and $\Gamma^{\prime}$, while general separately, depend closely on one another.

However, our knowledge of $\Sigma$ allows us to at least attack specific cases by computer, by way of the next result.

Proposition 5.15. Assuming $s>r / 2$, suppose there is some $\left(\Gamma^{\prime}, \Gamma, \Gamma^{\prime \prime}\right) \in \Sigma$ such that the series of curves of degree $2 r+5$ double at $\Gamma^{\prime}$ and containing $\Gamma$ has the expected dimension, and suppose some curve in this series has nodes at each point of $\Gamma^{\prime}$ and no other singularities. Then the curve class described in Conjecture 5.14 is a moving curve.

Proof. Consider the open subset

$$
\Sigma^{\circ}=\left\{\left(\Gamma^{\prime}, \Gamma, \Gamma^{\prime \prime}\right): h^{0}\left(\mathscr{I}_{\left(\Gamma^{\prime}\right)^{2} \cup \Gamma}(2 r+5)\right)=2 s-r\right\} \subset \Sigma .
$$

By hypothesis, it is nonempty, and since $\Sigma$ is irreducible it is dense and irreducible. Over $\Sigma^{\circ}$ there is a vector bundle whose fiber over $\left(\Gamma^{\prime}, \Gamma, \Gamma^{\prime \prime}\right)$ is $H^{0}\left(\mathscr{I}_{\left(\Gamma^{\prime}\right)^{2} \cup \Gamma}(2 r+5)\right)$, so the projective bundle

$$
\Xi=\left\{\left(\Gamma^{\prime}, \Gamma, \Gamma^{\prime \prime}, C\right):\left(\Gamma^{\prime}, \Gamma, \Gamma^{\prime \prime}\right) \in \Sigma^{\circ}, C \in \mathbb{P} H^{0}\left(\mathscr{I}_{\left(\Gamma^{\prime}\right)^{2} \cup \Gamma}(2 r+5)\right)\right\}
$$

is irreducible. In $\Xi$ there is an open subset where $C$ has no unprescribed singularities. Our assumption shows this subset is dense, and thus dominates $\mathscr{H}_{n}$.

Strictly speaking, we have only proved the proposition over $\mathbb{C}$, as our proof of the irreducibility of $\Sigma$ was over $\mathbb{C}$; we suspect there aren't any problems in characteristic $p$, where we actually need the proposition. Since we are merely gathering evidence, we won't worry about this. 




Figure 5.3: Macaulay2 script for verifying Conjecture 5.14 on the existence of moving curve classes. 
To utilize the proposition with Macaulay2, we take the following approach. Choose $r+2$ random $\mathbb{F}_{p}$-points $\Gamma^{\prime \prime}$ lying on $L$, and choose $n$ random $\mathbb{F}_{p}$-points $\Gamma$ in $\mathbb{P}_{\mathbb{F}_{p}}^{2}$. Choose a random pencil $\mathscr{D}$ of $(r+3)$-ics containing $\Gamma \cup \Gamma^{\prime \prime}$, and let $\Gamma^{\prime}$ be the residual base locus. Then verify that $H^{0}\left(\mathscr{I}_{\left(\Gamma^{\prime}\right)^{2} \cup \Gamma}(2 r+5)\right)$ has the expected dimension, take a random curve in this series, and check that it has only the prescribed singularities. The script in Figure 5.3 carries out this procedure for $n=32$; a successful output consists of two copies of "True," one for the expected dimension check and one for a check of the singularities of a random curve. This script runs very quickly, and can be used to provide evidence for the conjecture on moving curves for many $n$. Checking interpolation for the corresponding vector bundle provides a much larger computational bottleneck.

\subsection{A minimality property of Steiner bundles}

In Remark 5.1 we discussed three different natural sounding statements which could uniformly describe the effective cone of $\mathscr{H}_{n}$. The first and simplest such statement, Possibility 1, claims that for each $n$ one of the four different constructions of moving curves (three of which we know actually work, and one of which is conjectured) gives the edge of the moving cone.

Proposition 5.16. If Possibility 1 is true, then there exist $n$ such that the nontrivial edge of the effective cone of $\mathscr{H}_{n}$ is not spanned by a divisor $D_{E}$, where $E$ is a vector bundle on $\mathbb{P}^{2}$ having interpolation for $n$ points. In particular, this is true whenever

$$
\frac{s}{r} \in\left\{\alpha: \frac{3}{5}<\alpha<\frac{1}{\varphi}\right\} \backslash \Phi_{2}
$$

While the proposition itself may be of limited interest since it derives a conclusion from a 
statement we have no evidence for, it is a consequence of an interesting minimality property of Steiner bundles.

Recall that for every $n=r(r+1) / 2+s$ with $0 \leq s \leq r$ there is a moving curve $\gamma$ on $\mathscr{H}_{n}$ given by allowing $n$ points to move in a linear pencil on a smooth curve of degree $r$. We have

$$
\gamma \cdot H=r \quad \gamma \cdot \Delta=2\left(r^{2}-r+s\right)
$$

If $E$ is any vector bundle satisfying interpolation for $n$ points, then the associated divisor

$$
D_{E}=c_{1}(E) H-\frac{\mathrm{rk} E}{2} \Delta
$$

must satisfy $\gamma \cdot D_{E} \geq 0$, and so

$$
\mu(E) \geq \frac{r^{2}-r+s}{r}
$$

When equality holds in this inequality, the divisor $D_{E}$ spans an edge of the effective cone. We have seen that if $s / r \in \Phi_{2}$, then there are twists of Steiner bundles achieving equality. Our main result in this section provides a converse: if equality holds, then the bundle is the appropriate twist of a Steiner bundle.

Theorem 5.17. Let $E$ be a vector bundle with interpolation for $n$ points, and assume

$$
\mu(E)=\frac{r^{2}-r+s}{r}
$$

Then $E$ admits a resolution of the form

$$
0 \rightarrow \mathcal{O}_{\mathbb{P}^{2}}(r-2)^{s k} \rightarrow \mathcal{O}_{\mathbb{P}^{2}}(r-1)^{(s+r) k} \rightarrow E \rightarrow 0
$$

for some $k \in \mathbb{Q}$. 
In particular, no such bundle exists unless $s / r \in \Phi_{2}$. Indeed, if $E$ has a resolution of the specified form then it must be semistable by Proposition 4.5, and thus $s / r \in \Phi_{2}$ by Theorem 1.3. Proposition 5.16 follows by noting that Possibility 1 predicts the slope of the extremal edge of the effective cone is $\left(r^{2}-r+s\right) / r$ in case $3 / 5<s / r<\varphi^{-1}$.

The case where $s=0$ is easier than the general case, but treating it uniformly with the other cases would complicate things slightly. We leave the details of this case to the reader, and focus on the much more important cases where $0<s \leq r$.

To prove the theorem, we start by noting that any vector bundle $E$ on $\mathbb{P}^{2}$ admits a resolution of the form

$$
0 \rightarrow A \rightarrow B \rightarrow E \rightarrow 0
$$

where $A$ and $B$ are both direct sums of line bundles. We recall the argument from [3]. Indeed, since $E$ is locally free the graded $\mathbb{C}[x, y, z]$-module $H^{0}(E(*))$ is finitely generated. It is then easy to find a split bundle $B$ and a surjection $B \rightarrow E \rightarrow 0$ such that the maps $H^{0}(B(m)) \rightarrow H^{0}(E(m))$ are surjective for every $m$. The kernel $A$ of this surjection is then locally free, and Horrocks' splitting criterion [16] implies $A$ is split.

We may further assume that the resolution

$$
0 \rightarrow A \stackrel{M}{\rightarrow} B \rightarrow E \rightarrow 0
$$

is minimal, in the sense that all entries of the matrix $M$ lie in the irrelevant ideal $(x, y, z) \subset$ $\mathbb{C}[x, y, z]$. Observe that $h^{0}(E)$ depends only on the bundles $A$ and $B$, and not on $M$ (so long as $M$ is injective). It follows that if $E$ has interpolation for $n$ points, then the bundle $E^{\prime}$ corresponding to a general matrix $M^{\prime}: A \rightarrow B$ also has interpolation for $n$ points. It also has the same slope as $E$. 
If $A$ and $B$ have a common summand $\mathcal{O}_{\mathbb{P}^{2}}(a)$ then the resolution

$$
0 \rightarrow A \stackrel{M^{\prime}}{\rightarrow} B \rightarrow E^{\prime} \rightarrow 0
$$

fails to be minimal since $M^{\prime}$ will have some nonzero scalar entries. It is then possible to cancel some common factors and arrive at a resolution

$$
0 \rightarrow A^{\prime} \rightarrow B^{\prime} \rightarrow E^{\prime} \rightarrow 0
$$

where $A^{\prime}$ and $B^{\prime}$ have no common summand. If in fact $E^{\prime}$ admits a resolution of the form

$$
0 \rightarrow \mathcal{O}_{\mathbb{P}^{2}}(r-2)^{s k} \rightarrow \mathcal{O}_{\mathbb{P}^{2}}(r-1)^{(s+r) k} \rightarrow E^{\prime} \rightarrow 0
$$

for some $k \in \mathbb{Q}$, then it follows that $E$ does as well. For in this case, $E$ admits a minimal resolution of the form

$$
0 \rightarrow \mathcal{O}_{\mathbb{P}^{2}}(r-2)^{s k} \oplus C \stackrel{M}{\rightarrow} \mathcal{O}_{\mathbb{P}^{2}}(r-1)^{(s+r) k} \oplus C \rightarrow E \rightarrow 0
$$

for some split vector bundle $C$. If $\mathcal{O}_{\mathbb{P}^{2}}(a)$ is the largest twist of a line bundle appearing in $C$ and $a \geq r-1$, then by the minimality hypothesis this summand of $C$ must be in the kernel of $M$, contradicting that $M$ is injective. Similarly, if $\mathcal{O}_{\mathbb{P}^{2}}(b)$ is the smallest twist of a line bundle appearing in $C$ and $b \leq r-2$ then by minimality $E$ must have a direct summand isomorphic to $\mathcal{O}_{\mathbb{P}^{2}}(b)$. But then clearly $E$ does not satisfy interpolation for $n$ points, as $h^{0}\left(\mathcal{O}_{\mathbb{P}^{2}}(b)\right)<n$. It follows that $C=0$, and $E$ has the required resolution.

Thus, in order to prove the theorem we may assume that $E$ admits a resolution

$$
0 \rightarrow A \rightarrow B \rightarrow E \rightarrow 0
$$

by split bundles with no common factor. 
In order to impose restrictions on the resolution of an interpolation bundle with minimal slope, we isolate a numerical property of vector bundles satisfying interpolation (for $n$ points, as always in this section). For a split vector bundle $A$ and an integer $m$, we denote by $A_{m}$ the subbundle spanned by the summands $\mathcal{O}(a)$ having $a \geq m$, and we call it the $m$ th truncation of $A$. We further define a function $\nu$ on split vector bundles by

$$
\nu(A)=h^{1}\left(A \otimes \mathscr{I}_{\Gamma}\right)
$$

where $\Gamma$ is a general union of $n$ points.

To make things slightly less messy, it will be useful to be able to work with formal direct sums of line bundles with positive rational exponents instead of integer exponents. We will call such an object a $\mathbb{Q}$-split vector bundle. We extend the functions $c_{1}$, rk, $\nu$, and truncation $\mathbb{Q}$-linearly to the space of $\mathbb{Q}$-split bundles. Clearly every $\mathbb{Q}$-split bundle has some power which is an honest split bundle.

We now say that a pair $(A, B)$ of $\mathbb{Q}$-split vector bundles has numerically interpolative cokernel (for $n$ points) or is an NIC pair if the following conditions are satisfied.

1. $A$ and $B$ are $\mathbb{Q}$-linear sums of line bundles $\mathcal{O}(a)$ with $a \leq r-1$, and no summand of $A$ appears in $B$,

2. $\nu(A)=\nu(B)$,

3. for every integer $m$, we have $\nu\left(A_{m}\right) \leq \nu\left(B_{m}\right)$, and

4. if we define the slope $\mu(A, B)$ by

$$
\mu(A, B)=\frac{c_{1}(B)-c_{1}(A)}{\operatorname{rk} B-\operatorname{rk} A}
$$

then $\mu(A, B)$ is finite and $\mu(A, B)>r-1$. 
(In fact, assuming $(A, B)$ is nonzero, condition (4) can be seen to follow from the first three conditions, but we will not bother ourselves with the proof). Observe that every NIC pair of $\mathbb{Q}$-split bundles gives rise to an NIC pair of split bundles by taking sufficiently divisible powers.

Lemma 5.18. If a nonzero vector bundle E satisfies interpolation and has a resolution

$$
0 \rightarrow A \rightarrow B \rightarrow E \rightarrow 0
$$

by split bundles with no common factor, then $(A, B)$ is an NIC pair, and $\mu(A, B)=\mu(E)$.

Proof. Let $\Gamma$ be a general collection of $n$ points. Since $E$ satisfies interpolation, we find that the map $H^{0}\left(A \otimes \mathscr{I}_{\Gamma}\right) \rightarrow H^{0}\left(B \otimes \mathscr{I}_{\Gamma}\right)$ is an isomorphism. If we write $A$ as a sum of $\mathcal{O}\left(a_{i}\right)$ with $a_{1} \leq \cdots \leq a_{\alpha}$ and $B$ as a sum of $\mathcal{O}\left(b_{j}\right)$ with $b_{1} \leq \cdots \leq b_{\beta}$, then since we have an injection $A \rightarrow B$ we find $a_{i}<b_{\beta-\alpha+i}$ for $1 \leq i \leq \alpha$. Thus $h^{0}\left(\mathcal{O}\left(a_{i}\right) \otimes \mathscr{I}_{\Gamma}\right) \leq h^{0}\left(\mathcal{O}\left(b_{\beta-\alpha+i}\right) \otimes \mathscr{I}_{\Gamma}\right)$ for $1 \leq i \leq \alpha$, with equality if and only if all the $b_{\beta-\alpha+i}$ are no larger than $r-1$, and we conclude $h^{0}\left(A \otimes \mathscr{I}_{\Gamma}\right) \leq h^{0}\left(B \otimes \mathscr{I}_{\Gamma}\right)$, with equality if and only if $h^{0}\left(A \otimes \mathscr{I}_{\Gamma}\right)=h^{0}\left(B \otimes \mathscr{I}_{\Gamma}\right)=0$. Since equality must hold, we find that the summands in $A$ and $B$ are all of the form $\mathcal{O}(a)$ with $a \leq r-1$, confirming property (1).

We next claim that the map $H^{1}\left(A \otimes \mathscr{I}_{\Gamma}\right) \rightarrow H^{1}\left(B \otimes \mathscr{I}_{\Gamma}\right)$ is an isomorphism, so that 
$\nu(A)=\nu(B)$. It is clearly injective. From the diagram

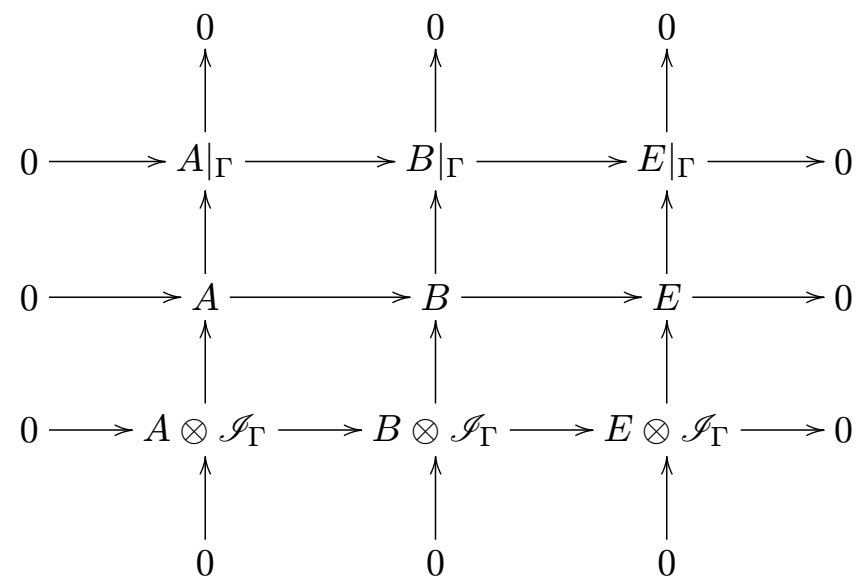

we obtain the commuting diagram

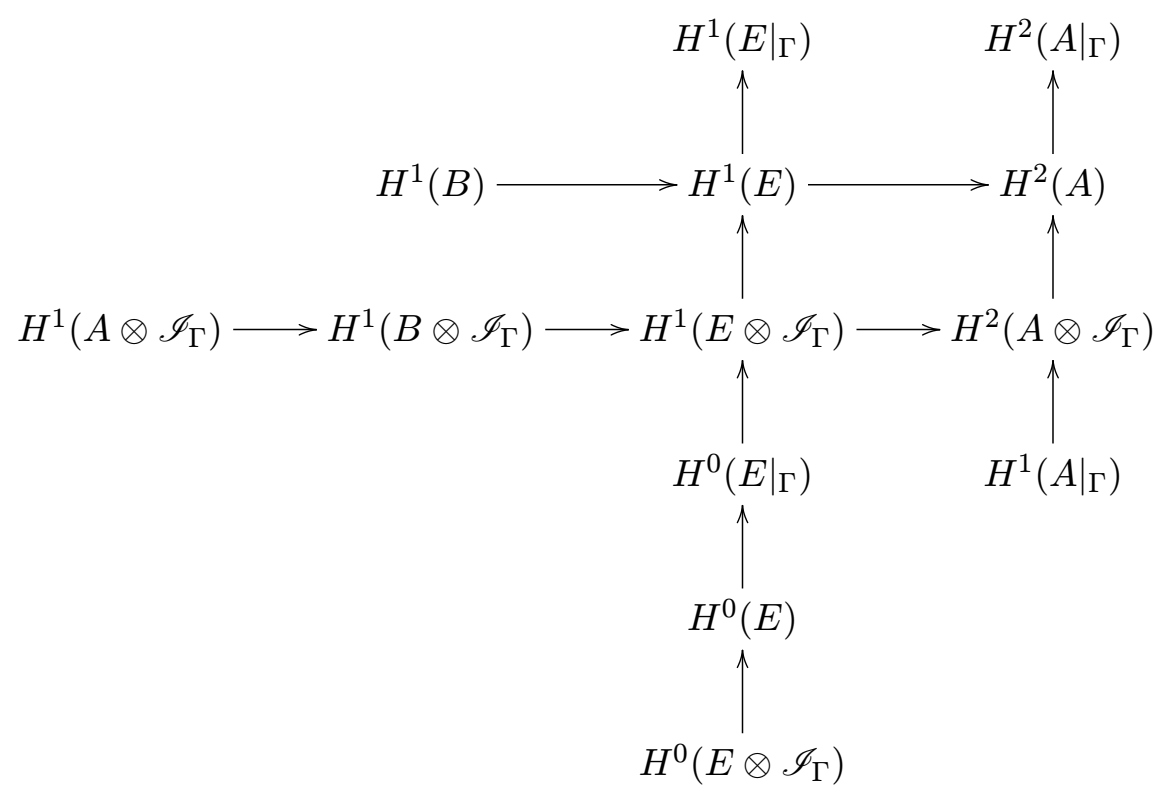

with exact rows and columns. Now $H^{1}\left(\left.E\right|_{\Gamma}\right), H^{2}\left(\left.A\right|_{\Gamma}\right), H^{1}(B), H^{1}\left(\left.A\right|_{\Gamma}\right)$, and $H^{0}\left(E \otimes \mathscr{I}_{\Gamma}\right)$ all vanish. Furthermore, since $E$ satisfies interpolation $H^{0}(E) \rightarrow H^{0}\left(\left.E\right|_{\Gamma}\right)$ is actually an isomorphism. It follows that the maps $H^{1}\left(E \otimes \mathscr{I}_{\Gamma}\right) \rightarrow H^{1}(E)$ and $H^{2}\left(A \otimes \mathscr{I}_{\Gamma}\right) \rightarrow H^{2}(A)$ are both isomorphisms, and since $H^{1}(E) \rightarrow H^{2}(A)$ is injective we find $H^{1}\left(E \otimes \mathscr{I}_{\Gamma}\right) \rightarrow$ 
$H^{2}\left(A \otimes \mathscr{I}_{\Gamma}\right)$ is injective. We conclude that $H^{1}\left(A \otimes \mathscr{I}_{\Gamma}\right) \rightarrow H^{1}\left(B \otimes \mathscr{I}_{\Gamma}\right)$ is surjective, hence an isomorphism, and property (2) holds.

Since $H^{1}\left(A \otimes \mathscr{I}_{\Gamma}\right) \rightarrow H^{1}\left(B \otimes \mathscr{I}_{\Gamma}\right)$ is an isomorphism, it follows that the induced maps

$$
H^{1}\left(A_{m} \otimes \mathscr{I}_{\Gamma}\right) \rightarrow H^{1}\left(B_{m} \otimes \mathscr{I}_{\Gamma}\right)
$$

are injective for every $m$, and thus $\nu\left(A_{m}\right) \leq \nu\left(B_{m}\right)$, verifying property (3).

Finally, we remark that $\mu(E)=\mu(A, B)$, and clearly $\mu(E)>r-1$, so property (4) holds. Thus $(A, B)$ has numerically interpolative cokernel.

Since vector bundles satisfying interpolation give rise to NIC pairs having the same slope, the next result implies the main theorem from this section.

Proposition 5.19. Let $(A, B)$ be an NIC pair of $\mathbb{Q}$-split bundles. Then

$$
\mu(A, B) \geq \frac{r^{2}-r+s}{r}
$$

with equality if and only if $(A, B)$ is of the form $\left(\mathcal{O}(r-2)^{s k}, \mathcal{O}(r-1)^{(s+r) k}\right)$ for some $k \in \mathbb{Q}$.

The main idea is to show that any NIC pair other than $\left(\mathcal{O}(r-2)^{s k}, \mathcal{O}(r-1)^{(s+r) k}\right)$ gives rise to a new NIC pair having strictly smaller slope, and that by performing such an operation finitely many times we can arrive at a pair of the form $\left(\mathcal{O}(r-2)^{s k}, \mathcal{O}(r-1)^{(s+r) k}\right)$.

Proof. Let $a$ be the smallest twist occurring as a summand of $A$, and let $b$ be the smallest twist occurring as a summand of $B$, so that we can write

$$
A=\mathcal{O}(a)^{\alpha} \oplus A_{a+1} \quad B=\mathcal{O}(b)^{\beta} \oplus B_{b+1} .
$$

We observe that the NIC-pair hypothesis implies $a<b$. For if $b<a$ we would have

$$
\nu\left(B_{a}\right)<\nu(B)=\nu(A)=\nu\left(A_{a}\right),
$$


contradicting $\nu\left(A_{a}\right) \leq \nu\left(B_{a}\right)$; furthermore $a=b$ is excluded by the minimality hypothesis. We use induction on $-(a+b)$.

We have $a+b \leq 2 r-3$, so the base case occurs when $a=r-2$ and $b=r-1$. Thus

$$
A=\mathcal{O}(r-2)^{\alpha} \quad B=\mathcal{O}(r-1)^{\beta}
$$

The hypothesis $\nu(A)=\nu(B)$ easily shows that we have $\alpha=s k$ and $\beta=(s+r) k$ for some $k \in \mathbb{Q}$, and we find $\mu(A, B)=\left(r^{2}-r+s\right) / r$.

Given an NIC-pair $(A, B)$ with $a+b<2 r-3$, we will now construct an NIC-pair $(C, D)$ such that $\mu(C, D) \leq \mu(A, B)$ and such that the corresponding sum $c+d$ of the smallest twists in $C$ and $D$ satisfies $c+d>a+b$. Furthermore, we will show that either the inequality $\mu(C, D) \leq \mu(A, B)$ is strict or $c \leq-2$, in which case the inequality will become strict at a later step. Our construction takes one of three forms, depending on the structure of $A$ and $B$.

Case 1: $b=r-1$. In this case we may write

$$
A=\mathcal{O}(a)^{\alpha} \oplus A_{a+1} \quad B=\mathcal{O}(r-1)^{\beta},
$$

and we have $r-a-2>0$. Put

$$
A^{\prime}=\mathcal{O}(a+1)^{\alpha+\frac{\alpha}{r-a-2}} \oplus A_{a+1} \quad B^{\prime}=\mathcal{O}(r-1)^{\beta+\frac{\alpha}{r-a-2}}
$$

and observe that $\mu\left(A^{\prime}, B^{\prime}\right)=\mu(A, B)$. Furthermore, notice that

$$
\nu\left(A^{\prime}\right)=\nu(A)+\frac{\alpha}{r-a-2} \nu(\mathcal{O}(a+1))+\alpha(\nu(\mathcal{O}(a+1))-\nu(\mathcal{O}(a)))
$$

and

$$
\nu\left(B^{\prime}\right)=\nu(B)+\frac{\alpha}{r-a-2} \nu(\mathcal{O}(r-1))
$$


Thus

$$
\nu\left(A^{\prime}\right)-\nu\left(B^{\prime}\right)=\frac{\alpha}{r-a-2}(\nu(\mathcal{O}(a+1))-\nu(\mathcal{O}(r-1)))+\alpha(\nu(\mathcal{O}(a+1))-\nu(\mathcal{O}(a))) .
$$

In case $a<-1$, this expression is clearly positive since then $\nu(\mathcal{O}(a))=\nu(\mathcal{O}(a+1))$. On the other hand for $a \geq-1$ we can use the formula

$$
\nu(\mathcal{O}(a))=\frac{r(r+1)}{2}+s-\left(\begin{array}{c}
a+2 \\
2
\end{array}\right)
$$

to find

$$
\nu\left(A^{\prime}\right)-\nu\left(B^{\prime}\right)=\frac{1}{2} \alpha(r-a-2)(r-a-1),
$$

which is also positive. We can then choose some positive rational number $\gamma$ such that the pair

$$
C=A^{\prime} \quad D=B^{\prime} \oplus \mathcal{O}(r-1)^{\gamma}
$$

satisfies $\nu(C)=\nu(D)$. Due to the form of $D$, we also conclude $\nu\left(C_{m}\right) \leq \nu\left(D_{m}\right)$ for every $m$. Now $\mu(C, D)$ is a weighted average of $r-1$ and $\mu\left(A^{\prime}, B^{\prime}\right)$, so since $\mu\left(A^{\prime}, B^{\prime}\right)>r-1$ we find

$$
r-1<\mu(C, D)<\mu\left(A^{\prime}, B^{\prime}\right)=\mu(A, B) .
$$

Notice that the sum $c+d$ of the smallest twists in $C$ and $D$ satisfies $c+d>a+b$. If $C$ and $D$ have any common summands we can cancel them without affecting the numerics, so we conclude that $(C, D)$ is an NIC pair with the required properties.

Case 2: $b<r-1$ and $\alpha \leq \beta$. Here we write

$$
A=\mathcal{O}(a)^{\alpha} \oplus A_{a+1} \quad B=\mathcal{O}(b)^{\beta} \oplus B_{b+1} .
$$

We first modify this pair by putting

$$
A^{\prime}=\mathcal{O}(a+1)^{\alpha} \oplus A_{a+1} \quad B^{\prime}=\mathcal{O}(b)^{\beta-\alpha} \oplus \mathcal{O}(b+1)^{\alpha} \oplus B_{b+1}
$$


Notice that $\mu\left(A^{\prime}, B^{\prime}\right)=\mu(A, B)$. Furthermore, we have $\nu\left(A^{\prime}\right) \geq \nu\left(B^{\prime}\right)$, since $a<b$ implies that

$$
\nu(A)-\nu\left(A^{\prime}\right) \leq \nu(B)-\nu\left(B^{\prime}\right) .
$$

The inequality $\nu\left(A^{\prime}\right) \geq \nu\left(B^{\prime}\right)$ is strict unless $b \leq-2$.

In case $b \leq-2$, we claim that the twist $\mathcal{O}(a+1)$ does not occur in $B^{\prime}$. If $\alpha=\beta$, then this follows from the fact that $a<b$. So instead assume $\alpha<\beta$. Then $B^{\prime}$ still has a twist of index $b$. We cannot have $b=a+1$, for then it follows that $A_{a+1}=A_{a+2}$ and $\nu\left(A_{a+2}\right)>\nu\left(B_{a+2}\right)$ since $\alpha<\beta$. Thus $a+1<b$, and $\mathcal{O}(a+1)$ does not occur in $B^{\prime}$.

We may now choose a nonnegative rational number $\gamma$ so that if

$$
C=A^{\prime} \quad D=B^{\prime} \oplus \mathcal{O}(r-1)^{\gamma}
$$

then $\nu(C)=\nu(D)$. As in the previous case, we find $r-1<\mu(C, D) \leq \mu(A, B)$, and the last inequality is necessarily strict unless the number $c$ corresponding to the smallest twist in $C$ has $c \leq-2$. By construction we have $\nu\left(C_{m}\right) \leq \nu\left(D_{m}\right)$ whenever $m \leq b$. For $m \geq b+1$, we observe that $\nu\left(D_{m}\right) \geq \nu\left(B_{m}\right)$ whereas $\nu\left(C_{m}\right)=\nu\left(A_{m}\right)$, so also $\nu\left(C_{m}\right) \leq \nu\left(D_{m}\right)$ in this case. Cancelling common summands if necessary, $(C, D)$ has the required properties.

Case 3: $b<r-1$ and $\alpha>\beta$. Similarly to the previous case, put

$$
A^{\prime}=\mathcal{O}(a)^{\beta} \oplus \mathcal{O}(a+1)^{\alpha-\beta} \oplus A_{a+1} \quad B^{\prime}=\mathcal{O}(b+1)^{\beta} \oplus B_{b+1},
$$

then choose a nonnegative number $\gamma$ so that the bundles

$$
C=A^{\prime} \quad D=B^{\prime} \oplus \mathcal{O}(r-1)^{\gamma}
$$

satisfy $\nu(C)=\nu(D)$. The full details are essentially the same. 


\section{Appendix A}

\section{Table of cones of effective divisors}

\section{and moving curves on $\mathscr{H}_{n}$}

Write $n=r(r+1) / 2+s$. For small values of $n$, we know the nontrivial extremal moving curve class on $\mathscr{H}_{n}$ is given by allowing $n$ points to move in a linear pencil on the normalization $\tilde{C}_{n}$ of a curve $C_{n}$ in $\mathbb{P}^{2}$ of some degree having some number $\delta\left(C_{n}\right)$ of nodes and no other singularities. Multiples of $D_{n}$ can be represented as loci where interpolation fails for a vector bundle $E_{n}$ on $\mathbb{P}^{2}$ having interpolation for $n$ points. If $D_{n}=a H-\frac{b}{2} \Delta$, we denote by $\mu\left(D_{n}\right)=a / b$ the slope of the divisor class $D_{n}$. Note that $\mu\left(D_{n}\right)=\mu\left(E_{n}\right)$, and by Proposition 3.3 we have $\mu\left(D_{n}\right) \leq \mu\left(D_{n+1}\right)$ for all $n$.

In the following table, data for indices $n$ with no annotation follows from Theorem 1.8. We mark $n$ with various symbols based on the status of establishing the corresponding data.

- *: For these indices (e.g. $n=17$ ), the described curve class is in fact a moving curve class by Theorem 5.5, and interpolation for $E_{n}$ is checked over a finite field with Macaulay, as described in Section 5.4. 
- **: The described curve class is moving by Theorem 5.5, but interpolation has not been checked for $E_{n}$.

- $\dagger$ : The moving curve class and interpolation for $E_{n}$ were checked over a finite field with Macaulay.

- $\ddagger$ : The moving curve class was checked over a finite field with Macaulay. Interpolation for $E_{n}$ takes too long to check with an ordinary computer, and remains completely open.

Table A.1: Effective and moving cones of $\mathscr{H}_{n}$

\begin{tabular}{ccccccc}
\hline$n$ & $r$ & $s$ & $\operatorname{deg} C_{n}$ & $\delta\left(C_{n}\right)$ & $E_{n}$ & $\mu\left(D_{n}\right)$ \\
\hline 3 & 2 & 0 & 2 & 0 & $\mathcal{O}(1)$ & 1 \\
4 & 2 & 1 & 2 & 0 & $\operatorname{coker}\left(\mathcal{O} \rightarrow \mathcal{O}(1)^{3}\right)$ & $3 / 2$ \\
5 & 2 & 2 & 2 & 0 & $\operatorname{coker}\left(\mathcal{O}^{2} \rightarrow \mathcal{O}(1)^{4}\right)$ & 2 \\
6 & 3 & 0 & 3 & 0 & $\mathcal{O}(2)$ & 2 \\
7 & 3 & 1 & 5 & 0 & $\operatorname{ker}\left(\mathcal{O}(3)^{8} \rightarrow \mathcal{O}(4)^{3}\right)$ & $12 / 5$ \\
8 & 3 & 2 & 3 & 0 & $\operatorname{coker}\left(\mathcal{O}(1)^{2} \rightarrow \mathcal{O}(2)^{5}\right)$ & $8 / 3$ \\
9 & 3 & 3 & 3 & 0 & $\operatorname{coker}\left(\mathcal{O}(1)^{3} \rightarrow \mathcal{O}(2)^{6}\right)$ & 3 \\
10 & 4 & 0 & 4 & 0 & $\operatorname{O}(3)$ & 3 \\
11 & 4 & 1 & 6 & 0 & $\operatorname{ker}\left(\mathcal{O}(4)^{10} \rightarrow \mathcal{O}(5)^{4}\right)$ & $10 / 3$ \\
12 & 4 & 2 & 4 & 0 & $\operatorname{coker}\left(\mathcal{O}(2)^{2} \rightarrow \mathcal{O}(3)^{6}\right)$ & $7 / 2$ \\
13 & 4 & 3 & 4 & 0 & $\operatorname{coker}\left(\mathcal{O}(2)^{3} \rightarrow \mathcal{O}(3)^{7}\right)$ & $15 / 4$ \\
14 & 4 & 4 & 4 & 0 & $\operatorname{coker}\left(\mathcal{O}(2)^{4} \rightarrow \mathcal{O}(3)^{8}\right)$ & 4 \\
15 & 5 & 0 & 5 & 0 & $\mathcal{O}(4)$ & 4 \\
16 & 5 & 1 & 7 & 0 & $\operatorname{ker}\left(\mathcal{O}(5)^{12} \rightarrow \mathcal{O}(6)^{5}\right)$ & $30 / 7$ \\
$17^{*}$ & 5 & 2 & 9 & 4 & $\operatorname{coker}\left(\mathcal{O}(2)^{2} \rightarrow \mathcal{O}(4)^{11}\right)$ & $40 / 9$ \\
18 & 5 & 3 & 5 & 0 & $\operatorname{coker}\left(\mathcal{O}(3)^{3} \rightarrow \mathcal{O}(4)^{8}\right)$ & $23 / 5$ \\
19 & 5 & 4 & 5 & 0 & $\operatorname{coker}\left(\mathcal{O}(3)^{4} \rightarrow \mathcal{O}(4)^{9}\right)$ & $24 / 5$ \\
20 & 5 & 5 & 5 & 0 & $\operatorname{coker}\left(\mathcal{O}(3)^{5} \rightarrow \mathcal{O}(4)^{10}\right)$ & 5 \\
21 & 6 & 0 & 6 & 0 & $\mathcal{O}(5)$ & 5 \\
22 & 6 & 1 & 8 & 0 & $\operatorname{ker}\left(\mathcal{O}(6)^{14} \rightarrow \mathcal{O}(7)^{6}\right)$ & $21 / 4$ \\
23 & 6 & 2 & 8 & 0 & $\operatorname{ker}\left(\mathcal{O}(6)^{13} \rightarrow \mathcal{O}(7)^{5}\right)$ & $43 / 8$ \\
24 & 6 & 3 & 6 & 0 & $\operatorname{coker}\left(\mathcal{O}(4)^{3} \rightarrow \mathcal{O}(5)^{9}\right)$ & $11 / 2$ \\
25 & 6 & 4 & 6 & 0 & $\operatorname{coker}\left(\mathcal{O}(4)^{4} \rightarrow \mathcal{O}(5)^{10}\right)$ & $17 / 3$ \\
\hline & & & & & $\operatorname{continued}$ on & next page
\end{tabular}


continued from previous page

\begin{tabular}{|c|c|c|c|c|c|c|}
\hline$n$ & $r$ & $s$ & $\operatorname{deg} C_{n}$ & $\delta\left(C_{n}\right)$ & $E_{n}$ & $\mu\left(D_{n}\right)$ \\
\hline 26 & 6 & 5 & 6 & 0 & $\operatorname{coker}\left(\mathcal{O}(4)^{5} \rightarrow \mathcal{O}(5)^{11}\right)$ & $35 / 6$ \\
\hline 27 & 6 & 6 & 6 & 0 & $\operatorname{coker}\left(\mathcal{O}(4)^{6} \rightarrow \mathcal{O}(5)^{12}\right)$ & 6 \\
\hline 28 & 7 & 0 & 7 & 0 & $\mathcal{O}(6)$ & 6 \\
\hline 29 & 7 & 1 & 9 & 0 & $\operatorname{ker}\left(\mathcal{O}(7)^{16} \rightarrow \mathcal{O}(8)^{7}\right)$ & $56 / 9$ \\
\hline 30 & 7 & 2 & 9 & 0 & $\operatorname{ker}\left(\mathcal{O}(7)^{15} \rightarrow \mathcal{O}(8)^{6}\right)$ & $19 / 3$ \\
\hline $31^{*}$ & 7 & 3 & 13 & 12 & $\operatorname{coker}\left(\mathcal{O}(4)^{3} \rightarrow \mathcal{O}(6)^{16}\right)$ & $84 / 13$ \\
\hline $32^{\dagger}$ & 7 & 4 & 19 & 59 & $\operatorname{ker}\left(\mathcal{O}(7)^{23} \rightarrow \mathcal{O}(9)^{4}\right)$ & $125 / 19$ \\
\hline 33 & 7 & 5 & 7 & 0 & $\operatorname{coker}\left(\mathcal{O}(5)^{5} \rightarrow \mathcal{O}(6)^{12}\right)$ & $47 / 7$ \\
\hline 34 & 7 & 6 & 7 & 0 & $\operatorname{coker}\left(\mathcal{O}(5)^{6} \rightarrow \mathcal{O}(6)^{13}\right)$ & $48 / 7$ \\
\hline 35 & 7 & 7 & 7 & 0 & $\operatorname{coker}\left(\mathcal{O}(5)^{7} \rightarrow \mathcal{O}(6)^{14}\right)$ & 7 \\
\hline 36 & 8 & 0 & 8 & 0 & $\mathcal{O}(7)$ & 7 \\
\hline 37 & 8 & 1 & 10 & 0 & $\operatorname{ker}\left(\mathcal{O}(8)^{18} \rightarrow \mathcal{O}(9)^{8}\right)$ & $36 / 5$ \\
\hline 38 & 8 & 2 & 10 & 0 & $\operatorname{ker}\left(\mathcal{O}(8)^{17} \rightarrow \mathcal{O}(9)^{7}\right)$ & $73 / 10$ \\
\hline 39 & 8 & 3 & 10 & 0 & $\operatorname{ker}\left(\mathcal{O}(8)^{16} \rightarrow \mathcal{O}(9)^{6}\right)$ & $37 / 5$ \\
\hline 40 & 8 & 4 & 8 & 0 & $\operatorname{coker}\left(\mathcal{O}(6)^{4} \rightarrow \mathcal{O}(7)^{12}\right)$ & $15 / 2$ \\
\hline 41 & 8 & 5 & 8 & 0 & $\operatorname{coker}\left(\mathcal{O}(6)^{5} \rightarrow \mathcal{O}(7)^{13}\right)$ & $61 / 8$ \\
\hline 42 & 8 & 6 & 8 & 0 & $\operatorname{coker}\left(\mathcal{O}(6)^{6} \rightarrow \mathcal{O}(7)^{14}\right)$ & $31 / 4$ \\
\hline 43 & 8 & 7 & 8 & 0 & $\operatorname{coker}\left(\mathcal{O}(6)^{7} \rightarrow \mathcal{O}(7)^{15}\right)$ & $63 / 8$ \\
\hline 44 & 8 & 8 & 8 & 0 & $\operatorname{coker}\left(\mathcal{O}(6)^{8} \rightarrow \mathcal{O}(7)^{16}\right)$ & 8 \\
\hline 45 & 9 & 0 & 9 & 0 & $\mathcal{O}(8)$ & 8 \\
\hline 46 & 9 & 1 & 11 & 0 & $\operatorname{ker}\left(\mathcal{O}(9)^{20} \rightarrow \mathcal{O}(10)^{9}\right)$ & $90 / 11$ \\
\hline 47 & 9 & 2 & 11 & 0 & $\operatorname{ker}\left(\mathcal{O}(9)^{19} \rightarrow \mathcal{O}(10)^{8}\right)$ & $91 / 11$ \\
\hline 48 & 9 & 3 & 11 & 0 & $\operatorname{ker}\left(\mathcal{O}(9)^{18} \rightarrow \mathcal{O}(10)^{7}\right)$ & $92 / 11$ \\
\hline $49^{*}$ & 9 & 4 & 17 & 24 & $\operatorname{coker}\left(\mathcal{O}(6)^{4} \rightarrow \mathcal{O}(8)^{21}\right)$ & $144 / 17$ \\
\hline $50^{\dagger}$ & 9 & 5 & 23 & 83 & $\operatorname{ker}\left(\mathcal{O}(9)^{28} \rightarrow \mathcal{O}(11)^{5}\right)$ & $197 / 23$ \\
\hline 51 & 9 & 6 & 9 & 0 & $\operatorname{coker}\left(\mathcal{O}(7)^{6} \rightarrow \mathcal{O}(8)^{15}\right)$ & $26 / 3$ \\
\hline 52 & 9 & 7 & 9 & 0 & $\operatorname{coker}\left(\mathcal{O}(7)^{7} \rightarrow \mathcal{O}(8)^{16}\right)$ & $79 / 9$ \\
\hline 53 & 9 & 8 & 9 & 0 & $\operatorname{coker}\left(\mathcal{O}(7)^{8} \rightarrow \mathcal{O}(8)^{17}\right)$ & $80 / 9$ \\
\hline 54 & 9 & 9 & 9 & 0 & $\operatorname{coker}\left(\mathcal{O}(7)^{9} \rightarrow \mathcal{O}(8)^{18}\right)$ & 9 \\
\hline 55 & 10 & 0 & 10 & 0 & $\mathcal{O}(9)$ & 9 \\
\hline 56 & 10 & 1 & 12 & 0 & $\operatorname{ker}\left(\mathcal{O}(10)^{22} \rightarrow \mathcal{O}(11)^{10}\right)$ & $55 / 6$ \\
\hline 57 & 10 & 2 & 12 & 0 & $\operatorname{ker}\left(\mathcal{O}(10)^{21} \rightarrow \mathcal{O}(11)^{9}\right)$ & $37 / 4$ \\
\hline 58 & 10 & 3 & 12 & 0 & $\operatorname{ker}\left(\mathcal{O}(10)^{20} \rightarrow \mathcal{O}(11)^{8}\right)$ & $28 / 3$ \\
\hline $59^{*}$ & 10 & 4 & 19 & 32 & $\operatorname{coker}\left(\mathcal{O}(7)^{4} \rightarrow \mathcal{O}(9)^{23}\right)$ & $179 / 19$ \\
\hline 60 & 10 & 5 & 10 & 0 & $\operatorname{coker}\left(\mathcal{O}(8)^{5} \rightarrow \mathcal{O}(9)^{15}\right)$ & $19 / 2$ \\
\hline 61 & 10 & 6 & 10 & 0 & $\operatorname{coker}\left(\mathcal{O}(8)^{6} \rightarrow \mathcal{O}(9)^{16}\right)$ & $48 / 5$ \\
\hline 62 & 10 & 7 & 10 & 0 & $\operatorname{coker}\left(\mathcal{O}(8)^{7} \rightarrow \mathcal{O}(9)^{17}\right)$ & $97 / 10$ \\
\hline 63 & 10 & 8 & 10 & 0 & $\operatorname{coker}\left(\mathcal{O}(8)^{8} \rightarrow \mathcal{O}(9)^{18}\right)$ & $49 / 5$ \\
\hline 64 & 10 & 9 & 10 & 0 & $\operatorname{coker}\left(\mathcal{O}(8)^{9} \rightarrow \mathcal{O}(9)^{19}\right)$ & $99 / 10$ \\
\hline
\end{tabular}




\begin{tabular}{|c|c|c|c|c|c|c|}
\hline$n$ & $r$ & $s$ & $\operatorname{deg} C_{n}$ & $\delta\left(C_{n}\right)$ & $E_{n}$ & $\mu\left(D_{n}\right)$ \\
\hline 65 & 10 & 10 & 10 & 0 & $\operatorname{coker}\left(\mathcal{O}(8)^{10} \rightarrow \mathcal{O}(9)^{20}\right)$ & 10 \\
\hline 66 & 11 & 0 & 11 & 0 & $\mathcal{O}(10)$ & 10 \\
\hline 67 & 11 & 1 & 13 & 0 & $\operatorname{ker}\left(\mathcal{O}(11)^{24} \rightarrow \mathcal{O}(12)^{11}\right)$ & $132 / 13$ \\
\hline 68 & 11 & 2 & 13 & 0 & $\operatorname{ker}\left(\mathcal{O}(11)^{23} \rightarrow \mathcal{O}(12)^{10}\right)$ & $133 / 13$ \\
\hline 69 & 11 & 3 & 13 & 0 & $\operatorname{ker}\left(\mathcal{O}(11)^{22} \rightarrow \mathcal{O}(12)^{9}\right)$ & $134 / 13$ \\
\hline 70 & 11 & 4 & 13 & 0 & $\operatorname{ker}\left(\mathcal{O}(11)^{21} \rightarrow \mathcal{O}(12)^{8}\right)$ & $135 / 13$ \\
\hline $71^{*}$ & 11 & 5 & 21 & 40 & $\operatorname{coker}\left(\mathcal{O}(8)^{5} \rightarrow \mathcal{O}(10)^{26}\right)$ & $220 / 21$ \\
\hline $72^{\ddagger}$ & 11 & 6 & 27 & 111 & $\operatorname{ker}\left(\mathcal{O}(11)^{33} \rightarrow \mathcal{O}(13)^{6}\right)$ & $95 / 9$ \\
\hline 73 & 11 & 7 & 11 & 0 & $\operatorname{coker}\left(\mathcal{O}(9)^{7} \rightarrow \mathcal{O}(10)^{18}\right)$ & $117 / 11$ \\
\hline 74 & 11 & 8 & 11 & 0 & $\operatorname{coker}\left(\mathcal{O}(9)^{8} \rightarrow \mathcal{O}(10)^{19}\right)$ & $118 / 11$ \\
\hline 75 & 11 & 9 & 11 & 0 & $\operatorname{coker}\left(\mathcal{O}(9)^{9} \rightarrow \mathcal{O}(10)^{20}\right)$ & $119 / 11$ \\
\hline 76 & 11 & 10 & 11 & 0 & $\operatorname{coker}\left(\mathcal{O}(9)^{10} \rightarrow \mathcal{O}(10)^{21}\right)$ & $120 / 11$ \\
\hline 77 & 11 & 11 & 11 & 0 & $\operatorname{coker}\left(\mathcal{O}(9)^{11} \rightarrow \mathcal{O}(10)^{22}\right)$ & 11 \\
\hline 78 & 12 & 0 & 12 & 0 & $\mathcal{O}(11)$ & 11 \\
\hline 79 & 12 & 1 & 14 & 0 & $\operatorname{ker}\left(\mathcal{O}(12)^{26} \rightarrow \mathcal{O}(13)^{12}\right)$ & $78 / 7$ \\
\hline 80 & 12 & 2 & 14 & 0 & $\operatorname{ker}\left(\mathcal{O}(12)^{25} \rightarrow \mathcal{O}(13)^{11}\right)$ & $157 / 14$ \\
\hline 81 & 12 & 3 & 14 & 0 & $\operatorname{ker}\left(\mathcal{O}(12)^{24} \rightarrow \mathcal{O}(13)^{10}\right)$ & $79 / 7$ \\
\hline 82 & 12 & 4 & 14 & 0 & $\operatorname{ker}\left(\mathcal{O}(12)^{23} \rightarrow \mathcal{O}(13)^{9}\right)$ & $159 / 14$ \\
\hline $83^{*}$ & 12 & 5 & 23 & 50 & $\operatorname{coker}\left(\mathcal{O}(9)^{5} \rightarrow \mathcal{O}(11)^{28}\right)$ & $263 / 23$ \\
\hline 84 & 12 & 6 & 12 & 0 & $\operatorname{coker}\left(\mathcal{O}(10)^{6} \rightarrow \mathcal{O}(11)^{18}\right)$ & $23 / 2$ \\
\hline $85^{\ddagger}$ & 12 & 7 & 29 & 126 & $\operatorname{ker}\left(\mathcal{O}(12)^{35} \rightarrow \mathcal{O}(14)^{6}\right)$ & $336 / 29$ \\
\hline 86 & 12 & 8 & 12 & 0 & $\operatorname{coker}\left(\mathcal{O}(10)^{8} \rightarrow \mathcal{O}(11)^{20}\right)$ & $35 / 3$ \\
\hline 87 & 12 & 9 & 12 & 0 & $\operatorname{coker}\left(\mathcal{O}(10)^{9} \rightarrow \mathcal{O}(11)^{21}\right)$ & $47 / 4$ \\
\hline 88 & 12 & 10 & 12 & 0 & $\operatorname{coker}\left(\mathcal{O}(10)^{10} \rightarrow \mathcal{O}(11)^{22}\right)$ & $71 / 6$ \\
\hline 89 & 12 & 11 & 12 & 0 & $\operatorname{coker}\left(\mathcal{O}(10)^{11} \rightarrow \mathcal{O}(11)^{23}\right)$ & $143 / 12$ \\
\hline 90 & 12 & 12 & 12 & 0 & $\operatorname{coker}\left(\mathcal{O}(10)^{12} \rightarrow \mathcal{O}(11)^{24}\right)$ & 12 \\
\hline 91 & 13 & 0 & 13 & 0 & $\mathcal{O}(12)$ & 12 \\
\hline 92 & 13 & 1 & 15 & 0 & $\operatorname{ker}\left(\mathcal{O}(13)^{28} \rightarrow \mathcal{O}(14)^{13}\right)$ & $182 / 15$ \\
\hline 93 & 13 & 2 & 15 & 0 & $\operatorname{ker}\left(\mathcal{O}(13)^{27} \rightarrow \mathcal{O}(14)^{12}\right)$ & $61 / 5$ \\
\hline 94 & 13 & 3 & 15 & 0 & $\operatorname{ker}\left(\mathcal{O}(13)^{26} \rightarrow \mathcal{O}(14)^{11}\right)$ & $184 / 15$ \\
\hline 95 & 13 & 4 & 15 & 0 & $\operatorname{ker}\left(\mathcal{O}(13)^{25} \rightarrow \mathcal{O}(14)^{10}\right)$ & $37 / 3$ \\
\hline 96 & 13 & 5 & 15 & 0 & $\operatorname{ker}\left(\mathcal{O}(13)^{24} \rightarrow \mathcal{O}(14)^{9}\right)$ & $62 / 5$ \\
\hline $97^{*}$ & 13 & 6 & 25 & 60 & $\operatorname{coker}\left(\mathcal{O}(10)^{6} \rightarrow \mathcal{O}(12)^{31}\right)$ & $312 / 25$ \\
\hline $98^{\ddagger}$ & 13 & 7 & 31 & 143 & $\operatorname{ker}\left(\mathcal{O}(13)^{38} \rightarrow \mathcal{O}(15)^{7}\right)$ & $389 / 31$ \\
\hline 99 & 13 & 8 & 13 & 0 & $\operatorname{coker}\left(\mathcal{O}(11)^{8} \rightarrow \mathcal{O}(12)^{21}\right)$ & $164 / 13$ \\
\hline 100 & 13 & 9 & 13 & 0 & $\operatorname{coker}\left(\mathcal{O}(11)^{9} \rightarrow \mathcal{O}(12)^{22}\right)$ & $165 / 13$ \\
\hline 101 & 13 & 10 & 13 & 0 & $\operatorname{coker}\left(\mathcal{O}(11)^{10} \rightarrow \mathcal{O}(12)^{23}\right)$ & $166 / 13$ \\
\hline 102 & 13 & 11 & 13 & 0 & $\operatorname{coker}\left(\mathcal{O}(11)^{11} \rightarrow \mathcal{O}(12)^{24}\right)$ & $167 / 13$ \\
\hline 103 & 13 & 12 & 13 & 0 & $\operatorname{coker}\left(\mathcal{O}(11)^{12} \rightarrow \mathcal{O}(12)^{25}\right)$ & $168 / 13$ \\
\hline
\end{tabular}


continued from previous page

\begin{tabular}{|c|c|c|c|c|c|c|}
\hline$n$ & $r$ & $s$ & $\operatorname{deg} C_{n}$ & $\delta\left(C_{n}\right)$ & $E_{n}$ & $\mu\left(D_{n}\right)$ \\
\hline 104 & 13 & 13 & 13 & 0 & $\operatorname{coker}\left(\mathcal{O}(11)^{13} \rightarrow \mathcal{O}(12)^{26}\right)$ & 13 \\
\hline 105 & 14 & 0 & 14 & 0 & $\mathcal{O}(13)$ & 13 \\
\hline 106 & 14 & 1 & 16 & 0 & $\operatorname{ker}\left(\mathcal{O}(14)^{30} \rightarrow \mathcal{O}(15)^{14}\right)$ & $105 / 8$ \\
\hline 107 & 14 & 2 & 16 & 0 & $\operatorname{ker}\left(\mathcal{O}(14)^{29} \rightarrow \mathcal{O}(15)^{13}\right)$ & $211 / 16$ \\
\hline 108 & 14 & 3 & 16 & 0 & $\operatorname{ker}\left(\mathcal{O}(14)^{28} \rightarrow \mathcal{O}(15)^{12}\right)$ & $53 / 4$ \\
\hline 109 & 14 & 4 & 16 & 0 & $\operatorname{ker}\left(\mathcal{O}(14)^{27} \rightarrow \mathcal{O}(15)^{11}\right)$ & $213 / 16$ \\
\hline 110 & 14 & 5 & 16 & 0 & $\operatorname{ker}\left(\mathcal{O}(14)^{26} \rightarrow \mathcal{O}(15)^{10}\right)$ & $107 / 8$ \\
\hline $111^{*}$ & 14 & 6 & 27 & 72 & $\operatorname{coker}\left(\mathcal{O}(11)^{6} \rightarrow \mathcal{O}(13)^{33}\right)$ & $121 / 9$ \\
\hline 112 & 14 & 7 & 14 & 0 & $\operatorname{coker}\left(\mathcal{O}(12)^{7} \rightarrow \mathcal{O}(13)^{21}\right)$ & $27 / 2$ \\
\hline $113^{\ddagger}$ & 14 & 8 & 33 & 160 & $\operatorname{ker}\left(\mathcal{O}(14)^{40} \rightarrow \mathcal{O}(16)^{7}\right)$ & $448 / 33$ \\
\hline 114 & 14 & 9 & 14 & 0 & $\operatorname{coker}\left(\mathcal{O}(12)^{9} \rightarrow \mathcal{O}(13)^{23}\right)$ & $191 / 14$ \\
\hline 115 & 14 & 10 & 14 & 0 & $\operatorname{coker}\left(\mathcal{O}(12)^{10} \rightarrow \mathcal{O}(13)^{24}\right)$ & $96 / 7$ \\
\hline 116 & 14 & 11 & 14 & 0 & $\operatorname{coker}\left(\mathcal{O}(12)^{11} \rightarrow \mathcal{O}(13)^{25}\right)$ & $193 / 14$ \\
\hline 117 & 14 & 12 & 14 & 0 & $\operatorname{coker}\left(\mathcal{O}(12)^{12} \rightarrow \mathcal{O}(13)^{26}\right)$ & $97 / 7$ \\
\hline 118 & 14 & 13 & 14 & 0 & $\operatorname{coker}\left(\mathcal{O}(12)^{13} \rightarrow \mathcal{O}(13)^{27}\right)$ & $195 / 14$ \\
\hline 119 & 14 & 14 & 14 & 0 & $\operatorname{coker}\left(\mathcal{O}(12)^{14} \rightarrow \mathcal{O}(13)^{28}\right)$ & 14 \\
\hline 120 & 15 & 0 & 15 & 0 & $\mathcal{O}(14)$ & 14 \\
\hline 121 & 15 & 1 & 17 & 0 & $\operatorname{ker}\left(\mathcal{O}(15)^{32} \rightarrow \mathcal{O}(16)^{15}\right)$ & $240 / 17$ \\
\hline 122 & 15 & 2 & 17 & 0 & $\operatorname{ker}\left(\mathcal{O}(15)^{31} \rightarrow \mathcal{O}(16)^{14}\right)$ & $241 / 17$ \\
\hline 123 & 15 & 3 & 17 & 0 & $\operatorname{ker}\left(\mathcal{O}(15)^{30} \rightarrow \mathcal{O}(16)^{13}\right)$ & $242 / 17$ \\
\hline 124 & 15 & 4 & 17 & 0 & $\operatorname{ker}\left(\mathcal{O}(15)^{29} \rightarrow \mathcal{O}(16)^{12}\right)$ & $243 / 17$ \\
\hline 125 & 15 & 5 & 17 & 0 & $\operatorname{ker}\left(\mathcal{O}(15)^{28} \rightarrow \mathcal{O}(16)^{11}\right)$ & $244 / 17$ \\
\hline $126^{*}$ & 15 & 6 & 29 & 85 & $\operatorname{coker}\left(\mathcal{O}(12)^{6} \rightarrow \mathcal{O}(14)^{35}\right)$ & $418 / 29$ \\
\hline $127^{*}$ & 15 & 7 & 29 & 84 & $\operatorname{coker}\left(\mathcal{O}(12)^{7} \rightarrow \mathcal{O}(14)^{36}\right)$ & $420 / 29$ \\
\hline $128^{\ddagger}$ & 15 & 8 & 35 & 179 & $\operatorname{ker}\left(\mathcal{O}(15)^{43} \rightarrow \mathcal{O}(17)^{8}\right)$ & $509 / 35$ \\
\hline 129 & 15 & 9 & 15 & 0 & $\operatorname{coker}\left(\mathcal{O}(13)^{9} \rightarrow \mathcal{O}(14)^{24}\right)$ & $73 / 5$ \\
\hline 130 & 15 & 10 & 15 & 0 & $\operatorname{coker}\left(\mathcal{O}(13)^{10} \rightarrow \mathcal{O}(14)^{25}\right)$ & $44 / 3$ \\
\hline 131 & 15 & 11 & 15 & 0 & $\operatorname{coker}\left(\mathcal{O}(13)^{11} \rightarrow \mathcal{O}(14)^{26}\right)$ & $221 / 15$ \\
\hline 132 & 15 & 12 & 15 & 0 & $\operatorname{coker}\left(\mathcal{O}(13)^{12} \rightarrow \mathcal{O}(14)^{27}\right)$ & $74 / 5$ \\
\hline 133 & 15 & 13 & 15 & 0 & $\operatorname{coker}\left(\mathcal{O}(13)^{13} \rightarrow \mathcal{O}(14)^{28}\right)$ & $223 / 15$ \\
\hline 134 & 15 & 14 & 15 & 0 & $\operatorname{coker}\left(\mathcal{O}(13)^{14} \rightarrow \mathcal{O}(14)^{29}\right)$ & $224 / 15$ \\
\hline 135 & 15 & 15 & 15 & 0 & $\operatorname{coker}\left(\mathcal{O}(13)^{15} \rightarrow \mathcal{O}(14)^{30}\right)$ & 15 \\
\hline 136 & 16 & 0 & 16 & 0 & $\mathcal{O}(15)$ & 15 \\
\hline 137 & 16 & 1 & 18 & 0 & $\operatorname{ker}\left(\mathcal{O}(16)^{34} \rightarrow \mathcal{O}(17)^{16}\right)$ & $136 / 9$ \\
\hline 138 & 16 & 2 & 18 & 0 & $\operatorname{ker}\left(\mathcal{O}(16)^{33} \rightarrow \mathcal{O}(17)^{15}\right)$ & $91 / 6$ \\
\hline 139 & 16 & 3 & 18 & 0 & $\operatorname{ker}\left(\mathcal{O}(16)^{32} \rightarrow \mathcal{O}(17)^{14}\right)$ & $137 / 9$ \\
\hline 140 & 16 & 4 & 18 & 0 & $\operatorname{ker}\left(\mathcal{O}(16)^{31} \rightarrow \mathcal{O}(17)^{13}\right)$ & $275 / 18$ \\
\hline 141 & 16 & 5 & 18 & 0 & $\operatorname{ker}\left(\mathcal{O}(16)^{30} \rightarrow \mathcal{O}(17)^{12}\right)$ & $46 / 3$ \\
\hline 142 & 16 & 6 & $?$ & $?$ & $?$ & $?$ \\
\hline
\end{tabular}


continued from previous page

\begin{tabular}{|c|c|c|c|c|c|c|}
\hline$n$ & $r$ & $s$ & $\operatorname{deg} C_{n}$ & $\delta\left(C_{n}\right)$ & $E_{n}$ & $\mu\left(D_{n}\right)$ \\
\hline $143^{* *}$ & 16 & 7 & 31 & 98 & $\operatorname{coker}\left(\mathcal{O}(13)^{7} \rightarrow \mathcal{O}(15)^{38}\right)$ & $479 / 31$ \\
\hline 144 & 16 & 8 & 16 & 0 & $\operatorname{coker}\left(\mathcal{O}(14)^{8} \rightarrow \mathcal{O}(15)^{24}\right)$ & $31 / 2$ \\
\hline $145^{\ddagger}$ & 16 & 9 & 37 & 198 & $\operatorname{ker}\left(\mathcal{O}(16)^{45} \rightarrow \mathcal{O}(18)^{8}\right)$ & $576 / 37$ \\
\hline 146 & 16 & 10 & 16 & 0 & $\operatorname{coker}\left(\mathcal{O}(14)^{10} \rightarrow \mathcal{O}(15)^{26}\right)$ & $125 / 8$ \\
\hline 147 & 16 & 11 & 16 & 0 & $\operatorname{coker}\left(\mathcal{O}(14)^{11} \rightarrow \mathcal{O}(15)^{27}\right)$ & $251 / 16$ \\
\hline 148 & 16 & 12 & 16 & 0 & $\operatorname{coker}\left(\mathcal{O}(14)^{12} \rightarrow \mathcal{O}(15)^{28}\right)$ & $63 / 4$ \\
\hline 149 & 16 & 13 & 16 & 0 & $\operatorname{coker}\left(\mathcal{O}(14)^{13} \rightarrow \mathcal{O}(15)^{29}\right)$ & $253 / 16$ \\
\hline 150 & 16 & 14 & 16 & 0 & $\operatorname{coker}\left(\mathcal{O}(14)^{14} \rightarrow \mathcal{O}(15)^{30}\right)$ & $127 / 8$ \\
\hline 151 & 16 & 15 & 16 & 0 & $\operatorname{coker}\left(\mathcal{O}(14)^{15} \rightarrow \mathcal{O}(15)^{31}\right)$ & $255 / 16$ \\
\hline 152 & 16 & 16 & 16 & 0 & $\operatorname{coker}\left(\mathcal{O}(14)^{16} \rightarrow \mathcal{O}(15)^{32}\right)$ & 16 \\
\hline 153 & 17 & 0 & 17 & 0 & $\mathcal{O}(16)$ & 16 \\
\hline 154 & 17 & 1 & 19 & 0 & $\operatorname{ker}\left(\mathcal{O}(17)^{36} \rightarrow \mathcal{O}(18)^{17}\right)$ & $306 / 19$ \\
\hline 155 & 17 & 2 & 19 & 0 & $\operatorname{ker}\left(\mathcal{O}(17)^{35} \rightarrow \mathcal{O}(18)^{16}\right)$ & $307 / 19$ \\
\hline 156 & 17 & 3 & 19 & 0 & $\operatorname{ker}\left(\mathcal{O}(17)^{34} \rightarrow \mathcal{O}(18)^{15}\right)$ & $308 / 19$ \\
\hline 157 & 17 & 4 & 19 & 0 & $\operatorname{ker}\left(\mathcal{O}(17)^{33} \rightarrow \mathcal{O}(18)^{14}\right)$ & $309 / 19$ \\
\hline 158 & 17 & 5 & 19 & 0 & $\operatorname{ker}\left(\mathcal{O}(17)^{32} \rightarrow \mathcal{O}(18)^{13}\right)$ & $310 / 19$ \\
\hline 159 & 17 & 6 & 19 & 0 & $\operatorname{ker}\left(\mathcal{O}(17)^{31} \rightarrow \mathcal{O}(18)^{12}\right)$ & $311 / 19$ \\
\hline $160^{* *}$ & 17 & 7 & 33 & 113 & $\operatorname{coker}\left(\mathcal{O}(14)^{7} \rightarrow \mathcal{O}(16)^{40}\right)$ & $542 / 33$ \\
\hline $161^{* *}$ & 17 & 8 & 33 & 112 & $\operatorname{coker}\left(\mathcal{O}(14)^{8} \rightarrow \mathcal{O}(16)^{41}\right)$ & $544 / 33$ \\
\hline $162^{\ddagger}$ & 17 & 9 & 39 & 219 & $\operatorname{ker}\left(\mathcal{O}(17)^{48} \rightarrow \mathcal{O}(19)^{9}\right)$ & $215 / 13$ \\
\hline 163 & 17 & 10 & $?$ & $?$ & ? & $?$ \\
\hline 164 & 17 & 11 & 17 & 0 & $\operatorname{coker}\left(\mathcal{O}(15)^{11} \rightarrow \mathcal{O}(16)^{28}\right)$ & $283 / 17$ \\
\hline 165 & 17 & 12 & 17 & 0 & $\operatorname{coker}\left(\mathcal{O}(15)^{12} \rightarrow \mathcal{O}(16)^{29}\right)$ & $284 / 17$ \\
\hline 166 & 17 & 13 & 17 & 0 & $\operatorname{coker}\left(\mathcal{O}(15)^{13} \rightarrow \mathcal{O}(16)^{30}\right)$ & $285 / 17$ \\
\hline 167 & 17 & 14 & 17 & 0 & $\operatorname{coker}\left(\mathcal{O}(15)^{14} \rightarrow \mathcal{O}(16)^{31}\right)$ & $286 / 17$ \\
\hline 168 & 17 & 15 & 17 & 0 & $\operatorname{coker}\left(\mathcal{O}(15)^{15} \rightarrow \mathcal{O}(16)^{32}\right)$ & $287 / 17$ \\
\hline 169 & 17 & 16 & 17 & 0 & $\operatorname{coker}\left(\mathcal{O}(15)^{16} \rightarrow \mathcal{O}(16)^{33}\right)$ & $288 / 17$ \\
\hline 170 & 17 & 17 & 17 & 0 & $\operatorname{coker}\left(\mathcal{O}(15)^{17} \rightarrow \mathcal{O}(16)^{34}\right)$ & 17 \\
\hline 171 & 18 & 0 & 18 & 0 & $\mathcal{O}(17)$ & 17 \\
\hline 172 & 18 & 1 & 20 & 0 & $\operatorname{ker}\left(\mathcal{O}(18)^{38} \rightarrow \mathcal{O}(19)^{18}\right)$ & $171 / 10$ \\
\hline 173 & 18 & 2 & 20 & 0 & $\operatorname{ker}\left(\mathcal{O}(18)^{37} \rightarrow \mathcal{O}(19)^{17}\right)$ & $343 / 20$ \\
\hline 174 & 18 & 3 & 20 & 0 & $\operatorname{ker}\left(\mathcal{O}(18)^{36} \rightarrow \mathcal{O}(19)^{16}\right)$ & $86 / 5$ \\
\hline 175 & 18 & 4 & 20 & 0 & $\operatorname{ker}\left(\mathcal{O}(18)^{35} \rightarrow \mathcal{O}(19)^{15}\right)$ & $69 / 4$ \\
\hline 176 & 18 & 5 & 20 & 0 & $\operatorname{ker}\left(\mathcal{O}(18)^{34} \rightarrow \mathcal{O}(19)^{14}\right)$ & $173 / 10$ \\
\hline 177 & 18 & 6 & 20 & 0 & $\operatorname{ker}\left(\mathcal{O}(18)^{33} \rightarrow \mathcal{O}(19)^{13}\right)$ & $347 / 20$ \\
\hline 178 & 18 & 7 & 20 & 0 & $\operatorname{ker}\left(\mathcal{O}(18)^{32} \rightarrow \mathcal{O}(19)^{12}\right)$ & $87 / 5$ \\
\hline $179^{* *}$ & 18 & 8 & 35 & 128 & $\operatorname{coker}\left(\mathcal{O}(15)^{8} \rightarrow \mathcal{O}(17)^{43}\right)$ & $611 / 35$ \\
\hline 180 & 18 & 9 & 18 & 0 & $\operatorname{coker}\left(\mathcal{O}(16)^{9} \rightarrow \mathcal{O}(17)^{27}\right)$ & $35 / 2$ \\
\hline $181^{\ddagger}$ & 18 & 10 & 41 & 240 & $\operatorname{ker}\left(\mathcal{O}(18)^{50} \rightarrow \mathcal{O}(20)^{9}\right)$ & $720 / 41$ \\
\hline
\end{tabular}


continued from previous page

\begin{tabular}{ccccccc}
\hline$n$ & $r$ & $s$ & $\operatorname{deg} C_{n}$ & $\delta\left(C_{n}\right)$ & $E_{n}$ & $\mu\left(D_{n}\right)$ \\
\hline 182 & 18 & 11 & $?$ & $?$ & $?$ & $?$ \\
183 & 18 & 12 & 18 & 0 & $\operatorname{coker}\left(\mathcal{O}(16)^{12} \rightarrow \mathcal{O}(17)^{30}\right)$ & $53 / 3$ \\
184 & 18 & 13 & 18 & 0 & $\operatorname{coker}\left(\mathcal{O}(16)^{13} \rightarrow \mathcal{O}(17)^{31}\right)$ & $319 / 18$ \\
185 & 18 & 14 & 18 & 0 & $\operatorname{coker}\left(\mathcal{O}(16)^{14} \rightarrow \mathcal{O}(17)^{32}\right)$ & $160 / 9$ \\
186 & 18 & 15 & 18 & 0 & $\operatorname{coker}\left(\mathcal{O}(16)^{15} \rightarrow \mathcal{O}(17)^{33}\right)$ & $107 / 6$ \\
187 & 18 & 16 & 18 & 0 & $\operatorname{coker}\left(\mathcal{O}(16)^{16} \rightarrow \mathcal{O}(17)^{34}\right)$ & $161 / 9$ \\
188 & 18 & 17 & 18 & 0 & $\operatorname{coker}\left(\mathcal{O}(16)^{17} \rightarrow \mathcal{O}(17)^{35}\right)$ & $323 / 18$ \\
189 & 18 & 18 & 18 & 0 & $\operatorname{coker}\left(\mathcal{O}(16)^{18} \rightarrow \mathcal{O}(17)^{36}\right)$ & 18 \\
\hline
\end{tabular}




\section{Bibliography}

[1] Daniele Arcara, Aaron Bertram, Izzet Coskun, and Jack Huizenga. The minimial model program for the Hilbert scheme of points on $\mathbb{P}^{2}$ and Bridgeland stability. Preprint.

[2] E. Arbarello, M. Cornalba, P. A. Griffiths, and J. Harris. Geometry of algebraic curves. Vol. I, volume 267 of Grundlehren der Mathematischen Wissenschaften [Fundamental Principles of Mathematical Sciences]. Springer-Verlag, New York, 1985.

[3] Guntram Bohnhorst and Heinz Spindler. The stability of certain vector bundles on $\mathbf{P}^{n}$. Complex algebraic varieties (Bayreuth, 1990), volume 1507 of Lecture Notes in Math. Springer, Berlin, 1992.

[4] Maria Chiara Brambilla. Simplicity of generic Steiner bundles. Boll. Unione Mat. Ital. Sez. B Artic. Ric. Mat. (8), 8(3):723-735, 2005.

[5] Maria Chiara Brambilla. Cokernel bundles and Fibonacci bundles. Math. Nachr., 281(4):499-516, 2008.

[6] J.-M. Drezet and J. Le Potier. Fibrés stables et fibrés exceptionnels sur $\mathbf{P}_{2}$. Ann. Sci. École Norm. Sup. (4), 18(2):193-244, 1985.

[7] David Eisenbud. The geometry of syzygies, volume 229 of Graduate Texts in Mathematics. Springer-Verlag, New York, 2005.

[8] David Eisenbud, Mark Green, and Joe Harris. Cayley-Bacharach theorems and conjectures. Bull. Amer. Math. Soc. (N.S.), 33(3):295-324, 1996.

[9] Gerd Faltings. Stable G-bundles and projective connections. J. Algebraic Geom., 2(3):507-568, 1993.

[10] J. Fogarty. Algebraic families on an algebraic surface. Amer. J. Math., 90(2):511-521, 1968.

[11] J. Fogarty. Algebraic families on an algebraic surface, II, the Picard scheme of the punctual Hilbert scheme. Amer. J. Math., 95(3):660-687, 1973.

[12] Hubert Flenner. Restrictions of semistable bundles on projective varieties. Comment. Math. Helv., 59(4):635-650, 1984. 
[13] Robin Hartshorne. Algebraic geometry, volume 52 of Graduate Texts in Mathematics. Springer-Verlag, New York, 1977.

[14] James McKernan. Mori dream spaces. Japan. J. Math., 5(1):127-151, 2010.

[15] V. B. Mehta and A. Ramanathan. Semistable sheaves on projective varieties and their restriction to curves. Math. Ann., 258(3):213-224, 1981/82.

[16] Christian Okonek, Michael Schneider, and Heinz Spindler. Vector bundles on complex projective spaces, volume 3 of Progress in Mathematics. Birkhäuser Boston, Mass., 1980.

[17] E. A. Tevelev. Projective duality and homogeneous spaces, volume 133 of Encyclopaedia of Mathematical Sciences. Springer-Verlag, New York, 2005. 